\title{
APPLICATION OF SUPERCRITICAL FLUID EXTRACTION FOR THE SEPARATION OF NUTRACEUTICALS AND OTHER PHYTOCHEMICALS FROM PLANT MATERIAL
}

\author{
Miha Oman, Mojca Škerget, Željko Knez \\ Faculty of Chemistry and Chemical Engineering, \\ University of Maribor, Smetanova 17, \\ SI-2000 Maribor, Slovenia \\ zeljko.knez@um.si
}

\begin{abstract}
In the present work, a literature review of the application of supercritical fluid extraction (SFE) for the isolation of nutraceuticals and some other phytochemicals up to December of 2012 is presented. The manuscript provides knowledge of SFE processes and possible applications of SFE for the extraction of bioactive compounds that serve as nutraceuticals. Compounds are classified into groups based on their chemical nature (carotenoids, flavonoids and other phenolic compounds, essential oils, lipids and fatty acids, and alkaloids and other bioactive phytochemicals), and they are reviewed in tabular form along with the plant material from which they were extracted using supercritical fluids.
\end{abstract}

Keywords: supercritical fluid extraction; nutraceuticals; phytochemicals; plant material

\section{ПРИМЕНА НА СУПЕРКРИТИЧНАТА ФЛУИДНА ЕКСТРАКЦИЈА ЗА СЕПАРАЦИЈА НА НУТРИЕНТИ И ДРУГИ ФИТОХЕМИКАЛИИ ОД РАСТИТЕЛЕН МАТЕРИЈАЛ}

Во овој труд е презентиран прегледот на литературата објавена до декември 2012 година, а се однесува на извлекување на нутриенти и некои фитохемикалии со суперкритичната флуидна екстракција (SFE). Презентирани се сознанијата за процесите на суперкритичната флуидна екстракција како можност за извлекување на биоактивни компоненти кои се користат како нутриенти. Соединенијата се класифицирани во групи врз основа на нивната хемиска природа (каротеноиди, флавоноиди и други фенолни соединенија, есенцијални масла, липиди, масни киселини, алкалоиди и други биоактивни фитохемикалии). Во трудов тие се презентирани во табели заедно со растителен материјал од кој се извлекувани со користење суперкритична флуидна екстракција.

Клучни зборови: суперкритична флуидна екстракција; нутриенти; фитохемикалии; растителен материјал 


\section{INTRODUCTION}

In recent time, there is greater emphasis on the recovery of high value-added products by using sustainable technologies. One of the ways to achieve this is the application of sub- and supercritical fluids $(\mathrm{ScF})$. ScF can be applied as solvents for precipitation and micronization (PGSS ${ }$, RESS, etc.), as reaction medium, as mobile phase for chromatography (supercritical fluid chromatography - SFC), and as solvent for extraction, which is the most investigated process. From an economic point of view, technologies involving elevated pressures require high investment costs for high-pressure equipment. Because of this, it is reasonable to apply supercritical fluid extraction (SFE) for the separation of components with high added value, such as nutraceuticals, pharmaceuticals, food additives, or components with high feed-to-solvent $(\mathrm{F} / \mathrm{S})$ extraction ratio.

SFE is a separation process where solid or liquid matter is processed with $\mathrm{ScF}$ in order to obtain soluble compounds from mixtures. ScF offers a variety of applications due to specific properties, which can be relatively easily adjusted with changing pressure and temperature. A fluid above critical temperature has gaslike viscosity, liquidlike density, and its diffusion magnitude of order is between the two fluid states.

An important factor that has to be considered is the mass transfer of the solute in the supercritical solvent. Mass transfer depends on the solubility of the solute in the given solvent. Different compounds have different solubilities at various operating conditions. In general, temperature and pressure have the biggest influence on the solubility of compounds in supercritical fluids. Temperature has two competing effects on solubility. First, increasing the temperature at constant pressure decreases the density of the solvent. Thus, solubility of the solute is decreased. On the other hand, by increasing the temperature at constant density, the vapor pressure of the solute is increased. Therefore a solute is more soluble in a supercritical fluid. Which effect will prevail is dependent on the properties of the system.

The effect of pressure is more direct. With increasing the pressure of a supercritical medium, higher densities are achieved; the higher the density of the medium, the higher the solubility of the solute [1]. The most common solvent used as a supercritical fluid is carbon dioxide $\left(\mathrm{CO}_{2}\right)$. When polar components are extracted and supercritical $\mathrm{CO}_{2}\left(\mathrm{SC}-\mathrm{CO}_{2}\right)$ is used as a solvent, a polar modifier or co-solvent is mixed with $\mathrm{SC}-\mathrm{CO}_{2}$ to enhance solubility. Examples of such a modifier are methanol, ethanol, etc.

When a modifier is added, not only solubility but also viscosity and density are increased. Because of the increase in density and viscosity, the diffusivity in the mobile phase decreases, hence mass transfer is reduced.

To obtain the highest yields possible, the process has to be optimized. First, the influence of process parameters on the extraction has to be studied. Regarding the results, the optimal operating parameters are chosen. One way to determine the optimal parameters is with the use of response surface methodology (RSM). This method uses the multiple regression model (a polynomial second-order equation), from which optimum parameters are selected. This model is described as well in the work of Wang et al. [2], where optimization with RSM for SFE of essential oils from Cyperus rotundus is described.

Last but not the least, the extraction rates depend on the morphology of the material and the location of the solute in plant material. If the desired solute is on the surface of the material, generally extraction rates are high. However, when desired compounds are deeper in the material, it takes more time to extract them. In those cases, mass transfer depends on particle shape, size, and the porosity of the solid material. If the structure of the material is more complex and the desired compounds are deeper inside, a greater resistance for diffusion is expected [3-7]. Therefore, the preparation of a sample is very important for SFE of natural matter $[8,9]$. Usually, a material has to be mechanically pretreated - i.e. mechanically processed by grinding, milling, cutting, etc. - to reduce mean particle size. As mentioned in this section, smaller particles provide faster extraction due to lower diffusion paths and less diffusion resistance. The importance of proper material pre- 
treatment is presented in the work of Uquiche et al. [10], where kinetics of SFE of pretreated boldo was studied.

The aim of this work is to review investigations of the separation of compounds from natural matter with SFE performed in recent years. Several reviews have been published before. Among them were the reviews of Reverchon and De Marco [7], Pereira and Meireles [6], and Sovova and Stateva [11]. Hence there is no reason for repetition. The present work is focused on extractions of nutraceuticals and other phytochemicals from plant material and the collection of investigations in tabular form. Sections of this paper are divided into bigger groups (carotenoids, flavonoids and phenolic compounds, essential oils, lipid and fatty acids, and alkaloids and other phytochemicals). Compounds that belong to a certain group are listed in tabular form in the corresponding section. Key words used in the present work for searching the literature are listed in Table 1.

Ta b le 1

Keywords used for searching the literature

\begin{tabular}{|c|c|c|}
\hline Group & Subgroup & Keywords \\
\hline \multirow[t]{6}{*}{ Nutraceuticals } & General & $\begin{array}{l}\text { nutraceuticals, } \\
\text { supercritical fluid } \\
\text { extraction }\end{array}$ \\
\hline & Carotenoids & $\begin{array}{l}\text { carotenoids, } \\
\text { supercritical fluid } \\
\text { extraction }\end{array}$ \\
\hline & Flavonoids & $\begin{array}{l}\text { flavonoids, } \\
\text { supercritical fluid } \\
\text { extraction }\end{array}$ \\
\hline & $\begin{array}{l}\text { Phenolic } \\
\text { compounds }\end{array}$ & $\begin{array}{l}\text { phenolic, phenols, } \\
\text { supercritical fluid } \\
\text { extraction }\end{array}$ \\
\hline & Essential oils & $\begin{array}{l}\text { essential oils, } \\
\text { supercritical fluid } \\
\text { extraction }\end{array}$ \\
\hline & Lipids, FFA & $\begin{array}{l}\text { lipids, FFA, } \\
\text { supercritical fluid } \\
\text { extraction }\end{array}$ \\
\hline
\end{tabular}

\section{SFE OF NATURAL MATTER: NUTRACEUTICALS}

Nutraceuticals are substances that may be considered as food or part of a food and provide medical or health benefits, including the prevention and treatment of diseases [12]. Such products may range from isolated nutrients, dietary supplements and diets to genetically engineered "designer" food, herbal products, and processed food (such as cereals, soups, and beverages) [12].

In some aspects, functional food is related to nutraceuticals. Functional food provides health benefits over normal nutrition, and it is different from medical food or dietary supplements [12].

The nutraceuticals reviewed in this work are based on their chemical nature, classified into the following groups: carotenoids, flavonoids and other phenolic compounds, fatty acids and lipids, essential oils, and alkaloids and other phytochemicals.

\subsection{Carotenoids}

Carotenoids are divided into two subgroups: carotenes and oxygenized hydrocarbons xanthophylls. They consist of eight isoprenoid units joined in such a manner that their arrangement is reversed at the center of the molecule. All carotenoids may be formally derived from the acyclic $\mathrm{C}_{40} \mathrm{H}_{56}$ structure [13]. Trivial names are usually used for common carotenoids. The functional groups most frequently observed in the group of xanthophylls are hydroxy, methoxy, oxo, carboxy, and epoxy [13].

Carotenoids in food are recognized as antioxidants and pigments [14, 15]. As antioxidants, they affect human living tissues by preventing the oxidation of the molecules, inhibiting harmful microbiological activities, and protecting against cancer [16]. In Table 2 , investigations of SFE of carotenoids from different plant material are listed. 
Tab 1 e 2

Application of SFE for the extraction of carotenoids from plants

\begin{tabular}{|c|c|c|c|}
\hline Compound(s) & Plant material (Biological name) & Methods/solvents & Reference(s) \\
\hline Astaxanthin & $\begin{array}{l}\text { Microalgea (Haematococcus } \\
\text { pluvialis) }\end{array}$ & $\mathrm{SFE} / \mathrm{CO}_{2}+$ ethanol & [23-26] \\
\hline Astaxanthin & Microalgea (Chlorella vulgaris) & $\mathrm{SFE} / \mathrm{CO}_{2}$ & [27] \\
\hline Carotenes & $\begin{array}{l}\text { Seabuckthorn (Hippophae rhamnoids } \\
\text { L.) }\end{array}$ & $\mathrm{SFE} / \mathrm{CO}_{2}$ & {$[28,29]$} \\
\hline Canthaxanthin & Microalgea (Chlorella vulgaris) & $\mathrm{SFE} / \mathrm{CO}_{2}$ & {$[27]$} \\
\hline Fucoxanthin & Microalgea (Undaria pinnatifida) & $\mathrm{SFE} / \mathrm{CO}_{2}+$ ethanol & {$[30]$} \\
\hline Lutein & Daylily (Hemerocallis disticha) & $\mathrm{SFE} / \mathrm{CO}_{2}$ & {$[31]$} \\
\hline Lutein & Marigold (Tagetes erecta) & $\mathrm{SFE} / \mathrm{CO}_{2}$ & {$[32-34]$} \\
\hline Lutein & Spearmint (Mentha spicata) & $\mathrm{SC}-\mathrm{CO}_{2}$ & {$[35]$} \\
\hline Lutein & Pumpkin (Curcurbita moschata) & $\mathrm{SE}, \mathrm{SFE} / \mathrm{CO}_{2}$ & {$[36,37]$} \\
\hline Lutein & Stinging nettle (Urtica dioica) & $\mathrm{SC}-\mathrm{CO}_{2}+$ ethanol & {$[38]$} \\
\hline Lutein & Microalgea (Chlorella vulgaris) & $\mathrm{SFE} / \mathrm{CO}_{2}$ & {$[27,39]$} \\
\hline Lycopene & Fungal species (Blakeslea trispora) & $\mathrm{SFE} / \mathrm{CO}_{2}$ & [40] \\
\hline Lycopene & Hazelnut (Corylus avellana L.) & $\mathrm{SFE} / \mathrm{CO}_{2}$ & [41] \\
\hline Lycopene & Tomato (Lycopersicum esculentum L.) & $\mathrm{SFE} / \mathrm{CO}_{2}+$ ethanol, water, canola oil & {$[42,43]$} \\
\hline Lycopene & Tomato (Lycopersicum esculentum L.) & $\mathrm{SFE} / \mathrm{CO}_{2}$ & {$[41,44]$} \\
\hline Lycopene & Tomato (Lycopersicum esculentum L.) & $\mathrm{SFE} / \mathrm{CO}_{2}$ & {$[45,46]$} \\
\hline Lycopene & Tomato (Lycopersicum esculentum L.) & $\mathrm{SE} /$ acetone/water, $\mathrm{SFE} / \mathrm{CO}_{2}$ & {$[16,47-49]$} \\
\hline Lycopene & Guava (Psidium guajava) & $\mathrm{SFE} / \mathrm{CO}_{2}$ & {$[50]$} \\
\hline Lycopene & $\begin{array}{l}\text { Brazilian cherry (Hymenaea } \\
\text { courbaril) }\end{array}$ & $\mathrm{SFE} / \mathrm{CO}_{2}$ & {$[51]$} \\
\hline Lycopene & Pumpkin (Curcurbita moschata) & $\mathrm{SE}, \mathrm{SFE} / \mathrm{CO}_{2}$ & {$[36,37]$} \\
\hline N.D. & Microalgea (Chlorococcum littorale) & $\mathrm{SFE} / \mathrm{CO}_{2}$ & {$[52]$} \\
\hline N.D. & Dog rose (Rosa canina) & $\mathrm{SFE} / \mathrm{CO}_{2}+$ ethanol & {$[53]$} \\
\hline N.D. & lotus (Nelumbo nucifera Gaertn) & $\mathrm{SFE} / \mathrm{CO}_{2}$ & [54] \\
\hline N.D. & Persimmon peels (Japan) & $\mathrm{SFE} / \mathrm{CO}_{2}+$ ethanol & {$[55]$} \\
\hline Xanthophylls & Paprika (Capsicum аппиит) & $\mathrm{SFE} / \mathrm{CO}_{2}$ & {$[56]$} \\
\hline Zeaxanthin & Daylily (Hemerocallis disticha) & $\mathrm{SFE} / \mathrm{CO}_{2}$ & {$[31]$} \\
\hline Zeaxanthin & Microalgea (Spirulina Pacifica) & $\mathrm{SFE} / \mathrm{CO}_{2}+$ ethanol & {$[57]$} \\
\hline Zeaxanthin & $\begin{array}{l}\text { Microalgea (Nannochloropsis } \\
\text { oculata) }\end{array}$ & $\mathrm{SFE} / \mathrm{CO}_{2}$ & {$[58,59]$} \\
\hline Zeaxanthin & Microalgea (Spirulina platensis) & $\mathrm{SFE} / \mathrm{CO}_{2}+$ ethanol & {$[60]$} \\
\hline Zeaxanthin & $\begin{array}{l}\text { Microalgea (Paracoccus } \\
\text { zeaxanthinifaciens) }\end{array}$ & $\mathrm{SFE} / \mathrm{CO}_{2}+$ ethanol & {$[61]$} \\
\hline$\beta$-carotene & Paprika (Capsicum аппиит) & $\mathrm{SFE} / \mathrm{CO}_{2}$ & {$[56]$} \\
\hline$\beta$-carotene & Red pepper (Capsicum annum L.) & $\mathrm{SFE} / \mathrm{CO}_{2}$ & {$[62]$} \\
\hline$\beta$-carotene & Palm oil (Elaeis guineensis) & $\mathrm{SFE} / \mathrm{CO}_{2}$ & {$[63]$} \\
\hline$\beta$-carotene & Fungal species (Blakeslea trispora) & $\mathrm{SFE} / \mathrm{CO}_{2}$ & {$[40]$} \\
\hline$\beta$-carotene & Tomato (Lycopersicum esculentum L.) & $\mathrm{SFE} / \mathrm{CO}_{2}$ & {$[45,46]$} \\
\hline$\beta$-carotene & Microalgea (Dunaliella bardawil) & $\mathrm{SFE} / \mathrm{CO}_{2}$ & [19] \\
\hline
\end{tabular}




$\begin{array}{llll}\beta \text {-carotene } & \text { Spearmint (Mentha spicata) } & \mathrm{SFE} / \mathrm{CO}_{2} & {[35]} \\ \beta \text {-carotene } & \text { Tomato (Lycopersicum esculentum L.) } & \mathrm{SFE} / \mathrm{CO}_{2} & {[16]} \\ \beta \text {-carotene } & \text { Lotus (Nelumbo nucifera) } & \mathrm{SFE} / \mathrm{CO}_{2} & {[64]} \\ \beta \text {-carotene } & \begin{array}{l}\text { Brazilian cherry } \text { (Hymenaea } \\ \text { courbaril) }\end{array} & \mathrm{SFE} / \mathrm{CO}_{2} & {[51]} \\ \beta \text {-carotene } & \text { Microalgea (Chlorella vulgaris) } & \mathrm{SFE} / \mathrm{CO}_{2} & {[27]} \\ \beta \text {-carotene } & \text { Microalgea (Dunaliella salina) } & \mathrm{SFE} / \mathrm{CO}_{2} & {[27]} \\ \beta \text {-carotene } & \text { Microalgea (Spirulina platensis) } & \mathrm{SFE} / \mathrm{CO}_{2}+\text { ethanol } & {[60]} \\ \beta \text {-carotene } & \text { Apricot (Prunus armeniaca) } & \mathrm{SFE} / \mathrm{CO}_{2} & {[65]} \\ \beta \text {-carotene } & \text { Carrot (Daucus carota) } & \mathrm{SFE} / \mathrm{CO}_{2} & {[66]} \\ \beta \text {-carotene } & \text { Paprika (Capsicum annuum) } & \mathrm{SFE} / \mathrm{CO}_{2} ;+\mathrm{DMP} ;+\mathrm{DEP} ;+\mathrm{TEOF} & {[8]} \\ \beta \text {-cryptoxanthin } & \text { Microalgea (Spirulina pacifica) } & \mathrm{SFE} / \mathrm{CO}_{2}+\text { ethanol } & {[57]} \\ \beta \text {-carotene } & \text { Palm oil (Elaeis guineensis) } & \mathrm{SFE} / \mathrm{R} 134 a^{2} & {[67]}\end{array}$

\subsubsection{Carotenes}

Palozza and Krinsky [17] reviewed carotenes and the biological antioxidant properties of $\beta$-carotene. The work of Palozza and Krinsky [17] also describes SFE and the influence of pressure and temperature on carotene extraction. It was concluded in most cases that by increasing pressure, hence increasing density, the solubility of carotenes in $\mathrm{CO}_{2}$ generally also increases; therefore, higher extraction yields are achieved in less time at moderate temperatures. A bigger amount of extracted carotenes means bigger antioxidant potential. If extraction is carried out at a temperature that is too high, $\beta$-carotene could be decomposed; therefore less antioxidant potential is observed.

The extraction of $\beta$-carotene from material that contains too much water is less effective [8]. The material should be dried to achieve better extraction efficiency. Weathers et al. [8] suggested the use of freeze-drying in order to preserve thermolabile compounds.

Subra et al. [18] examined the influence of particle size and the influence of $\mathrm{CO}_{2}$ and/or $\mathrm{N}_{2} \mathrm{O}$ and their flow rates on the extraction of $\beta$-carotene from freeze-dried carrots. Subra et al. [18] observed that with smaller particles, higher yields were obtained, due to grinding, which destroyed the inner structures of particles. Furthermore, with higher solvent flow rates, higher extraction rates were achieved, due to an increased external mass transfer. Extractions carried out with $\mathrm{N}_{2} \mathrm{O}$ were slightly faster than with $\mathrm{CO}_{2}$ because of the higher solvent power of $\mathrm{N}_{2} \mathrm{O}$.

Gamlieli-Bonshtein et al. [19] investigated SFE for the separation of trans-cis isomers of $\beta$-carotene from concentrates containing several carotenoids. They concluded that SFE can be used to separate geometrical isomers of $\beta$-carotene based on their different solubilities in $\mathrm{SC}-\mathrm{CO}_{2}$. According to their investigations, it could be concluded that for SFE, an excessive research of solubility data is often required.

Baysal et al. [15] studied the extraction of $\beta$-carotene and lycopene from tomato wastes with $\mathrm{CO}_{2}$. They investigated the influence of operating parameters (pressure, temperature, flow rate, extraction time, and co-solvent addition) on extraction yields. At the highest temperature, with a pressure of $30 \mathrm{MPa}$, yield was the highest; however, lycopene could not be totally recovered because of partial thermal degradation. In the presence of a co-solvent, the recovery of extracted compounds increased, because solubility increased [15]. A similar research was done by Doker et al. [20]. The modeling they performed [20] show that external and internal mass-transfer resistances are very important and control overall extraction rates. 
Pressure is one of the most important parameters influencing the extraction and concentration of carotenes. By changing the pressure, the solubility of carotenes in $\mathrm{SC}-\mathrm{CO}_{2}$ is changed. When extraction is pressure-programmed, which means that pressure automatically or manually increases after a period of time, separate fractions of compounds can be collected. Lau et al. [21] investigated the pressure-programmed extraction of palm-pressed mesocarp fiber, where pressure was subsequently increased, first from $10 \mathrm{MPa}$ to 20 $\mathrm{MPa}$ after the initial $3 \mathrm{~h}$, then from $20 \mathrm{MPa}$ to 30 $\mathrm{MPa}$ after $1 \mathrm{~h}$, where it was maintained for $6 \mathrm{~h}$. $\beta$-carotene (provitamin of vitamin $\mathrm{E}$ ) and squalene were extracted at lower pressures $(10 \mathrm{MPa})$ of $\mathrm{SC}-\mathrm{CO}_{2}$; therefore these compounds were collected mainly in the early stage, while other carotenes were collected in the following fractions at pressures above $20 \mathrm{MPa}$. Often co-solvents such as methanol, ethanol, or acetone are used to enhance solubility, but in some cases edible oils as co-solvents are used for the same purpose [22].

In the work of Ciurlia et al. [41], an innovative separation method is presented, where dried tomato and roasted hazelnuts are mixed. Oil extracted from hazelnut helps with the extraction of carotenes, acting as an actual co-solvent. This method is interesting because it requires less addition of other co-solvents.

Sanal et al. [65] investigated parameters that might influence extraction yields besides those previously mentioned in this section. The influence of drying procedure, sample amount, and influence of DMP could be obtained from their work [65].

In order to calculate extraction yields, extracts were characterized with several analytical methods, such as high-performance liquid chromatography (HPLC) and spectrophotometry in the UV/VIS region. In the literature [68-70], some other analytical methods are described.

\subsubsection{Xanthophylls}

Xanthophylls are a group of naturally occurring oxygenized carotenoids produced mainly by plants and microorganisms.
They are applied as food additives and food colorants for antioxidant purposes and cancer prevention. Lutein, zeaxanthin, and cryptoxanthin are major xanthophyll carotenoids in the human plasma. Besides those, there are several other important xanthophylls, such as canthaxanthin, astaxanthin, neoxanthin, and violaxanthin [71].

Lutein and zeaxanthin are both present in the cells of the human eye [72]. Lutein not only serves as a pigment or colorant but also prevents diseases, such as ophthalmopathy [55]. Takahashi et al. [55] carried out the extraction of xanthophylls and other carotenoids from the Japanese persimmon with the addition of ethanol as modifier. It was observed that a higher concentration of the added modifier decreases the selectivity of the solvent for carotenoids, hence other compounds other than carotenoids are extracted. Like modifiers, mostly organic solvents are used, but vegetable oils can modify the mobile phase as well. Gao et al. [33] investigated the extraction of lutein from the marigold flower with $\mathrm{CO}_{2}$ in the presence of vegetable oils as modifier. Results showed that the addition of edible oils to the mobile phase results to a higher amount of lutein extracted. Other sources of lutein are listed in Table 2 along with other carotenoids extracted from plants. Wu et al. [73] investigated SFE of lutein from cultivated Chlorella pyrenoidosa, and they observed that this species contain an amount of lutein comparable to that found in the marigold flower; hence this culture could be used as an alternative.

Among other xanthophylls, astaxanthin provides health benefits also. It contains two oxygenated groups on each ring structure, which are responsible for its enhanced antioxidant properties [74]. Astaxanthin can be obtained by SFE from pigments of $H$. pluvialis [24] and some other microalgae or sea fruits [27, 75-79].

\subsection{Flavonoids and other phenolic compounds}

Applications of SFE for the extraction of flavonoids and other phenolic compounds from plant material are presented in Table 3. 
Table 3

Application of SFE for separation of phenolic compounds from plants

\begin{tabular}{|c|c|c|c|}
\hline Compound(s) & $\begin{array}{l}\text { Plant material } \\
\text { (Biological name) }\end{array}$ & Methods/solvents & Reference(s) \\
\hline Anthocyanins & Raspberry (Rubus idaeus) & $\mathrm{SFE} / \mathrm{CO}_{2}$ & [109] \\
\hline Anthocyanins & $\begin{array}{l}\text { Blueberry (Vaccinium } \\
\text { corymbosum) }\end{array}$ & $\mathrm{SFE} / \mathrm{CO}_{2}$ & [109] \\
\hline Anthocyanins & $\begin{array}{l}\text { Cranberry (Vaccinium } \\
\text { oxycoccos) }\end{array}$ & $\mathrm{SFE} / \mathrm{CO}_{2}$ & [109] \\
\hline Anthocyanins & Elderberry (Sambucus nigra L.) & $\mathrm{SFE} / \mathrm{CO}_{2}$ & [110] \\
\hline Apigenin & $\begin{array}{l}\text { Chamomile (Chamomilla } \\
\text { recutita) }\end{array}$ & $\mathrm{SFE} / \mathrm{CO}_{2}+$ ethanol & {$[94,101,102,111]$} \\
\hline Apigenin & Olive (Olea europaea) & $\mathrm{SFE} / \mathrm{CO}_{2}$ & [112] \\
\hline Apigenin & Strobilanthes crispus & $\mathrm{SFE} / \mathrm{CO}_{2}+$ ethanol & [113] \\
\hline Apigenin & Marchantia convoluta & $\mathrm{SFE} / \mathrm{CO}_{2}+$ ethanol & {$[92,114-116]$} \\
\hline Apigenin & Shiyacha (Adinandra nitida) & $\mathrm{SFE} / \mathrm{CO}_{2} ;$ methanol & [117] \\
\hline Apigenin & Spearmint (Mentha spicata L.) & $\mathrm{SFE} / \mathrm{CO}_{2}$ & {$[118,119]$} \\
\hline Artepillin C & Brazilian propolis & $\mathrm{SFE} / \mathrm{CO}_{2}$ & [120] \\
\hline Artepillin C & Brazilian propolis & $\begin{array}{l}\mathrm{SFE} ; \mathrm{SE} / \mathrm{CO}_{2}+\text { ethyl acetate; } \\
\text { ethyl acetate }\end{array}$ & {$[121,122]$} \\
\hline Artepillin C & Brazilian propolis & $\begin{array}{l}\mathrm{SFE} ; \mathrm{SE} / \mathrm{CO}_{2}+\text { ethanol; ethanol, } \\
\text { ethyl acetate, } \\
\text { chloroform, } n \text {-hexane, water, } \\
\text { water + ethanol }\end{array}$ & [123] \\
\hline Baicalin & $\begin{array}{l}\text { Baikal skullcap (Scutellaria } \\
\text { baicalensis) }\end{array}$ & $\begin{array}{l}\mathrm{SFE} / \mathrm{CO}_{2}+\text { methanol, + ethanol, }+ \\
\text { 1,2-propanediol }\end{array}$ & [98] \\
\hline Baicalin & $\begin{array}{l}\text { Blue skullcap (Scutellaria } \\
\text { lateriflora) }\end{array}$ & $\mathrm{SFE} / \mathrm{CO}_{2}+$ ethanol, $\mathrm{SHW}$ & [97] \\
\hline Baicalin & Skullcap (Scutellariae radix) & $\mathrm{SE} ; \mathrm{SFE} / \mathrm{CO}_{2}+$ methanol & [124] \\
\hline Baicelein & Skullcap (Scutellariae radix) & $\mathrm{SE} ; \mathrm{SFE} / \mathrm{CO}_{2}+$ methanol & [124] \\
\hline Camellianin A & Shiyacha (Adinandra nitida) & $\mathrm{SFE} / \mathrm{CO}_{2} ;$ methanol & [117] \\
\hline Camellianin A & $\begin{array}{l}\text { Macela (Achyrocline } \\
\text { satureioides) }\end{array}$ & $\mathrm{SFE} / \mathrm{CO}_{2}+$ ethanol & [125] \\
\hline Catechin & Tea (Camellia sinensis) & $\mathrm{SFE} / \mathrm{CO}_{2}$ & [126] \\
\hline Catechin & Rooibos (Aspalathus linearis) & $\mathrm{SFE} / \mathrm{CO}_{2}$ & [126] \\
\hline Catechin & Tea (Camellia sinensis) & $\mathrm{SFE} / \mathrm{CO}_{2}+$ ethanol & [127] \\
\hline Catechin & Grape & $\begin{array}{l}\mathrm{SE} ; \mathrm{SFE} / \text { ethanol + water; } \mathrm{CO}_{2},+ \\
\text { ethanol }\end{array}$ & $\begin{array}{l}{[83,84,87,92,93,128-} \\
130]\end{array}$ \\
\hline Catechin & Spearmint (Mentha spicata L.) & $\mathrm{SFE} / \mathrm{CO}_{2}$ & {$[118,119]$} \\
\hline Catechin & Olive (Olea europaea) & $\mathrm{SE} ; \mathrm{SFE} /$ ethanol; $\mathrm{CO}_{2}$ & [131] \\
\hline Catechin & Pine tree & $\mathrm{SFE} / \mathrm{CO}_{2} ; \mathrm{SFE} / \mathrm{CO}_{2}+$ ethanol & {$[132,133]$} \\
\hline Catechin & Berries & $\mathrm{SFE} / \mathrm{CO}_{2}$ & [109] \\
\hline Catechin & Marigold (Calendula officinalis) & $\mathrm{SFE} / \mathrm{CO}_{2}+$ ethanol & [101] \\
\hline
\end{tabular}




\begin{tabular}{|c|c|c|c|}
\hline Catechin & $\begin{array}{l}\text { Hawthorn (Crataegus } \\
\text { apathulata) }\end{array}$ & $\mathrm{SFE} / \mathrm{CO}_{2}+$ ethanol & [101] \\
\hline Catechin & $\begin{array}{l}\text { Scented mayweed (Matricaria } \\
\text { recutita) }\end{array}$ & $\mathrm{SFE} / \mathrm{CO}_{2}+$ ethanol & [101] \\
\hline Catechin & Strobilanthes crispus & $\mathrm{SFE} / \mathrm{CO}_{2}+$ ethanol & [113] \\
\hline Cinnamic acid & $\begin{array}{l}\text { Olive oil residues (Olea } \\
\text { europaea) }\end{array}$ & $\mathrm{SFE} ; \mathrm{SE} / \mathrm{CO}_{2}$; ethanol & [131] \\
\hline Cinnamic acid & $\begin{array}{l}\text { Macela (Achyrocline } \\
\text { satureioides) }\end{array}$ & $\mathrm{SFE} / \mathrm{CO}_{2}, \mathrm{CO}_{2}+$ ethanol & {$[125]$} \\
\hline Cinnamic acid & Cherry (Prunus avium) & $\mathrm{SFE} / \mathrm{CO}_{2}, \mathrm{CO}_{2}+$ ethanol & [134] \\
\hline Cirsimaritin & $\begin{array}{l}\text { Rosemary (Rosmarinus } \\
\text { officinalis) }\end{array}$ & $\mathrm{SFE} / \mathrm{CO}_{2}+$ ethanol & [135] \\
\hline Corilagin & Barbados nut (Jatropha curcas) & $\mathrm{SFE} / \mathrm{CO}_{2}+$ methanol & [136] \\
\hline Coumaric acid & Brazilian propolis & $\mathrm{SFE} / \mathrm{CO}_{2}$ & {$[120]$} \\
\hline Coumaric acid & $\begin{array}{l}\text { Olive oil residues (Olea } \\
\text { europaea) }\end{array}$ & $\mathrm{SFE} ; \mathrm{SE} / \mathrm{CO}_{2}$; ethanol & [131] \\
\hline Coumarins & Rice (Oryza sativa) & $\mathrm{SFE} / \mathrm{CO}_{2}+$ ethanol & [137] \\
\hline Coumarins & Dodder (Cuscuta reflexa) & $\mathrm{SFE} / \mathrm{CO}_{2}+$ methanol & [138] \\
\hline Coumarins & $\begin{array}{l}\text { Wormwood (Artemisia } \\
\text { capillaris) }\end{array}$ & $\mathrm{SFE} / \mathrm{CO}_{2}$ & [139] \\
\hline Coumarins & Emburana (Torresea cearensis) & $\mathrm{SFE} / \mathrm{CO}_{2}$ & {$[140]$} \\
\hline Coumarins & $\begin{array}{l}\text { Sweet grass (Hierochloe } \\
\text { odorata) }\end{array}$ & $\begin{array}{l}\text { SE; MAE; SFE/organic solvents; } \\
\mathrm{CO}_{2}\end{array}$ & [141] \\
\hline Coumarins & Dodder (Cuscuta reflexa) & $\mathrm{SFE} / \mathrm{CO}_{2}+$ methanol & [138] \\
\hline Coumarins & Bai Zhi (Angelica dahurica) & $\mathrm{SFE} / \mathrm{CO}_{2}+$ ethanol & {$[142,143]$} \\
\hline Daidzein & Soybean (Glycine max) & $\begin{array}{l}\mathrm{SFE} / \mathrm{CO}_{2}+\text { methanol/water, }+ \\
\text { ethanol }\end{array}$ & {$[96,105,144]$} \\
\hline Fucocoumarin & Bishop's flower (Ammi majus) & $\mathrm{SFE} / \mathrm{CO}_{2}+$ ethanol & [145] \\
\hline Gallic acid & Rice (Oryza sativa Linn.) & $\mathrm{SFE} / \mathrm{CO}_{2}+$ ethanol & {$[137]$} \\
\hline Gallic acid & Barbados nut (Jatropha curcas) & $\mathrm{SFE} / \mathrm{CO}_{2}+$ methanol & [136] \\
\hline Galic acid & $\begin{array}{l}\text { Macela (Achyrocline } \\
\text { satureioides) }\end{array}$ & $\mathrm{SFE} / \mathrm{CO}_{2}+$ ethanol & [146] \\
\hline Ellagic acid & Barbados nut (Jatropha curcas) & $\mathrm{SFE} / \mathrm{CO}_{2}+$ methanol & [136] \\
\hline Genistein & Soybean (Glycine max) & $\begin{array}{l}\mathrm{SFE} / \mathrm{CO}_{2}+\text { methanol; water }+ \\
\text { ethanol }\end{array}$ & {$[96,105,144]$} \\
\hline Genistein & Wild Cherry (Prunus avium) & $\mathrm{SFE} / \mathrm{CO}_{2}$ & [134] \\
\hline Genistein & Grape & $\begin{array}{l}\text { ethanol/water; } \mathrm{SFE} / \mathrm{CO}_{2}+\text { ethanol, } \\
+ \text { methanol }\end{array}$ & {$[86,147,148]$} \\
\hline Genistein & Grape & $\mathrm{SFE} / \mathrm{CO}_{2} ; \mathrm{SFE} / \mathrm{CO}_{2}+$ ethanol & {$[85,87,89,128$} \\
\hline Genistein & Black currant (Ribes nigrum) & $\begin{array}{l}\mathrm{SE} ; \mathrm{SFE} / \text { methanol; } \mathrm{CO}_{2}+ \\
\text { methanol }\end{array}$ & [149] \\
\hline Genistein & Black currant (Ribes nigrum) & $\mathrm{SE} ; \mathrm{SFE} /$ ethanol; $\mathrm{CO}_{2}$ & {$[150]$} \\
\hline Genistein & Elderberry (Sambucus) & $\mathrm{SFE} / \mathrm{CO}_{2}$ & [91] \\
\hline Genistein & Sour cherry (Prunus cerasus) & $\mathrm{SFE} / \mathrm{CO}_{2}$ & [151] \\
\hline Glabridin & Licorice (Glycyrrhiza glabra) & $\mathrm{SFE} / \mathrm{CO}_{2}$ & {$[152]$} \\
\hline
\end{tabular}




\begin{tabular}{|c|c|c|c|}
\hline Isocoumarin & $\begin{array}{l}\text { Coriander (Coriandrum } \\
\text { sativum) }\end{array}$ & $\mathrm{SFE} / \mathrm{CO}_{2}$ & [153] \\
\hline Kaempferol & Strobilanthes crispus & $\mathrm{SFE} / \mathrm{CO}_{2}+$ ethanol & [113] \\
\hline Kaempferol & Tea (Camellia sinensis L.) & $\mathrm{SFE} / \mathrm{CO}_{2}$ & {$[126]$} \\
\hline Kaempferol & Rooibos (Aspalathus linearis) & $\mathrm{SFE} / \mathrm{CO}_{2}$ & {$[126]$} \\
\hline Kaempferol & $\begin{array}{l}\text { Indian gooseberry (Phyllanthus } \\
\text { emblica L.) }\end{array}$ & $\mathrm{SFE} / \mathrm{CO}_{2}+$ methanol; methanol & [149] \\
\hline Kaempferol & Black currant (Ribes nigrum) & $\begin{array}{l}\mathrm{SE} ; \mathrm{SFE} / \text { methanol; } \mathrm{CO}_{2}+ \\
\text { methanol }\end{array}$ & [149] \\
\hline Kaempferol & Black currant (Ribes nigrum) & $\mathrm{SE} ; \mathrm{SFE} /$ ethanol; $\mathrm{CO}_{2}$ & {$[150]$} \\
\hline Kampheride & Brazilian propolis & $\mathrm{SFE} / \mathrm{CO}_{2}$ & {$[120]$} \\
\hline Lignan & $\begin{array}{l}\text { Five-flavor berry (Schisandra } \\
\text { chinensis) }\end{array}$ & $\mathrm{SFE} / \mathrm{CO}_{2}, \mathrm{SC}-\mathrm{CO}_{2}+$ ethanol & [154] \\
\hline Lignan & $\begin{array}{l}\text { Five-flavor berry (Schisandra } \\
\text { chinensis) }\end{array}$ & USE; $\mathrm{SFE} / \mathrm{CO}_{2}, \mathrm{CO}_{2}+$ ethanol & {$[155]$} \\
\hline Lignan & Forsythia species & $\begin{array}{l}\mathrm{SE} ; \mathrm{SFE} / \text { ethanol, methanol; } \mathrm{CO}_{2} \text {, } \\
+ \text { methanol, + ethanol }\end{array}$ & [156] \\
\hline Lignan & Flax (Linum usitatissimum) & $\mathrm{SFE} / \mathrm{CO}_{2}+$ ethanol & {$[157]$} \\
\hline L-DOPA & Mucuna & $\mathrm{SFE} / \mathrm{CO}_{2}$ & {$[158]$} \\
\hline Myricetin & Crowberry (Empetrum nigrum) & $\mathrm{SE}, \mathrm{SFE} /$ ethanol, $\mathrm{CO}_{3}$ & [159] \\
\hline Myricetin & Tea (Camellia sinensis L.) & $\mathrm{SFE} / \mathrm{CO}_{2}$ & {$[126]$} \\
\hline Myricetin & Rooibos (Aspalathus linearis) & $\mathrm{SFE} / \mathrm{CO}_{2}$ & {$[126]$} \\
\hline Myricetin & Spearmint (Mentha spicata L.) & $\mathrm{SFE} / \mathrm{CO}_{2}$ & {$[118,119]$} \\
\hline Naringenin & Aspen (Populus tremula) & PHWE & [160] \\
\hline Naringenin & Spearmint (Mentha spicata L.) & $\mathrm{SFE} / \mathrm{CO}_{2}$ & {$[118,119]$} \\
\hline Naringin & Grapefruit (Citrus paradisi) & $\mathrm{SFE} / \mathrm{CO}_{2}+$ ethanol & [161] \\
\hline Orientin & Maypop (Passiflora incarnata) & $\begin{array}{l}\mathrm{SFE} / \mathrm{CO}_{2}+\text { ethanol, + methanol, } \\
+\mathrm{CHCl}^{3}\end{array}$ & [82] \\
\hline Orientin & Pigeon pea (Cajanus cajan L.) & $\mathrm{SFE} / \mathrm{CO}_{2}$ & [162] \\
\hline Orotinin & Patrinia villosa Juss. & $\mathrm{SFE} / \mathrm{CO}_{2}+$ ethanol & [163] \\
\hline Osthole & Cnidium monnieri & $\mathrm{SFE} / \mathrm{CO} 2$ & [164] \\
\hline Pinostrobin & Pigeon pea (Cajanus cajan L.) & $\mathrm{SFE} / \mathrm{CO}_{2}$ & [162] \\
\hline Polyphenols & Cocoa & $\mathrm{SFE} / \mathrm{CO}_{2}+$ ethanol & {$[165]$} \\
\hline Quercetin & Crowberry (Empetrum nigrum) & $\mathrm{SE}, \mathrm{SFE} /$ ethanol, $\mathrm{CO}_{2}$ & [159] \\
\hline Quercetin & Tea (Camellia sinensis L.) & $\mathrm{SFE} / \mathrm{CO}_{2}$ & {$[126]$} \\
\hline Quercetin & Rooibos (Aspalathus linearis) & $\mathrm{SFE} / \mathrm{CO}_{2}$ & {$[126]$} \\
\hline Quercetin & Onion (Allium cepa) & $\begin{array}{l}\mathrm{SFE} ; \mathrm{SWE} / \mathrm{CO}_{2}+\text { ethanol; SFE/ } \\
\mathrm{CO}_{2}\end{array}$ & {$[166,167]$} \\
\hline Quercetin & Hypericum & $\mathrm{SFE} / \mathrm{CO}_{2}+$ methanol & {$[100]$} \\
\hline Quercetin & Grape & $\mathrm{SFE} / \mathrm{CO}_{2}+$ ethanol & [84] \\
\hline Quercetin & $\begin{array}{l}\text { Macela (Achyrocline } \\
\text { satureioides) }\end{array}$ & $\mathrm{SFE} / \mathrm{CO}_{2}+$ ethanol & [146] \\
\hline Resveratol & Grape & $\mathrm{SFE} / \mathrm{CO}_{2}+$ ethanol & [84] \\
\hline Resveratol & Polygonum cuspidatum & $\mathrm{SFE} / \mathrm{CO}_{2}+$ ethanol & [168] \\
\hline Resveratol & Hop (Humulus lupulus) & $\mathrm{SFE} / \mathrm{CO}_{2}$ & [169] \\
\hline
\end{tabular}




\begin{tabular}{|c|c|c|c|}
\hline Rhamnetin & Marigold (Calendula officinalis) & $\mathrm{SFE} / \mathrm{CO}_{2}+$ ethanol & {$[101]$} \\
\hline Rhamnetin & $\begin{array}{l}\text { Hawthorn (Crataegus } \\
\text { spathulata) }\end{array}$ & $\mathrm{SFE} / \mathrm{CO}_{2}+$ ethanol & {$[101]$} \\
\hline Rhamnetin & $\begin{array}{l}\text { Scented mayweed (Matricaria } \\
\text { recutita) }\end{array}$ & $\mathrm{SFE} / \mathrm{CO}_{2}+$ ethanol & {$[101]$} \\
\hline Rutin & Tea (Camellia sinensis L.) & $\mathrm{SFE} / \mathrm{CO}_{2}$ & {$[126]$} \\
\hline Rutin & Rooibos (Aspalathus linearis) & $\mathrm{SFE} / \mathrm{CO}_{2}$ & {$[126]$} \\
\hline Rutin & Elderberry (Sambucus nigra) & ASE & {$[170]$} \\
\hline Rutin & Olive (Olea europaea) & $\mathrm{SFE} / \mathrm{CO}_{2}$ & {$[112]$} \\
\hline Rutin & Spearmint (Mentha spicata L.) & $\mathrm{SFE} / \mathrm{CO}_{2}$ & {$[118,119]$} \\
\hline Rutin & Strobilanthes crispus & $\mathrm{SFE} / \mathrm{CO}_{2}+$ ethanol & {$[113]$} \\
\hline Rutin & $\begin{array}{l}\text { Passionflower (Passiflora } \\
\text { incarnata) }\end{array}$ & $\begin{array}{l}\mathrm{SFE} / \mathrm{CO}_{2}+\text { ethanol, + methanol, } \\
+\mathrm{CHCl}_{3}\end{array}$ & {$[82]$} \\
\hline Scutellarein & $\begin{array}{l}\text { Rosemary } \\
\text { (Rosmarinus officinalis) }\end{array}$ & $\mathrm{SFE} / \mathrm{CO}_{2}+$ ethanol & {$[135]$} \\
\hline Stilbenes & Pigeon pea (Cajanus cajan L.) & $\mathrm{SFE} / \mathrm{CO}_{2}$ & {$[162]$} \\
\hline Tannin & Canola (Brassica napus L.) & $\begin{array}{l}\mathrm{SE} ; \mathrm{SFE} / \text { hexane; } \mathrm{CO}_{2}, \mathrm{SC}-\mathrm{CO}_{2}+ \\
\text { ethanol }\end{array}$ & {$[171,172]$} \\
\hline Tannin & $\begin{array}{l}\text { Stonebreaker (Phyllanthus } \\
\text { niruri) }\end{array}$ & $\begin{array}{l}\text { SE; SFE; PWHE/organic solvents; } \\
\mathrm{CO} 2 ; \mathrm{H} 2 \mathrm{O}\end{array}$ & {$[173,174]$} \\
\hline Thymoquinone & Black cumin (Nigella sativa) & $\mathrm{SFE} / \mathrm{CO}_{2}+$ ethanol & {$[175]$} \\
\hline Vitexin & Maypop (Passiflora incarnata) & $\begin{array}{l}\mathrm{SFE} / \mathrm{CO}_{2}+\text { ethanol, + methanol, } \\
+\mathrm{CHCl} 3\end{array}$ & {$[82]$} \\
\hline Vitexin & Pigeon pea (Cajanus cajan L.) & $\mathrm{SFE} / \mathrm{CO}_{2}$ & {$[162]$} \\
\hline Wogonin & $\begin{array}{l}\text { Blue skullcap (Scutellaria } \\
\text { lateriflora) }\end{array}$ & $\begin{array}{l}\mathrm{SFE} ; \mathrm{SubWE} / \mathrm{CO}_{2}+\text { ethanol; } \\
\text { water }\end{array}$ & {$[97]$} \\
\hline Wogonin & Skullcap (Scutellariae radix) & $\mathrm{SE} ; \mathrm{SFE} / \mathrm{CO}_{2}+$ methanol & {$[124]$} \\
\hline Xanthone & $\begin{array}{l}\text { Mangosteen (Garcinia } \\
\text { mangostana) }\end{array}$ & $\mathrm{SFE} / \mathrm{CO}_{2}+$ ethanol & {$[176-178]$} \\
\hline$\gamma$-oryzanol & Rice (Oryza sativa) & $\mathrm{SFE} / \mathrm{CO}_{2}$ & {$[179-181]$} \\
\hline N.D. & $\begin{array}{l}\text { Bitter melon (Momordica } \\
\text { charantia) }\end{array}$ & $\mathrm{SFE} / \mathrm{CO}_{2}+$ ethanol & {$[182]$} \\
\hline N.D. & Pomelo (Citrus grandis) & $\mathrm{SFE} / \mathrm{CO}_{2}+$ ethanol & {$[183]$} \\
\hline
\end{tabular}

Flavonoids are phenolic compounds that serve as antioxidants and are found in plants as pigments [80]. There are many flavonoid classes; the most common are flavanes, flavones, flavanones, flavonols, isoflavonoids, and anthocyanins. Their structure is usually characterized by a C6-C3-C6 carbon skeleton with double bonds and attached oxygen functional groups [81]. One of the earliest studies in this field has been done by Moraes et al. [82], where the isolation of glycosylated flavonoids from Passiflora edulis has been carried out. One of potential sources of flavonoids is grape and its by-pruducts. Several authors researched the extraction of flavonoids from grape and grape by-products [83-93]. Results obtained from these researches can majorly contribute to grape-processing industries and wineries in terms of giving information on how to achieve a higher recovery of some flavonids extracted with SFE, besides those obtained in grape processing. Flavonoids are polar compounds, and because of this, there are only few studies on their separation 
with pure $\mathrm{CO}_{2}$. Such example is the isolation of the moderately polar apigenin from chamomile, where the investigations showed that extraction with $\mathrm{SC}-\mathrm{CO}_{2}$ is more rapid than other conventional methods [94].

Because of the polarity of flavonoids, polar modifiers have to be added to $\mathrm{SC}-\mathrm{CO}_{2}$ to increase solubility. Numerous researches were performed, where the influence of modifiers was investigated [84, 90, 94-107]. These studied showed that the addition of a modifier generally increases the efficiency of extraction by increasing yields. However, too much co-solvent used is not economical, due to the higher amount of energy required for its removal.

As mentioned, mainly $\mathrm{CO}_{2}$ is used for SFE, but other fluids could be used as well. Chiu et al. [99] investigated the extraction of flavonoids from ginkgo using $\mathrm{CO}_{2}, \mathrm{~N}_{2} \mathrm{O}$, and Freon 1,1,1,2-tetrafluoroethane (R134a). Each supercritical medium itself could not extract flavonoids from dried ginkgo leaves in this case. Successful extraction was achieved with the addition of ethanol as modifier [99].

Before undertaking an individual SFE process, often a sample preparation is required. Antolovich et al. [95] reviewed techniques in the preparation of samples for the extraction of phenolic compounds from fruits, then described the analytical methods for the determination of phenolic compounds present in the processed material. The particle size of the material played an important role. Liu et al. [108] investigated the influence of the particle size of $M$. stigma on extraction yields of flavonoids. When particle size is reduced, contact area is increased; hence yields got higher. Oppositely, when the particles are too small, this lowers the extraction yield, due to agglomeration [108].

Not only SFE but also pressurized hotwater extraction (PHWE) [184, 185] and accelerated solvent extraction (ASE) [170] could be applied in order to ensure sustainability. Ollanketo et al. [185] investigated PHWE of antioxidant compounds (among them were also flavonoids) from sage (Salvia officinalis). This method is promising and presents an alternative to conventional processes; however, the disadvantage is thermal degradation of thermolabile compounds.

Senorans et al. [186] and Simo et al. [187] carried out countercurrent supercritical fluid extraction (CC-SFE) of flavonoids from orange juice. After extraction, further separation of flavonoids with reversed-phase liquid chromatography (RP-LC) and/or micellar electrokinetic chromatography (MEKC) was performed in order to fractionate compounds. CC-SFE was performed in extraction column filled with spherical steel packing. This packing material provides a larger contact area, which allows more efficient separations [186]. This technique is promising for the separation of other nutraceuticals from liquid samples (e.g. extracts).

SFE was applied to sample preparation of Rhodiola rosea, and further analysis of flavonoids was performed by liquid chromatography in tandem with mass spectroscopy [188]. Flavonoids are the most often determined and identified by chromatographic methods such as thin layer chromatography (TLC) [189], RP-LC with C8- or C18-bonded silica column packing. Liquid chromatography could be coupled with mass spectroscopy (LC-MS). Those methods are usually applied with a diode array detector (DAD). High-performance liquid chromatography (HPLC) could also be coupled with electrospray ionization detection (ESI), or light-scattering detection (LSD). Another way of identifying compounds is gas chromatography (GC) coupled with mass spectroscopy (GC-MS). Detailed identification and determination methods of various phenolic compounds are described in the literature [135, 141, 190-192]. Klejdus et al. [193] investigated a new analytic approach by using supercritical fluids, i.e. solid-phase/ supercritical fluid extraction (SPE/SFE), which provides a more efficient isolation and identification of simple phenols and phenolic acids as only by using $\mathrm{CO}_{2}$. Crego et al. [194] proposed subcritical water extraction (SubWE) of phe- 
nolic compounds from rosemary in combination with capillary electrophoresis (CE). The water used in those processes contributed to the sustainability of said processes. The application of SubWE is useful for the isolation of more polar compounds, and extraction could be achieved faster in a less viscous extraction medium.

The utilization of renewable resources, e.g. waste from the forestry industry, is becoming more attractive. Therefore, Yesil-Celiktas et al. [133] proposed the recovery of some flavonoids from pine barks using SFE with $\mathrm{CO}_{2}$.

Flavonoids, as known, help to prevent diseases with their antioxidant, antiviral, antimicrobial, and cancer prevention properties; therefore they could be used as medicines. Miao et al. [195] studied SFE of flavonoids from ginkgo biloba leaves with $\mathrm{CO}_{2}$ and ethanol as co-solvent, coupled with micronization, in order to obtain smaller particles for better drug release in the body.

The future trend of SFE investigations is scaled up from an analytical scale to preparative or even an industrial scale. Such processes have to be optimized in order to achieve costeffective operations. Therefore solvents shall be recycled. Often organic solvents are used as modifiers of $\mathrm{SC}-\mathrm{CO}_{2}$. In order to achieve successful recycling, evaporation of the modifier is required. The evaporation step consumes a great amount of energy. If an extract is gathered in a collective trap, filled with a co-solvent, the evaporative step could be avoided, which presents a more economical solution [88].

Although SFE serves mainly for the isolation of certain compounds from plant matrix, it could be used for the pretreatment of sample preparations for further processing. Xu et al. [196] observed that SFE could break cell walls in order to extract lysed bee pollen oils from rape bee pollen more efficiently. Compounds in the extract could be separated afterward with chromatographic methods. Supercritical fluid chromatography (SFC) is one of many chromatographic methods that were rarely used in the past, but lately it has become more attractive. Ramirez et al. [197],
[198] studied rosemary extraction followed by SFC. The extracted compounds could be analysed withouth further sample preparation (online analysis) if analytical SFC is applied, or even fractionationated in the case of preparative SFC (prep SFC) [197].

Generally, the solubility of a compound in a solvent has to be known in order to optimize the extraction and fractionation of the solutes. The importance of solubility data could be observed in the work of Nunes et al. [199], where a study of the extraction of phenolic compounds (catechin, qurcetin-3-glycoside, coumaric acid, resveratol) from water-alcohol mixtures (such as wine) was conducted. At elevated pressures, water is poorly miscible with $\mathrm{SC}-\mathrm{CO}_{2}$; on the other hand, ethanol is completely miscible above supercritical conditions. Therefore two phases are formed when pressurized $\mathrm{CO}_{2}$ is introduced into a water-ethanol mixture. Observations of Nunes et al. [199] confirmed that the ethanolrich phase contains more phenolic compounds. This advantage could be used for the separation of flavonoids from alcoholic beverages, such as wine.

SCF can be applied as an alternative solution for the extraction of oils from biomass, rich with several phenolics, esters, and furan derivatives. Such extraction was investigated by Chumpoo and Prasassarakich [200], where supercritical ethanol in inert $\mathrm{H}_{2}$ atmosphere was used for the extraction of oils from biomass. This study provides knowledge about supercritical ethanol extraction, where it's mandatory to use inert atmosphere to avoid combustion. Supercritical ethanol extraction is like SubWE, an appropriate alternative to SFE, where mainly modified $\mathrm{CO}_{2}$ is used, because it can separate polar compounds more effectively. On the other hand, this method is not as economical as SFE with $\mathrm{CO}_{2}$ because of the higher price of ethanol.

Flavonoids cover a wide range of phenolic compounds, but there are other phenolic compounds in the natural matrices that affect human health. They are grouped into the following categories (besides flavonoids): simple phenols, benzoic acid derivates, stilbenes, tan- 
nins, lignans, and lignins [201]. Some of those compounds were extracted by SFE-e.g. phenylacetic acids [137]; cinnamic acids, coumarins, isocoumarins $[138-143,145,153,164,174$, 202], and chromones; lignans [155-157]; tannins [171, 172]; xanthones [178] and stilbenes [64, 84, 106, 168, 169, 189]; and betacyanins [203]. In the work of Wang et al. [204], SFE combined with liquid-liquid extraction (LLE) for the separation of coumarins from Angelica dahurica is presented. First, SFE of material was performed, and the extract obtained was afterwards extracted by LLE. SFE extract was diluted in petroleum ether. The prepared sample was extracted first with water, then with a $60 \%$ methanol/water solution with LLE. Selectivity and separation efficiency were better than with SFE alone [204]. Phenyllpropanoides, benzoic acid derivates, coumarins, and isocoumarins are very important from a pharmacological point of view, because they prevent diseases and have antioxidant, antimicrobial, anti-inflammatory, and cancer-preventive properties. Coumarins could also help in treating AIDS, among other properties.

\subsection{Essential oils}

Investigations of SFE of essential oils from natural matter are listed in Table 4.

Essential oils are volatile and liquid aroma components present in plant materials. Often they are poorly soluble in water and have characteristic odors. Therefore, they are used in food flavoring and perfumery.

Essential oils are mostly separated with hydrodistillation (HD), including steam distillation (SD) [205]. Many investigations and comparisons between conventional and newer separation processes (as SFE, PHWE, microwave-assisted hydrodistillation [MAHD], and solid-phase micro extraction [SPME]) were done [206-215]. Results obtained showed that conventional processes in general give higher global yields. On the other hand, newer processes, such as SFE, provide faster separation. The properties of a solvent in SFE could be manipulated by just adjusting operation parameters, and a wide range of applications is possible. However, SFE is not always the optimal solution, because the solubility of a desired component in supercritical media is not satisfactory in that case. Even more, the depressurization step of $\mathrm{CO}_{2}$ and the renewed repressurization of SFE is a big disadvantage, because more energy is consumed. Carlson et al. [216] proposed the use of reverse-osmosis membranes to avoid instantaneous expansion and to lower operating costs.

Undesired racemization, where risky enantiomers are included in the racemic mixture, could be avoided [217]. First, the plant material is pretreated with SFE and then is extracted and further fractionated with SFC in chiral mode. This procedure is appropriate for the isolation of bioactive compounds, which could be added in food, which requires higher purity, or could be used as medicine, on the other hand.

The main application of SFE in the field of essential oils is the isolation of terpenes (sesquiterpenes) or terpenoids from plant material. When the presence of terpenes and terpenoids is not desired in the final products, the process is called deterpenation [218-220]. Mostly, $\mathrm{CO}_{2}$ is applied for SFE of essential oils, but ethane could be a great alternative, because its solvent power for the extraction of essential oils is higher, which is desired in the deterpenation process [221]. 
Table 4

Application of SFE for the separation of phenolic compounds from plants

\begin{tabular}{|c|c|c|c|}
\hline Compounds & Plant material ( Biological name) & Method/solvent & Reference(s) \\
\hline 1,8-Cineole & Swamp mallet (Eucalyptus spathulata) & $\mathrm{HD} ; \mathrm{SFE} / \mathrm{CO}_{2}$ & {$[222]$} \\
\hline 1,8-Cineole & Coolabah (Eucalyptus microtheca) & $\mathrm{HD} ; \mathrm{SFE} / \mathrm{CO}_{2}$ & {$[222]$} \\
\hline 1,8-Cineole & $\begin{array}{l}\text { French lavender } \\
\text { (Lavandula x intermedia Grosso) }\end{array}$ & $\mathrm{SFE} / \mathrm{CO}_{2}$ & [217] \\
\hline 1,8-Cineole & $\begin{array}{l}\text { Lavandin abrial } \\
\text { (Lavandula } x \text { intermedia Abrial) }\end{array}$ & $\mathrm{SFE} / \mathrm{CO}_{2}$ & [217] \\
\hline 1,8-Cineole & $\begin{array}{l}\text { Lavandin Puper } \\
\text { (Lavandula } x \text { intermedia Super) }\end{array}$ & $\mathrm{SFE} / \mathrm{CO}_{2}$ & [217] \\
\hline 1,8-Cineole & Sage (Salvia officinalis L.) & $\mathrm{HD} ; \mathrm{SFE} / \mathrm{CO}_{2}$ & {$[223-226]$} \\
\hline 1,8-Cineole & French lavender (Lavandula stoechas) & HD; SubWE/H2O & [209] \\
\hline 1,8-Cineole & Khoa (Satureja boliviana) & $\mathrm{SFE} / \mathrm{CO}_{2}+$ ethanol & {$[146]$} \\
\hline 1,8-Cineole & $\begin{array}{l}\text { Cotton lavender } \\
\text { (Santolina chamaecyparissus) }\end{array}$ & $\mathrm{HD} ; \mathrm{SFE} / \mathrm{CO}_{2}$ & [227] \\
\hline 1,8-Cineole & Sweet bay (Laurus nobilis L.) & $\mathrm{SFE} / \mathrm{CO}_{2}$ & {$[228,229]$} \\
\hline 1,8-Cineole & Saigon cinnamon (Cinnamomum loureiroi) & $\mathrm{SFE} / \mathrm{CO}_{2}$ & {$[230]$} \\
\hline 1,8-Cineole & Clove basil (Ocimum gratissimum) & $\mathrm{SFE} / \mathrm{CO}_{2}$ & [231] \\
\hline 1,8-Cineole & Argyle apple (Eucalyptus cinerea) & $\mathrm{HD} ; \mathrm{SFE} / \mathrm{CO}_{2}$ & [232] \\
\hline 1,8-Cineole & Sweet gale (Myrica gale L.) & $\mathrm{SFE} / \mathrm{CO}_{2}$ & [233] \\
\hline 1,8-Cineole & French lavender (Lavandula stoechas) & $\mathrm{SFE} / \mathrm{CO}_{2}$ & {$[220]$} \\
\hline 1,8-Cineole & Peppermint (Mentha piperita) & $\mathrm{SFE} / \mathrm{CO}_{2}$ & [234] \\
\hline 4-Nonanone & (Capillipedium parviflorum) & $\mathrm{HD} ; \mathrm{SFE} / \mathrm{CO}_{2}$ & [235] \\
\hline 4-Nonanol & (Capillipedium parviflorum) & $\mathrm{HD} ; \mathrm{SFE} / \mathrm{CO}_{2}$ & {$[235]$} \\
\hline 4-Terpineol & Black cumin (Nigella sativa L.) & $\mathrm{MAE} ; \mathrm{SFE} / \mathrm{CO}_{2}$ & {$[202]$} \\
\hline 4-Undecanone & (Capillipedium parviflorum) & $\mathrm{HD} ; \mathrm{SFE} / \mathrm{CO}_{2}$ & [235] \\
\hline 4-Undecanol & (Capillipedium parviflorum) & $\mathrm{HD} ; \mathrm{SFE} / \mathrm{CO}_{2}$ & {$[235]$} \\
\hline Artemisinin & Sweet wormwood (Artemisia annua) & $\mathrm{SFE} / \mathrm{CO}_{2}+$ ethanol & [236] \\
\hline Bicyclogermacrene & Peruvian pepper (Schinus molle) & $\mathrm{SFE} / \mathrm{CO}_{2}$ & [237] \\
\hline Boldine & Boldo (Peumus boldus) & PHWE; SFE/CO & [208] \\
\hline Cadinol & Marigold (Calendula officinalis) & $\mathrm{SFE} / \mathrm{CO}_{2}$ & {$[238]$} \\
\hline Camphor & $\begin{array}{l}\text { Lavandin abrial } \\
\text { (Lavandula } x \text { intermedia Abrial) }\end{array}$ & $\mathrm{SFE} / \mathrm{CO}_{2}$ & [217] \\
\hline Camphor & Sage (Salvia officinalis) & $\mathrm{SFE} / \mathrm{CO}_{2}$ & [223] \\
\hline Camphor & Sage (Salvia officinalis L.) & $\mathrm{HD} ; \mathrm{SFE} / \mathrm{CO}_{2}$ & {$[223-226]$} \\
\hline Camphor & French lavender (Lavandula stoechas) & $\mathrm{HD} ; \mathrm{SubWE} / \mathrm{H}_{2} \mathrm{O}$ & [209] \\
\hline Camphor & $\begin{array}{l}\text { Cotton lavender } \\
\text { (Santolina chamaecyparissus) }\end{array}$ & $\mathrm{HD} ; \mathrm{SFE} / \mathrm{CO}_{2}$ & [227] \\
\hline Camphor & (Thymus lotocephalus G. Lopez \& R. Morales) & $\mathrm{SFE} / \mathrm{CO}_{2}$ & [239] \\
\hline Camphor & Borage (Borago officinalis) & $\begin{array}{l}\mathrm{HD} ; \mathrm{SFE} / \mathrm{CO}_{2}+ \\
\text { methanol }\end{array}$ & {$[240]$} \\
\hline Camphor & French lavender (Lavandula stoechas) & $\mathrm{SFE} / \mathrm{CO}_{2}$ & [220] \\
\hline
\end{tabular}




\begin{tabular}{|c|c|c|c|}
\hline Camphor & Lavender (Lavandula angustifolia) & $\mathrm{SFE} / \mathrm{CO}_{2}$ & {$[241,242]$} \\
\hline Camphor & Lavender (Lavandula viridis) & $\mathrm{HD} ; \mathrm{SFE} / \mathrm{CO}_{2}$ & {$[243]$} \\
\hline Carnosic acid & Rosemary (Rosmarinus officinalis L.) & $\mathrm{SFE} / \mathrm{CO}_{2}+$ ethanol & $\begin{array}{l}{[197,212,225,} \\
244-250]\end{array}$ \\
\hline Carnosic acid & Rosemary (Rosmarinus officinalis L.) & SubWE & {$[184,194]$} \\
\hline Carnosic acid & Rosemary (Rosmarinus officinalis L.) & $\mathrm{SFE} / \mathrm{CO}_{2}$ & {$[251-253]$} \\
\hline Carnosic acid & Rosemary (Rosmarinus officinalis L.) & $\begin{array}{l}\text { SFE; } \mathrm{PLE} / \mathrm{CO}_{2} ; \mathrm{H}_{2} \mathrm{O} \text {, } \\
\text { ethanol }\end{array}$ & {$[254]$} \\
\hline Carnosol & Rosemary (Rosmarinus officinalis L.) & $\mathrm{SFE} / \mathrm{CO}_{2}+$ ethanol & $\begin{array}{l}{[197,212,225,} \\
244-250]\end{array}$ \\
\hline Carnosol & Rosemary (Rosmarinus officinalis L.) & SubWE & {$[184,194]$} \\
\hline Carnosol & Rosemary (Rosmarinus officinalis L.) & $\mathrm{SFE} / \mathrm{CO}_{2}$ & {$[251-253]$} \\
\hline Carnosol & Rosemary (Rosmarinus officinalis L.) & $\begin{array}{l}\text { SFE; PLE/CO } ; \text {; water, } \\
\text { ethanol }\end{array}$ & {$[254]$} \\
\hline Carvacrol & Oregano (Origanum vulgare) & $\mathrm{SFE} / \mathrm{CO}_{2}$ & {$[218,255,256]$} \\
\hline Carvacrol & Summer savory (Satureja hortensis) & $\begin{array}{l}\mathrm{HD}, \mathrm{SFE} / \mathrm{CO}_{2}+ \\
\text { methanol }\end{array}$ & {$[257]$} \\
\hline Carvacrol & Winter savory (Satureja montana) & $\mathrm{HD} ; \mathrm{SFE} / \mathrm{CO}_{2}$ & {$[258]$} \\
\hline Carvacrol & Lippia origanoides & $\mathrm{SFE} / \mathrm{CO}_{2}$ & {$[214]$} \\
\hline Carvacrol & Black cumin (Nigella sativa L.) & $\mathrm{MAE} ; \mathrm{SFE} / \mathrm{CO}_{2}$ & {$[202]$} \\
\hline Carvone & Indian dill (Anethum sowa) & $\mathrm{HD} ; \mathrm{SFE} / \mathrm{CO}_{2}$ & {$[259]$} \\
\hline Carvone & Spearmint (Mentha spicata) & $\begin{array}{l}\mathrm{SE} ; \mathrm{SFE} / \mathrm{hexane}, \mathrm{DCM} \text {, } \\
\text { ethanol; } \mathrm{CO}_{2}\end{array}$ & {$[260]$} \\
\hline Caryophyllene & Curry plant (Helichrysum italicum) & $\mathrm{HD} ; \mathrm{SFE} / \mathrm{CO}_{2}$ & {$[261]$} \\
\hline Caryophyllene & Khoa (Satureja boliviana) & $\mathrm{SFE} / \mathrm{CO}_{2}+$ ethanol & {$[262]$} \\
\hline Caryophyllene & Oregano (Origanum vulgare) & $\mathrm{SFE} / \mathrm{CO}_{2}$ & {$[218,255,256]$} \\
\hline Caryophyllene & Oregano (Origanum vulgare) & SubWE & {$[263]$} \\
\hline Caryophyllene & Peruvian pepper (Schinus molle) & $\mathrm{SFE} / \mathrm{CO}_{2}$ & {$[237]$} \\
\hline Caryophyllene & Indian gooseberry (Phyllanthus emblica L.) & $\mathrm{SFE} / \mathrm{CO}_{2}$ & {$[149]$} \\
\hline Caryophyllene & Madeira mahogany (Persea indica) & $\mathrm{SFE} / \mathrm{CO}_{2}+$ ethanol & {$[264]$} \\
\hline Caryophyllene & Honeyherb (Lippia dulcis) & $\mathrm{HD} ; \mathrm{SFE} / \mathrm{CO}_{2}$ & {$[265]$} \\
\hline Caryophyllene & $\begin{array}{l}\text { Sri Lanka cinnamon } \\
\text { (Cinnamomum zeylanicum) }\end{array}$ & $\mathrm{SFE} / \mathrm{CO}_{2}$ & {$[266]$} \\
\hline Cedrene & Dragonhead (Dracocophalum tanguticum) & $\mathrm{SFE} / \mathrm{CO}_{2}$ & {$[267]$} \\
\hline $\begin{array}{l}\text { Chrysanthenol } \\
\text { acetate }\end{array}$ & Wormwood (Artemisia absinthium L.) & $\mathrm{SFE} / \mathrm{CO}_{2}+$ ethanol & {$[268]$} \\
\hline Cinnamonaldehyde & Cinnamon (Cinnamomum verum) & $\mathrm{HD} ; \mathrm{SFE} / \mathrm{CO}_{2}$ & {$[269,270]$} \\
\hline Cinnamonaldehyde & Saigon cinnamon (Cinnamomum loureiroi) & $\mathrm{SFE} / \mathrm{CO}_{2}$ & {$[230]$} \\
\hline Cinnamonaldehyde & $\begin{array}{l}\text { Sri Lanka cinnamon } \\
\text { (Cinnamomum zeylanicum) }\end{array}$ & $\mathrm{SFE} / \mathrm{CO}_{2}$ & {$[266]$} \\
\hline Citral & Lemongrass (Cymbopogon citrates) & $\mathrm{SD} ; \mathrm{SFE} / \mathrm{CO}_{2}$ & {$[271]$} \\
\hline Citronellol & Carqueja (Baccharis genistelloides) & $\mathrm{SFE} / \mathrm{CO}_{2}$ & {$[272]$} \\
\hline Copaene & Saigon Cinnamon (Cinnamomum loureiroi) & $\mathrm{SFE} / \mathrm{CO}_{2}$ & {$[230]$} \\
\hline Copaene & Honeyherb (Lippia dulcis) & $\mathrm{HD} ; \mathrm{SFE} / \mathrm{CO}_{2}$ & {$[265]$} \\
\hline Curcumene & Curry plant (Helichrysum italicum) & $\mathrm{HD} ; \mathrm{SFE} / \mathrm{CO}_{2}$ & {$[261]$} \\
\hline
\end{tabular}




\begin{tabular}{|c|c|c|c|}
\hline Cycloartenol & Carrot (Daucus carota) & $\mathrm{SFE} / \mathrm{CO}_{2}$ & [273] \\
\hline Dihydrocarvone & Indian dill (Anethum sowa) & $\mathrm{HD} ; \mathrm{SFE} / \mathrm{CO}_{2}$ & [259] \\
\hline Dillapiole & Indian dill (Anethum sowa) & $\mathrm{HD} ; \mathrm{SFE} / \mathrm{CO}_{2}$ & [259] \\
\hline Dillapiole & (Diplotaenia cachrydifolia) & $\mathrm{SFE} / \mathrm{CO}_{2}+$ ethanol & [274] \\
\hline E-ocimenone & Marigold (Tagetes minuta) & $\mathrm{SFE} / \mathrm{CO}_{2}$ & [223] \\
\hline Epoxyocimene & Wormwood (Artemisia absinthium L.) & $\mathrm{SFE} / \mathrm{CO}_{2}+$ ethanol & {$[268]$} \\
\hline Eugenol & Clove (Eugenia caryophyllata) & $\mathrm{HD} ; \mathrm{SFE} / \mathrm{CO}_{2}$ & {$[275]$} \\
\hline Eugenol & Clove (Syzygium aromaticum) & $\mathrm{SFE} / \mathrm{CO}_{2}$ & {$[255,276]$} \\
\hline Eugenol & Clove basil (Ocimum gratissimum) & $\mathrm{SFE} / \mathrm{CO}_{2}$ & [231] \\
\hline Eugenol & $\begin{array}{l}\text { Sri Lanka cinnamon } \\
\text { (Cinnamomum zeylanicum) }\end{array}$ & $\mathrm{SFE} / \mathrm{CO}_{2}$ & [266] \\
\hline Eugenol & Purple nutsedge (Cyperus rotundus) & $\mathrm{SFE} / \mathrm{CO}_{2}$ & [277] \\
\hline Eugenyl acetate & Clove (Syzygium aromaticum) & $\mathrm{SFE} / \mathrm{CO}_{2}$ & {$[255,276]$} \\
\hline $\mathrm{E}-\beta$-ocimene & Southern cone marigold (Tagetes minuta) & $\mathrm{SFE} / \mathrm{CO}_{2}$ & [223] \\
\hline Farnesene & Annual herbs (Nepeta persica) & $\begin{array}{l}\mathrm{SFE} / \mathrm{CO}_{2}+\text { methanol; } \\
\mathrm{HD}\end{array}$ & [278] \\
\hline Fenchon & French lavender (Lavandula stoechas) & $\mathrm{HD} ; \mathrm{SubWE} / \mathrm{H}_{2} \mathrm{O}$ & [209] \\
\hline Fenchon & Lavender (Lavandula angustifolia) & $\mathrm{SFE} / \mathrm{CO}_{2}$ & {$[241,242]$} \\
\hline Geranial & $\begin{array}{l}\text { Moldavian dragonhead } \\
\text { (Dracocophalum moldavica L.) }\end{array}$ & $\mathrm{SFE} / \mathrm{CO}_{2}$ & [210] \\
\hline Geraniol & $\begin{array}{l}\text { Moldavian dragonhead } \\
\text { (Dracocophalum moldavica L.) }\end{array}$ & $\mathrm{SFE} / \mathrm{CO}_{2}$ & [210] \\
\hline Geraniol & $\begin{array}{l}\text { Scented geranium } \\
\text { (Pelargonium graveolens) }\end{array}$ & $\mathrm{HD} ; \mathrm{SFE} / \mathrm{CO}_{2}$ & [279] \\
\hline Germacrone & Dragonhead (Dracocophalum tanguticum) & $\mathrm{SFE} / \mathrm{CO}_{2}$ & [267] \\
\hline Hentriacontano & Abajeru (Chrysobalanus Icaco) & $\mathrm{SE} ; \mathrm{SFE} /$ ethanol; $\mathrm{CO}_{2}$ & {$[280]$} \\
\hline Hernandulcin & Honeyherb (Lippia dulcis) & $\mathrm{HD} ; \mathrm{SFE} / \mathrm{CO}_{2}$ & [265] \\
\hline Incensole & Boswellia (Boswellia carterii) & $\mathrm{HD} ; \mathrm{SFE} / \mathrm{CO}_{2}$ & [281] \\
\hline Incensole acetate & Boswellia (Boswellia carterii) & $\mathrm{HD} ; \mathrm{SFE} / \mathrm{CO}_{2}$ & [281] \\
\hline Isomenthyl acetate & Peppermint (Metha piperita) & $\mathrm{SFE} / \mathrm{CO}_{2}$ & [223] \\
\hline Khusimol & Vetiver (Chrysopogon zizaniodes) & $\mathrm{SFE} / \mathrm{CO}_{2}+$ ethanol & [282] \\
\hline Limonene & (Diplotaenia cachrydifolia) & $\mathrm{SFE} / \mathrm{CO}_{2}+$ ethanol & {$[274,283]$} \\
\hline Limonene & Peruvian pepper (Schinus molle) & $\mathrm{SFE} / \mathrm{CO}_{2}$ & [237] \\
\hline Limonene & Oregano (Origanum vulgare) & $\mathrm{SFE} / \mathrm{CO}_{2}$ & {$[218,255,256]$} \\
\hline Limonene & Lemon $($ Citrus $\times$ limon $)$ & $\mathrm{SFE} / \mathrm{CO}_{2}$ & [219] \\
\hline Limonene & Lemongrass (Cymbopogon citrates) & $\mathrm{HD} ; \mathrm{SFE} / \mathrm{CO}_{2}$ & {$[271]$} \\
\hline Limonene & Indian dill (Anethum sowa) & $\mathrm{HD} ; \mathrm{SFE} / \mathrm{CO}_{2}$ & [259] \\
\hline Limonene & Red clover (Trifolium pratense) & $\mathrm{SFE} / \mathrm{CO}_{2}$ & {$[255,284]$} \\
\hline Limonene & Carqueja (Baccharis genistelloides) & $\mathrm{SFE} / \mathrm{CO}_{2}$ & {$[272]$} \\
\hline Linalool & $\begin{array}{l}\text { Lavandin super } \\
\text { (Lavandula } x \text { intermedia Super) }\end{array}$ & $\mathrm{SFE} / \mathrm{CO}_{2}$ & [217] \\
\hline Linalool & $\begin{array}{l}\text { Levandin grosso } \\
\text { (Lavandula x intermedia Grosso) }\end{array}$ & $\mathrm{SFE} / \mathrm{CO}_{2}$ & [217] \\
\hline Linalool & $\begin{array}{l}\text { Lavandin abrial } \\
\text { (Lavandula } x \text { intermedia Abrial) }\end{array}$ & $\mathrm{SFE} / \mathrm{CO}_{2}$ & [217] \\
\hline
\end{tabular}




\begin{tabular}{|c|c|c|c|}
\hline Linalool & French lavender (Lavandula stoechas) & $\mathrm{SFE} / \mathrm{CO}_{2}$ & [209] \\
\hline Linalool & Sweet bay (Laurus nobilis L.) & $\mathrm{SFE} / \mathrm{CO}_{2}$ & {$[228,229]$} \\
\hline Linalool & (Thymus lotocephalus G. Lopez \& R. Morales) & $\mathrm{SFE} / \mathrm{CO}_{2}$ & [239] \\
\hline Linalool & Carqueja (Baccharis genistelloides) & $\mathrm{SFE} / \mathrm{CO}_{2}$ & [272] \\
\hline Linalool & French lavender (Lavandula stoechas) & $\mathrm{SFE} / \mathrm{CO}_{2}$ & [220] \\
\hline Linalool & Lavender (Lavandula angustifolia) & $\mathrm{SFE} / \mathrm{CO}_{2}$ & {$[241,242]$} \\
\hline Linalyl acetate & Lavender (Lavandula angustifolia) & $\mathrm{SFE} / \mathrm{CO}_{2}$ & {$[241,242]$} \\
\hline Menthol & Peppermint (Metha piperita) & $\mathrm{SFE} / \mathrm{CO}_{2}$ & [223] \\
\hline Menthol & Peppermint (Mentha piperita) & $\mathrm{SFE} / \mathrm{CO}_{2}$ & {$[189,234,285$} \\
\hline Menthone & Peppermint (Mentha piperita) & $\mathrm{SFE} / \mathrm{CO}_{2}$ & {$[189,234,285$} \\
\hline Myrcene & $\begin{array}{l}\text { Common juniper } \\
\text { (Juniperus communis L.) }\end{array}$ & $\mathrm{SFE} / \mathrm{CO}_{2}$ & [286] \\
\hline Myrcene & Sage (Salvia officinalis L.) & $\mathrm{HD} ; \mathrm{SFE} / \mathrm{CO}_{2}$ & {$[223-226]$} \\
\hline Myrcene & Lemongrass (Cymbopogon citrates) & $\mathrm{HD} ; \mathrm{SFE} / \mathrm{CO}_{2}$ & [271] \\
\hline Myrcene & Surinam cherry (Eugenia uniflora) & $\mathrm{SFE} / \mathrm{CO}_{2}$ & [51] \\
\hline Myrtenol & French lavender (Lavandula stoechas) & $\mathrm{HD} ; \mathrm{SubWE} / \mathrm{H}_{2} \mathrm{O}$ & [209] \\
\hline Myrtenol & Lavender (Lavandula viridis) & $\mathrm{HD} ; \mathrm{SFE} / \mathrm{CO}_{2}$ & [243] \\
\hline N.D. & Black pepper (Piper nigrum) & $\mathrm{SFE} / \mathrm{CO}_{2}$ & {$[287,288]$} \\
\hline N.D. & Orange & $\mathrm{SFE} / \mathrm{CO}_{2}$; ethane & [221] \\
\hline N.D. & Abise (Pimpinella anisum) & $\mathrm{HD} ; \mathrm{SFE} / \mathrm{CO}_{2}$ & [289] \\
\hline N.D. & Silver vine (Actinida polygama) & $\mathrm{SFE} / \mathrm{CO}_{2}$ & [290] \\
\hline Nanocosane & Abajeru (Chrysobalanus icaco) & $\mathrm{SE} ; \mathrm{SFE} /$ ethanol; $\mathrm{CO}_{2}$ & {$[280]$} \\
\hline Nepetalactone & Annual herbs (Nepeta persica) & $\begin{array}{l}\mathrm{HD} ; \mathrm{SFE} / \mathrm{CO}_{2}+ \\
\text { methanol }\end{array}$ & [278] \\
\hline Nerol & $\begin{array}{l}\text { Moldavian dragonhead } \\
\text { (Dracocophalum moldavica L.) }\end{array}$ & $\mathrm{SFE} / \mathrm{CO}_{2}$ & [210] \\
\hline Ocimene & Oregano (Origanum vulgare) & $\mathrm{SFE} / \mathrm{CO}_{2}$ & {$[218,255,256]$} \\
\hline Ocimene & Sage (Salvia officinalis L.) & $\mathrm{HD} ; \mathrm{SFE} / \mathrm{CO}_{2}$ & {$[223-226]$} \\
\hline Ocimene & Sweet bay (Laurus nobilis L.) & $\mathrm{SFE} / \mathrm{CO}_{2}$ & {$[228,229]$} \\
\hline Ocimene & Carqueja (Baccharis genistelloides) & $\mathrm{SFE} / \mathrm{CO}_{2}$ & {$[272]$} \\
\hline Ocimene & Corn parsley (Ridolfia segetum) & $\mathrm{SFE} / \mathrm{CO}_{2}$ & [291] \\
\hline Octanol acetate & Boswellia (Boswellia carterii) & $\mathrm{HD} ; \mathrm{SFE} / \mathrm{CO}_{2}$ & [281] \\
\hline P-Cymene & Lippia origanoides & $\mathrm{SFE} / \mathrm{CO}_{2}$ & [214] \\
\hline P-Cymene & Khoa (Satureja boliviana) & $\mathrm{SFE} / \mathrm{CO}_{2}+$ ethanol & {$[262]$} \\
\hline P-Cymene & Black cumin (Nigella sativa L.) & $\mathrm{MAE} ; \mathrm{SFE} / \mathrm{CO}_{2}$ & {$[202]$} \\
\hline Phyllocladene & Boswellia (Boswellia carterii) & $\mathrm{HD} ; \mathrm{SFE} / \mathrm{CO}_{2}$ & [281] \\
\hline Rosmanol & Rosemary (Rosmarinus officinalis L.) & $\mathrm{SFE} / \mathrm{CO}_{2}+$ ethanol & $\begin{array}{l}{[197,212,225,} \\
244-250]\end{array}$ \\
\hline Rosmanol & Rosemary (Rosmarinus officinalis L.) & $\mathrm{SubWE} / \mathrm{H}_{2} \mathrm{O}$ & {$[184,194]$} \\
\hline Rosmanol & Rosemary (Rosmarinus officinalis L.) & $\mathrm{SFE} / \mathrm{CO}_{2}$ & {$[251,253]$} \\
\hline Rosmanol & Rosemary (Rosmarinus officinalis L.) & $\begin{array}{l}\text { SFE; PLE } / \mathrm{CO}_{2} \text {; water, } \\
\text { ethanol }\end{array}$ & [254] \\
\hline Rosmarinic acid & Rosemary (Rosmarinus officinalis L.) & $\mathrm{SFE} / \mathrm{CO}_{2}+$ ethanol & $\begin{array}{l}{[197,212,225,} \\
244-250]\end{array}$ \\
\hline
\end{tabular}




\begin{tabular}{|c|c|c|c|}
\hline Rosmarinic acid & Rosemary (Rosmarinus officinalis L.) & $\mathrm{SubWE} / \mathrm{H}_{2} \mathrm{O}$ & {$[184,194]$} \\
\hline Rosmarinic acid & Rosemary (Rosmarinus officinalis L.) & $\mathrm{SFE} / \mathrm{CO}_{2}$ & {$[251,253]$} \\
\hline Rosmarinic acid & Rosemary (Rosmarinus officinalis L.) & $\begin{array}{l}\text { SFE; PLE } / \mathrm{CO}_{2} \text {; water, } \\
\text { ethanol }\end{array}$ & [254] \\
\hline Sabinene & Common juniper (Juniperus communis L.) & SFE & [286] \\
\hline Sabinene & Sweet bay (Laurus nobilis L.) & $\mathrm{SFE} / \mathrm{CO}_{2}$ & {$[228,229]$} \\
\hline Sabinene & Hyssop (Hyssopus officinalis). & $\mathrm{SFE} / \mathrm{CO}_{2}+$ methanol & [292] \\
\hline Sabinene & Seseli (Seseli bocconi) & $\mathrm{SFE} / \mathrm{CO}_{2}$ & [293] \\
\hline Sabinene & Carrot (Daucus carota) & $\mathrm{SFE} / \mathrm{CO}_{2}$ & [273] \\
\hline Sabinene & Coral ginger (Zingber corallinum) & $\mathrm{HD} ; \mathrm{SFE} / \mathrm{CO}_{2}$ & [294] \\
\hline Salinene & Curry plant (Helichrysum italicum) & $\mathrm{HD} ; \mathrm{SFE} / \mathrm{CO}_{2}$ & [261] \\
\hline Santalol & Indian sandalwood (Santal album) & $\mathrm{HD} ; \mathrm{SFE} / \mathrm{CO}_{2}$ & [281] \\
\hline Scopoletin & Sweet wormwood (Artemisia annua) & $\mathrm{SFE} / \mathrm{CO}_{2}+$ ethanol & [236] \\
\hline Sesquiterpenes & Sweetleaf (Stevia rebaudiana) & $\mathrm{SFE} / \mathrm{CO}_{2}$ & [295] \\
\hline Terpinen-4-ol & Coral ginger (Zingber corallinum) & $\mathrm{HD} ; \mathrm{SFE} / \mathrm{CO}_{2}$ & [294] \\
\hline Thymol & Oregano (Origanum vulgare) & $\mathrm{SFE} / \mathrm{CO}_{2}$ & {$[218,255,256]$} \\
\hline Thymol & Oregano (Origanum vulgare) & SubWE/H2O & [263] \\
\hline Thymol & Summer savory (Satureja hortensis) & $\begin{array}{l}\mathrm{HD}, \mathrm{SFE} / \mathrm{CO}_{2}+ \\
\text { methanol }\end{array}$ & [257] \\
\hline Thymol & Khoa (Satureja boliviana) & $\mathrm{SFE} / \mathrm{CO}_{2}+$ ethanol & [262] \\
\hline Thymol & Winter savory (Satureja montana) & $\mathrm{SFE} / \mathrm{CO}_{2}, \mathrm{HD}$ & {$[258]$} \\
\hline Thymoquinone & Black cumin (Nigella sativa L.) & $\mathrm{MAE} ; \mathrm{SFE} / \mathrm{CO}_{2}$ & [202] \\
\hline Valerenic acid & Sage (Salvia officinalis L.) & $\mathrm{HD} ; \mathrm{SFE} / \mathrm{CO}_{2}$ & {$[223-226]$} \\
\hline Valerenic acid & $\begin{array}{l}\text { Cotton lavender } \\
\text { (Santolina chamaecyparissus) }\end{array}$ & $\mathrm{HD} ; \mathrm{SFE} / \mathrm{CO}_{2}$ & [227] \\
\hline Valerenic acid & Valerian (Valeriana officinalis) & $\mathrm{SFE} / \mathrm{CO}_{2}$ & {$[296,297]$} \\
\hline Verbenone & Rosemary (Rosmarinus officinalis L.) & $\mathrm{SFE} / \mathrm{CO}_{2}+$ ethanol & $\begin{array}{l}{[164,205,225,} \\
249]\end{array}$ \\
\hline Verbenone & Lavender (Lavandula viridis) & $\mathrm{HD} ; \mathrm{SFE} / \mathrm{CO}_{2}$ & [243] \\
\hline$\alpha$-bergamotol & Indian sandalwood (Santal album) & $\mathrm{HD} ; \mathrm{SFE} / \mathrm{CO}_{2}$ & [281] \\
\hline$\alpha$-cyperone & Surinam cherry (Eugenia uniflora) & $\mathrm{SFE} / \mathrm{CO}_{2}$ & [51] \\
\hline$\alpha$-cyperone & Purple nutsedge (Cyperus rotundus) & $\mathrm{SFE} / \mathrm{CO}_{2}$ & {$[277]$} \\
\hline$\alpha$-calacorene & (Diplotaenia cachrydifolia) & $\mathrm{SFE} / \mathrm{CO}_{2}+$ ethanol & [274] \\
\hline$\alpha$-humulene & Carrot (Daucus carota) & $\mathrm{SFE} / \mathrm{CO}_{2}$ & [273] \\
\hline$\alpha$-pinnene & Borage (Borago officinalis) & $\begin{array}{l}\mathrm{HD} ; \mathrm{SFE} / \mathrm{CO}_{2}+ \\
\text { methanol }\end{array}$ & [240] \\
\hline$\alpha$-pinnene & Swamp mallet (Eucalyptus spathulata) & $\mathrm{HD} ; \mathrm{SFE} / \mathrm{CO}_{2}$ & [222] \\
\hline$\alpha$-pinnene & Coolabah (Eucalyptus microtheca) & $\mathrm{HD} ; \mathrm{SFE} / \mathrm{CO}_{2}$ & [222] \\
\hline$\alpha$-pinnene & Oregano (Origanum vulgare) & $\mathrm{SFE} / \mathrm{CO}_{2}$ & {$[218,255,256]$} \\
\hline$\alpha$-pinnene & Rosemary (Rosmarinus officinalis L.) & $\mathrm{SFE} / \mathrm{CO}_{2}+$ ethanol & {$[225,244]$} \\
\hline$\alpha$-pinnene & Cnidium monnieri & $\mathrm{SFE} / \mathrm{CO}_{2}$ & [164] \\
\hline$\alpha$-pinnene & Common juniper (Juniperus communis L.) & $\mathrm{SFE} / \mathrm{CO}_{2}$ & [286] \\
\hline$\alpha$-pinnene & Sage (Salvia officinalis L.) & $\mathrm{HD} ; \mathrm{SFE} / \mathrm{CO}_{2}$ & {$[223-226]$} \\
\hline$\alpha$-pinnene & Sweet bay (Laurus nobilis L.) & $\mathrm{SFE} / \mathrm{CO}_{2}$ & {$[228,229]$} \\
\hline
\end{tabular}




\begin{tabular}{|c|c|c|c|}
\hline$\alpha$-pinnene & Red clover (Trifolium pratense) & $\mathrm{SFE} / \mathrm{CO}_{2}$ & {$[255,284]$} \\
\hline$\alpha$-pinnene & Carrot (Daucus carota) & $\mathrm{SFE} / \mathrm{CO}_{2}$ & {$[273]$} \\
\hline$\alpha$-pinnene & Sweet gale (Myrica gale L) & $\mathrm{SFE} / \mathrm{CO}_{2}$ & {$[233]$} \\
\hline$\alpha$-selinene & Guava (Psidium guajava) & $\begin{array}{l}\mathrm{SFE} / \mathrm{CO}_{2}+\text { ethanol, }+ \\
\text { isopropanol }\end{array}$ & [298] \\
\hline$\alpha$-thujene & Black cumin (Nigella sativa L.) & $\mathrm{MAE} ; \mathrm{SFE} / \mathrm{CO}_{2}$ & {$[202]$} \\
\hline$\alpha$-thujone & Sage (Salvia officinalis) & $\mathrm{SFE} / \mathrm{CO}_{2}$ & {$[223]$} \\
\hline$\alpha$-ylangene & Five-flavor berry (Schisandra chinensis) & $\mathrm{SFE} ; \mathrm{HD} ; \mathrm{SFE} / \mathrm{CO}_{2}$ & [215] \\
\hline$\beta$-cadinene & Saigon cinnamon (Cinnamomum loureiroi) & $\mathrm{SFE} / \mathrm{CO}_{2}$ & {$[230]$} \\
\hline$\beta$-cadinene & Marigold (Calendula officinalis) & $\mathrm{SFE} / \mathrm{CO}_{2}$ & {$[238]$} \\
\hline$\beta$-caryophyllene & Carrot (Daucus carota) & $\mathrm{SFE} / \mathrm{CO}_{2}$ & {$[273]$} \\
\hline$\beta$-caryophyllene & Spearmint (Mentha spicata) & $\begin{array}{l}\mathrm{SE} ; \mathrm{SFE} / \text { hexane, DCM, } \\
\text { ethanol; } \mathrm{CO}_{2}\end{array}$ & {$[260]$} \\
\hline$\beta$-caryophyllene & Guava (Psidium guajava) & $\begin{array}{l}\mathrm{SFE} / \mathrm{CO}_{2}+\text { ethanol, }+ \\
\text { isopropanol }\end{array}$ & [298] \\
\hline$\beta$-selinene & Guava (Psidium guajava) & $\begin{array}{l}\mathrm{SFE} / \mathrm{CO}_{2}+\text { ethanol, }+ \\
\text { isopropanol }\end{array}$ & [298] \\
\hline$\beta$-selinene & Clove basil (Ocimum gratissimum) & $\mathrm{SFE} / \mathrm{CO}_{2}$ & {$[231]$} \\
\hline$\gamma$-terpinene & Oregano (Origanum vulgare) & $\mathrm{HD} ; \mathrm{SFE} / \mathrm{CO}_{2}$ & {$[218]$} \\
\hline$\gamma$-terpinene & Oregano (Origanum vulgare) & $\mathrm{SubWE} / \mathrm{H}_{2} \mathrm{O}$ & {$[263]$} \\
\hline$\gamma$-terpinene & Cotton lavender (Santolina chamaecyparissus) & $\mathrm{HD} ; \mathrm{SFE} / \mathrm{CO}_{2}$ & {$[227]$} \\
\hline$\gamma$-terpinene & Winter savory (Satureja montana) & $\mathrm{HD} ; \mathrm{SFE} / \mathrm{CO}_{2}$ & {$[258]$} \\
\hline$\gamma$-terpinene & Summer Savory (Satureja hortensis) & $\begin{array}{l}\mathrm{HD}, \mathrm{SFE} / \mathrm{CO}_{2}+ \\
\text { methanol }\end{array}$ & {$[257]$} \\
\hline$\gamma$-terpinene & Black cumin (Bunium persicum) & $\mathrm{HD} ; \mathrm{SFE} / \mathrm{CO}_{2}$ & {$[299]$} \\
\hline$\delta$-cadinene & Madeira mahogany (Persea indica) & $\mathrm{SFE} / \mathrm{CO}_{2}+$ ethanol & {$[264]$} \\
\hline$\delta$-cadinene & Honeyherb (Lippia dulcis) & $\mathrm{HD} ; \mathrm{SFE} / \mathrm{CO}_{2}$ & {$[265]$} \\
\hline$\tau$-cadinol & Dragonhead (Dracocophalum tanguticum) & $\mathrm{SFE} / \mathrm{CO}_{2}$ & {$[267]$} \\
\hline
\end{tabular}

\subsection{Extraction of lipids and fatty acids}

In Table 5, examples of SFE application for the separation of lipids and fatty acids from plant material are listed.

There are several fatty acids or their derivates that are interesting for investigation because of their functional potential. Precursors of conjugated linoleic acid, linoleic acid, and polyunsaturated fatty acid (PUFA) are intensively produced in plants. Plants primarily produce fatty acids in order to become triglycerides, which serve as stored energy (oils) as well as components of the cell membrane, i.e., glycerophospholipids and glyceroglycolipids, which have roles similar to phospholipids in humans. Major fatty acids produced in plants are palmitic acid (16:0), oleic acid (18:1 $\omega-9)$, linoleic acid (18:2 $\omega-6)$, and $\alpha$-linolenic acid $(18: 3 \omega-3)[12]$. Among fatty acids are essential fatty acids ( $\omega-6$ and $\omega-3)$, which are very important for human health as antioxidants and because they dissolve vitamins that do not dissolve in water. Those fatty acids in most cases have to be provided to the body through nutrition because the body cannot produce them.

Besides fatty acids, there are two other types of lipids in food products: first are structured lipids and the others are diglycerides. Structured lipids are triglycerides that have undergone hydrolysis and esterification, which resulted in triglycerides with a new combination 
of fatty acids. Diglycerides have been used as emulsifying agents in manufactured food products [12].

Phospholipids compose the main part of the cell membranes and are composed of diglycerides and a phosphate group, on which another organic compound is attached, such as choline [300], serine, and ethanolamine.

Lipids are mainly isolated with organic solvents, and most of them pollute the final product. To avoid further processing in order to separate organic solvents, supercritical fluid extraction could be used.

Separation is relatively easy to achieve because of the nonpolar nature of lipids. Several works have been published where the performance of conventional solvent extraction (SE) was compared to that of SFE. Even though SE generally gives higher yields than SFE, the use of organic solvents does not provide the desired selectivity; hence, other compounds besides fatty acids are extracted [301]. In order to have a faster and more selective extraction, SFE is the alternative to use.

The most common use of SFE is in the removal of oils (edible) from plant seeds and kernels. Oil-and-fat-processing industries encounter oil acidification due to the hydrolysis of the fatty acid chains and lipid oxidation, which is caused by an enzyme, heat, or moisture. In order to avoid hydrolysis, chemical and physical deacidification methods have been used in the industry. Some processes of deacidification could be performed by SFE [179, 302, 303]. When oils are removed from a solid material, extraction is usually semicontinuous. Alternatively, if bare oils are extracted and fractioned, the process is performed continuously in countercurrent mode. Such a process was performed by Al-Darmaki et al. [304] when they extracted squalene from a palm oil fatty-acid distillate. In the first set of experiments [304], pressure was changed and temperature remained constant. Afterward, a set of experiments followed, where temperature was changed and pressure was kept constant. At the highest pressure at constant temperature, recovery of squalene was the highest, while it was the lowest at the highest temperature at constant pressure [304].

Materials that contain a lot of oil extractables can be pretreated with $\mathrm{SC}-\mathrm{CO}_{2}$ [305]. Fatty acids and triglycerides dissolve $\mathrm{CO}_{2}$ well under pressure. Meyer et al. [305] performed a $\mathrm{CO}_{2}$ pretreatment of sunflower and St. John's wort. The material was exposed to compressed and heated $\mathrm{CO}_{2}$ in a magnetic suspension balance (MSB). Absorbed $\mathrm{CO}_{2}$ caused expansion of extractables in cells; during the fast decompression and desorption, the material was pulverized, and as consequence the material's contact area became bigger, and thus faster separation was achievable. In the case when a material does not contain many lipid extractables, this phenomenon did not occure [305].

SFE can be applied for the isolation of tocopherols and phytosterols from residues of vegetable-oil processing, which are deodorized - i.e. offensive odor is removed. Such residues could be obtained after the removal of oil from sunflower [1], soybean [306-309], olive [310], corn, canola, etc.

The use of $\mathrm{SC}-\mathrm{CO}_{2}$ for the extraction of essential fatty acids from plant materials has become very attractive. Such case is the isolation of $\omega-3$ and other PUFA from plant materials rich in oils, such as flaxseed [311] or canola seed [312]. Other examples could be seen in Table 5, where applications of SFE for the isolation of fatty acids and other lipids from plant material are listed.

Not only $\mathrm{SC}-\mathrm{CO}_{2}$ could be used as a solvent for the extraction of oils and fats, but subcritical propane and supercritical sulphur hexafluoride $\left(\mathrm{SF}_{6}\right)$ could also be used as solvents in extraction processes. Ilic et al. [313] measured the phase behavior of sunflower and soybean oils in propane and $\mathrm{SF}_{6}$. 
Table 5

Application of SFE for the separation of lipid compounds from natural matter

\begin{tabular}{|c|c|c|c|}
\hline Compound(s) & Plant material (Biological name) & Methods/solvents & Reference(s) \\
\hline Campesterol & Carrot (Daucus carota) & $\mathrm{SFE} / \mathrm{CO}_{2}$ & {$[273]$} \\
\hline Campesterol & Red globe grape & $\mathrm{SFE} / \mathrm{CO}_{2}+$ ethanol & {$[314]$} \\
\hline Campesterol & Victoria globe grape & $\mathrm{SFE} / \mathrm{CO}_{2}+$ ethanol & {$[314]$} \\
\hline Cycloartenol & Carrot (Daucus carota) & $\mathrm{SFE} / \mathrm{CO}_{2}$ & {$[273]$} \\
\hline Ergosterol & Shitake (Lentinula edodes) & $\begin{array}{l}\mathrm{SE} ; \mathrm{SFE} / n \text {-hexane, DCM; } \\
\mathrm{CO}_{2}\end{array}$ & {$[315]$} \\
\hline Erucic acid & Canola (Brassica napus) & $\mathrm{SFE} / \mathrm{CO}_{2}$ & {$[307,316]$} \\
\hline Eicosenoic acid & Canola (Brassica napus) & $\mathrm{SFE} / \mathrm{CO}_{2}$ & {$[307,316]$} \\
\hline Fatty acid (unsaturated) & Cuphea (Cuphea lanceolata) & $\mathrm{SFE} / \mathrm{CO}_{2}$ & {$[317]$} \\
\hline Fatty acid (unsaturated) & Passion fruit (Passiflora) & $\mathrm{SFE} / \mathrm{CO}_{2}$ & {$[318]$} \\
\hline Fatty acid (unsaturated) & $\begin{array}{l}\text { Seabuckthorn (Hippophae } \\
\text { rhamnoides) }\end{array}$ & $\mathrm{SFE} / \mathrm{CO}_{2}$ & {$[196,319]$} \\
\hline Fatty acid (unsaturated) & $\begin{array}{l}\text { Sikkim microula (Microula } \\
\text { sikkimenisis) }\end{array}$ & $\mathrm{SFE} / \mathrm{CO}_{2}$ & {$[320]$} \\
\hline Fatty acid (unsaturated) & White pitaya (Hylocereus undatus) & $\mathrm{SE} ; \mathrm{MAE} ; \mathrm{AEE} ; \mathrm{SFE} / \mathrm{CO}_{2}$ & {$[321]$} \\
\hline Fatty acid (unsaturated) & Rice (Oryza sativa) & $\mathrm{SFE} / \mathrm{CO}_{2}+$ ethanol & {$[137]$} \\
\hline Fatty acid (unsaturated) & African oil palm (Elaais guinensis) & $\mathrm{SFE} / \mathrm{CO}_{2}$ & {$[322]$} \\
\hline Hexadecanal & Lotus (Nelumbo nucifera) & $\mathrm{SFE} / \mathrm{CO}_{2}$ & {$[64]$} \\
\hline Linoleic acid & Broccoli (Brassica oleracea) & $\mathrm{SFE} / \mathrm{CO}_{2}$ & {$[323]$} \\
\hline Linoleic acid & Canola (Brassica napus) & $\mathrm{SFE} ; \mathrm{SE} / \mathrm{CO}_{2} ; n$-hexane & {$[171,324]$} \\
\hline Linoleic acid & Canola (Brassica napus) & $\mathrm{SFE} / \mathrm{CO}_{2}$ & {$[307,316]$} \\
\hline Linoleic acid & Canola (Brassica napus) & $\mathrm{SFE} / \mathrm{CO}_{2}$; propane & {$[325]$} \\
\hline Linoleic acid & Canola (Brassica napus) & $\mathrm{SFE} / \mathrm{CO}_{2}+$ ethanol & {$[326]$} \\
\hline Linoleic acid & $\begin{array}{l}\text { Cantaloupe (Cucumis melo } \\
\text { var. cantalupensis) }\end{array}$ & $\mathrm{SFE} / \mathrm{CO}_{2}$ & {$[327]$} \\
\hline Linoleic acid & Cork oak (Quercus suber) & $\mathrm{SE} ; \mathrm{SFE} / n$-hexane; $\mathrm{CO}_{2}$ & {$[328]$} \\
\hline Linoleic acid & Gardenia (Gardenia jasminoides) & $\mathrm{SFE} / \mathrm{CO}_{2}$ & {$[329]$} \\
\hline Linoleic acid & Holm oak (Quercus rotundifolia) & $\mathrm{SFE} / \mathrm{CO}_{2}$ & {$[330]$} \\
\hline Linoleic acid & Holm oak (Quercus rotundifolia) & $\mathrm{SE} ; \mathrm{SFE} / n$-hexane; $\mathrm{CO}_{2}$ & {$[328]$} \\
\hline Linoleic acid & Borage (Borago officinalis) & $\mathrm{SFE} / \mathrm{CO}_{2}+$ methanol & {$[240]$} \\
\hline Linoleic acid & Kenaf (Hibiscus cannabinus) & $\mathrm{SFE} / \mathrm{CO}_{2}$ & {$[331,332]$} \\
\hline Linoleic acid & Agaricus brasiliensis & $\mathrm{SFE} / \mathrm{CO}_{2}+$ ethanol & {$[333]$} \\
\hline Linoleic acid & Olive (Olea europaea) & $\mathrm{SFE} / \mathrm{CO}_{2}$ & {$[112]$} \\
\hline Linoleic acid & Pea (Pisum sativum) & $\mathrm{SFE} / \mathrm{CO}_{2}+$ ethanol & {$[326]$} \\
\hline Linoleic acid & Rosehip (Rosa canina) & $\mathrm{SFE} / \mathrm{CO}_{2}$ & {$[78]$} \\
\hline Linoleic acid & Siam pumpkin (Cucurbita ficifolia) & $\mathrm{SFE} ; \mathrm{SE} / \mathrm{CO}_{2}$ & {$[334]$} \\
\hline Linoleic acid & Soybean (Glycine max) & $\mathrm{SFE} / \mathrm{CO}_{2}$ & {$[307,335]$} \\
\hline Linoleic acid & Soybean (Glycine max) & $\mathrm{SFE} / \mathrm{CO}_{2}+$ ethanol & {$[326]$} \\
\hline Linoleic acid & Tea (Camellia sinensis) & $\begin{array}{l}\mathrm{SFE} / \mathrm{CO}_{2}+\text { ethanol; + } \\
\text { methanol }\end{array}$ & {$[336]$} \\
\hline
\end{tabular}


Linoleic acid

Linoleic acid

Linoleic acid

Linoleic acid

Linoleic acid

Linoleic acid

Linoleic acid

Linoleic acid

Linoleic acid

Linolenic acid

Linolenic acid

Linolenic acid

Linolenic acid

Linolenic acid

Linolenic acid

Linolenic acid

Linolenic acid

Linolenic acid

Linolenic acid

Linolenic acid

Linolenic acid

Linolenic acid

Linolenic acid

Linolenic acid

N.D.

N.D.

N.D.

N.D.

N.D.

N.D.

N.D.

N.D.

Oleic acid

Oleic acid

Oleic acid

Oleic acid

Oleic acid

Oleic acid
Peach kernel (Prunus persica)

Shitake (Lentinula edodes)

Peach kernel (Prunus persica)

Prickly pear (Opuntia dillenii)

Sunflower (Helianthus annuus)

Sunflower (Helianthus annuus)

Sunflower (Helianthus annuus)

Viper's bugloss (Echium amoenum)

Tea (Camellia sinensis)

Broccoli (Brassica oleracea)

Canola (Brassica napus)

Canola (Brassica napus)

Canola (Brassica napus)

Canola (Brassica napus)

Lotus (Nelumbo nucifera Gaertn)

Olive (olea europaea)

Pea (Pisum sativum)

Prickly pear (Opuntia dillenii)

Rosehip (Rosa canina)

Soybean (Glycine max)

Soybean (Glycine max)

Tea (Camellia sinensis)

Carrot (Daucus carota)

Tea (Camellia sinensis)

Almond (Prunus dulcis)

Apricot (Prunus armeniaca)

Guava (Psidium guajava)

Okra (Hibiscus esculentus)

Pomegranate (Punica granatum)

Sesame (Sesamun indicum)

Sorghum (Sorghum)

Yelleowhorn (Xanthoceras sorbifolia)

Canola (Brassica napus)

Canola (Brassica napus)

Canola (Brassica napus)

Canola (Brassica napus)

Cantaloupe (Cucumis melo var. cantalupensis)

Cork oak (Quercus suber)
SE; HD; SFE/ethanol, water, DCM; $\mathrm{CO}_{2}$, + ethanol

$\mathrm{SE}$; SFE $/ n$-hexane, DCM;

$\mathrm{CO}_{2}$

$\mathrm{SFE} / \mathrm{CO}_{2}+$ ethanol

$\mathrm{SFE} / \mathrm{CO}_{2}$

$\mathrm{SFE} / \mathrm{CO}_{2}$; propane

$\mathrm{SFE} / \mathrm{CO}_{2}+$ ethanol

$\mathrm{SFE} / \mathrm{CO}_{2}$

$\mathrm{SFE} / \mathrm{CO}_{2}$

$\mathrm{SFE} / \mathrm{CO}_{2}+$ ethanol; + methanol

$\mathrm{SFE} / \mathrm{CO}_{2}$

$\mathrm{SFE} ; \mathrm{SE} / \mathrm{CO}_{2} ; n$-hexane

$[171,324]$

$\mathrm{SFE} / \mathrm{CO}_{2}$

$[307,316]$

$\mathrm{SFE} / \mathrm{CO}_{2}$; propane

$\mathrm{SFE} / \mathrm{CO}_{2}+$ ethanol

$\mathrm{SFE} / \mathrm{CO}_{2}$

$\mathrm{SFE} / \mathrm{CO}_{2}$

$\mathrm{SFE} / \mathrm{CO}_{2}+$ ethanol

$\mathrm{SFE} / \mathrm{CO}_{2}$

$\mathrm{SFE} / \mathrm{CO}_{2}$

$\mathrm{SFE} / \mathrm{CO}_{2}$

$[307,335]$

$\mathrm{SFE} / \mathrm{CO}_{2}+$ ethanol

$\mathrm{SFE} / \mathrm{CO}_{2}+$ ethanol; + methanol

$\mathrm{SFE} / \mathrm{CO}_{2}$

$\mathrm{SFE} / \mathrm{CO}_{2}+$ ethanol; + methanol

$\mathrm{SFE} / \mathrm{CO}_{2}$

$\mathrm{SFE} / \mathrm{CO}_{2}+$ ethanol

$[171,324]$

$[307,316]$

$\mathrm{SFE} / \mathrm{CO}_{2}$ 


\begin{tabular}{|c|c|c|c|}
\hline Oleic acid & Holm oak (Quercus rotundifolia) & $\mathrm{SE} ; \mathrm{SFE} / n$-hexane; $\mathrm{CO}_{2}$ & {$[328]$} \\
\hline Oleic acid & Kenaf (Hibiscus cannabinus) & $\mathrm{SFE} / \mathrm{CO}_{2}$ & {$[331,332]$} \\
\hline Oleic acid & Pea (Pisum sativum) & $\mathrm{SFE} / \mathrm{CO}_{2}+$ ethanol & {$[326]$} \\
\hline Oleic acid & Peach kernel (Prunus persica) & $\begin{array}{l}\mathrm{SE} \text {; HD; SFE/ethanol, } \\
\text { water, } \mathrm{DCM} ; \mathrm{CO}_{2},+ \\
\text { ethanol }\end{array}$ & [337] \\
\hline Oleic acid & Peach kernel (Prunus persica) & $\mathrm{SFE} / \mathrm{CO}_{2}+$ ethanol & {$[338]$} \\
\hline Oleic acid & Siam pumpkin (Cucurbita ficifolia) & $\mathrm{SFE} ; \mathrm{SE} / \mathrm{CO}_{2}$ & {$[334]$} \\
\hline Oleic acid & Soybean (Glycine max) & $\mathrm{SFE} / \mathrm{CO}_{2}$ & {$[307,335]$} \\
\hline Oleic acid & Soybean (Glycine max) & $\mathrm{SFE} / \mathrm{CO}_{2}+$ ethanol & {$[326]$} \\
\hline Oleic acid & Sunflower (Helianthus annuus) & $\mathrm{SFE} / \mathrm{CO}_{2}$; propane & {$[339]$} \\
\hline Oleic acid & Sunflower (Helianthus annuus) & $\mathrm{SFE} / \mathrm{CO}_{2}+$ ethanol & [326] \\
\hline Oleic acid & Sunflower (Helianthus annuus) & $\mathrm{SFE} / \mathrm{CO}_{2}$ & {$[1]$} \\
\hline Oleic acid & Tea (Camellia sinensis) & $\begin{array}{l}\mathrm{SFE} / \mathrm{CO}_{2}+\text { ethanol; + } \\
\text { methanol }\end{array}$ & {$[336]$} \\
\hline Palmitic acid & $\begin{array}{l}\text { Cantaloupe (Cucumis melo } \\
\text { var. cantalupensis) }\end{array}$ & $\mathrm{SFE} / \mathrm{CO}_{2}$ & {$[327]$} \\
\hline Palmitic acid & Borage (Borago officinalis) & $\mathrm{SFE} / \mathrm{CO}_{2}+$ methanol & {$[240]$} \\
\hline Palmitic acid & Agaricus brasiliensis & $\mathrm{SFE} / \mathrm{CO}_{2}+$ ethanol & {$[333]$} \\
\hline Palmitic acid & Gardenia (Gardenia jasminoides) & $\mathrm{SFE} / \mathrm{CO}_{2}$ & [329] \\
\hline Palmitic acid & Holm oak (Quercus rotundifolia) & $\mathrm{SFE} / \mathrm{CO}_{2}$ & {$[330]$} \\
\hline Palmitic acid & Prickly pear (Opuntia dillenii) & $\mathrm{SFE} / \mathrm{CO}_{2}$ & {$[348]$} \\
\hline Palmitic acid & Rosehip (Rosa canina) & $\mathrm{SFE} / \mathrm{CO}_{2}$ & {$[78]$} \\
\hline Palmitic acid & Sunflower (Helianthus annuus) & $\mathrm{SFE} / \mathrm{CO}_{2} ;$ propane & {$[339]$} \\
\hline Palmitic acid & Sunflower (Helianthus annuus) & $\mathrm{SFE} / \mathrm{CO}_{2}+$ ethanol & [326] \\
\hline Palmitic acid & Sunflower (Helianthus annuus) & $\mathrm{SFE} / \mathrm{CO}_{2}$ & {$[1]$} \\
\hline Palmitic acid & Siam pumpkin (Cucurbita ficifolia) & $\mathrm{SFE} ; \mathrm{SFE} / \mathrm{CO}_{2}$ & {$[334]$} \\
\hline Palmitic acid & Tea (Camellia sinensis) & $\begin{array}{l}\mathrm{SFE} / \mathrm{CO}_{2}+\text { ethanol; + } \\
\text { methanol }\end{array}$ & {$[336]$} \\
\hline Palmitic acid & Broccoli (Brassica oleracea) & $\mathrm{SFE} / \mathrm{CO}_{2}$ & {$[323]$} \\
\hline Palmitic acid & Shitake (Lentinula edodes) & $\begin{array}{l}\mathrm{SE} ; \mathrm{SFE} / n \text {-hexane, DCM; } \\
\mathrm{CO}_{2}\end{array}$ & [315] \\
\hline Phytosterol & Polygala (Polygala cyparissias) & $\begin{array}{l}\mathrm{SE} ; \mathrm{SFE} / n \text {-hexane, DCM; } \\
\mathrm{CO}_{2}\end{array}$ & [349] \\
\hline Phytosterol & African oil palm (Elaais guinensis) & $\mathrm{SFE} / \mathrm{CO}_{2}$ & {$[322]$} \\
\hline Phytosterol & Pumkin (Cucurbita pepo) & $\mathrm{SFE} / \mathrm{CO}_{2}$ & {$[350]$} \\
\hline Phytosterol esters & Soybean (Glycine max) & $\mathrm{SFE} / \mathrm{CO}_{2}$ & {$[306,309,335]$} \\
\hline PUFA & Canola (Brassica napus) & $\mathrm{SFE} / \mathrm{CO}_{2}$ & [312] \\
\hline PUFA & Common walnut (Juglans regia) & $\mathrm{SFE} / \mathrm{CO}_{2}$ & {$[351]$} \\
\hline PUFA & Grape & $\mathrm{SFE} / \mathrm{CO}_{2}$; propane & {$[352]$} \\
\hline PUFA & $\begin{array}{l}\text { Microalgae (Schizochytrium } \\
\text { limacinum) }\end{array}$ & $\mathrm{SFE} / \mathrm{CO}_{2}+$ ethanol & [353] \\
\hline PUFA & Viper's bugloss (Echium amoenum) & $\mathrm{SFE} / \mathrm{CO}_{2}$ & {$[340]$} \\
\hline PUFA & Hemp (Cannabis sativa L.) & $\mathrm{SE} ; \mathrm{SFE} / \mathrm{CO}_{2}$ & {$[354,355]$} \\
\hline Spilanthol & Jambu (Spilanthes acmella) & $\mathrm{SFE} / \mathrm{CO}_{2}$ & {$[356]$} \\
\hline
\end{tabular}




\begin{tabular}{|c|c|c|c|}
\hline Squalene & African oil palm (Elaais guinensis) & $\mathrm{SFE} / \mathrm{CO}_{2}$ & {$[322]$} \\
\hline Squalene & Canola (Brassica napus) & $\mathrm{SFE} / \mathrm{CO}_{2}$ & {$[307]$} \\
\hline Squalene & Olive (olea europaea) & $\mathrm{SFE} / \mathrm{CO}_{2}$ & [310] \\
\hline Squalene & Lotus (Nelumbo nucifera Gaertn) & $\mathrm{SFE} / \mathrm{CO}_{2}$ & {$[54]$} \\
\hline Squalene & $\begin{array}{l}\text { Purple amaranthus (Amaranthus } \\
\text { paniculatus) }\end{array}$ & $\mathrm{SFE} / \mathrm{CO}_{2}$ & [357] \\
\hline Stearic acid & Rosehip (Rosa canina) & $\mathrm{SFE} / \mathrm{CO}_{2}$ & {$[78]$} \\
\hline Stearic acid & Tea (Camellia sinensis) & $\begin{array}{l}\mathrm{SFE} / \mathrm{CO}_{2}+\text { ethanol; + } \\
\text { methanol }\end{array}$ & {$[336]$} \\
\hline Stearic acid & Tea (Camellia sinensis) & $\begin{array}{l}\mathrm{SFE} / \mathrm{CO}_{2}+\text { ethanol; }+ \\
\text { methanol }\end{array}$ & {$[336]$} \\
\hline Stearic acid & $\begin{array}{l}\text { Cantaloupe (Cucumis melo } \\
\text { var. cantalupensis) }\end{array}$ & $\mathrm{SFE} / \mathrm{CO}_{2}$ & {$[327]$} \\
\hline Sterols & Canola (Brassica napus) & $\mathrm{SFE} / \mathrm{CO}_{2}$ & {$[307]$} \\
\hline Sterols & Rice (Oryza sativa) & $\mathrm{SFE} / \mathrm{CO}_{2}$ & {$[179-181,358]$} \\
\hline Stigmasterol & Red globe grape & $\mathrm{SFE} / \mathrm{CO}_{2}+$ ethanol & [314] \\
\hline Stigmasterol & Victoria globe grape & $\mathrm{SFE} / \mathrm{CO}_{2}+$ ethanol & [314] \\
\hline Stigmasterol & Carrot (Daucus carota) & $\mathrm{SFE} / \mathrm{CO}_{2}$ & [273] \\
\hline Stigmasterol & Lotus (Nelumbo nucifera Gaertn) & $\mathrm{SFE} / \mathrm{CO}_{2}$ & [54] \\
\hline Tocopherols & Seabuckthorn (Hippophae rhamnoids ) & $\mathrm{SFE} / \mathrm{CO}_{2}$ & {$[28]$} \\
\hline Tocopherols & Common walnut (Juglans regia) & $\mathrm{SFE} / \mathrm{CO}_{2}$ & {$[351]$} \\
\hline Tocopherols & Kenaf (Hibiscus cannabinus) & $\mathrm{SFE} / \mathrm{CO}_{2}$ & {$[331,332]$} \\
\hline Tocopherols & Olive (olea europaea) & $\mathrm{SFE} / \mathrm{CO}_{2}$ & {$[112]$} \\
\hline Tocopherols & Canola (Brassica napus) & $\mathrm{SFE} / \mathrm{CO}_{2}$ & {$[307]$} \\
\hline Tocopherols & Gardenia (Gardenia jasminoides) & $\mathrm{SFE} / \mathrm{CO}_{2}$ & [329] \\
\hline Tocopherols & Lotus (Nelumbo nucifera) & $\mathrm{SFE} / \mathrm{CO}_{2}$ & {$[64]$} \\
\hline Tocopherols & Rice (Oryza sativa.) & $\mathrm{SFE} / \mathrm{CO}_{2}$ & {$[179-181,358]$} \\
\hline Tocopherols & Soybean (Glycine max) & $\mathrm{SFE} / \mathrm{CO}_{2}$ & {$[307,335]$} \\
\hline Tocopherols & Sunflower (Helianthus annuus) & $\mathrm{SFE} / \mathrm{CO}_{2}$ & [1] \\
\hline Tocopherols & Red globe grape & $\mathrm{SFE} / \mathrm{CO}_{2}+$ ethanol & [314] \\
\hline Tocopherols & Victoria globe grape & $\mathrm{SFE} / \mathrm{CO}_{2}+$ ethanol & {$[314]$} \\
\hline Tocopherols & $\begin{array}{l}\text { Seabuckthorn (Hippophae rhamnoides } \\
\text { L.) }\end{array}$ & $\mathrm{SFE} / \mathrm{CO}_{2}+$ methanol & [29] \\
\hline Tocotriols & Rice (Oryza sativa) & $\mathrm{SFE} / \mathrm{CO}_{2}$ & {$[179-181,358]$} \\
\hline Triglycerides & Agarwood (Aquilaria crassna) & $\mathrm{SFE} / \mathrm{CO}_{2}$ & {$[359]$} \\
\hline Triglycerides & Cork oak (Quercus suber) & $\mathrm{SE} ; \mathrm{SFE} / n$-hexane; $\mathrm{CO}_{2}$ & {$[328]$} \\
\hline Triglycerides & Crude palm oil & $\mathrm{SFE} / \mathrm{CO}_{2}$ & {$[360]$} \\
\hline Triglycerides & Holm oak (Quercus rotundifolia) & $\mathrm{SE} ; \mathrm{SFE} / n$-hexane; $\mathrm{CO}_{2}$ & {$[328]$} \\
\hline Triglycerides & Barbados nut (Jatropha curcas) & $\mathrm{SFE} / \mathrm{CO}_{2}$ & {$[361]$} \\
\hline Triglycerides & Jelly fig (Ficus awkeotsang M.) & $\mathrm{SFE} / \mathrm{CO}_{2}$ & {$[362]$} \\
\hline Triterpenes & Polygala (Polygala cyparissias) & $\begin{array}{l}\mathrm{SE} ; \mathrm{SFE} / n \text {-hexane, DCM; } \\
\mathrm{CO}_{2}\end{array}$ & [349] \\
\hline Triterpenes & $\begin{array}{l}\text { Tasmanian blue gum (Eucalyptus } \\
\text { globulus) }\end{array}$ & $\mathrm{SFE} / \mathrm{CO}_{2}$ & {$[363]$} \\
\hline
\end{tabular}




\begin{tabular}{|c|c|c|c|}
\hline Triterpenoid & $\begin{array}{l}\text { Tasmanian blue gum (Eucalyptus } \\
\text { globulus) }\end{array}$ & $\mathrm{SFE} / \mathrm{CO}_{2}+$ ethanol & {$[364]$} \\
\hline Vitamin E & Grape* & PLE & {$[365]$} \\
\hline Vitamin E & Soybean (Glycine max) & $\mathrm{SFE} / \mathrm{CO}_{2}$ & {$[308,335]$} \\
\hline$\alpha$-linolenic acid & Chia (Salvia hispanica) & $\mathrm{SFE} / \mathrm{CO}_{2}$ & {$[366,367]$} \\
\hline$\alpha$-linolenic acid & Jelly fig (Ficus awkeotsang M.) & $\mathrm{SFE} / \mathrm{CO}_{2}$ & {$[362]$} \\
\hline$\alpha$-linolenic acid & Flax (Linum usitatissimum) & $\mathrm{SFE} / \mathrm{CO}_{2}$ & {$[311]$} \\
\hline$\alpha$-tocopherol & African oil palm (Elaais guinensis) & $\mathrm{SFE} / \mathrm{CO}_{2}$ & {$[322]$} \\
\hline$\alpha$-tocopherol & Almond (Prunus dulcis) & $\mathrm{SFE} / \mathrm{CO}_{2}$ & {$[368]$} \\
\hline$\alpha$-tocopherol & Sunflower (Helianthus annuus) & $\mathrm{SFE} / \mathrm{CO}_{2}$; propane & {$[339]$} \\
\hline$\alpha$-tocopherol & Red pepper (Capsicum annum L.) & $\mathrm{SFE} / \mathrm{CO}_{2}$ & {$[62]$} \\
\hline$\beta$-sitosterol & Kenaf (Hibiscus cannabinus) & $\mathrm{SFE} / \mathrm{CO}_{2}$ & {$[331,332]$} \\
\hline$\beta$-sitosterol & Laquat (Eribotrya japonica) & $\mathrm{SFE} / \mathrm{CO}_{2}$ & {$[369]$} \\
\hline$\beta$-sitosterol & Black alder (Alnus glutinosa) & $\mathrm{SFE} / \mathrm{CO}_{2}+$ ethanol & {$[370]$} \\
\hline$\beta$-sitosterol & Lotus (Nelumbo nucifera Gaertn) & $\mathrm{SFE} / \mathrm{CO}_{2}$ & {$[54]$} \\
\hline$\beta$-sitosterol & Carrot (Daucus carota) & $\mathrm{SFE} / \mathrm{CO}_{2}$ & {$[273]$} \\
\hline$\beta$-sitosterol & Red globe grape & $\mathrm{SFE} / \mathrm{CO}_{2}+$ ethanol & {$[314]$} \\
\hline$\beta$-sitosterol & Victoria globe grape & $\mathrm{SFE} / \mathrm{CO}_{2}+$ ethanol & {$[314]$} \\
\hline$\gamma$-linolenic acid & Viper's bugloss (Echium amoenum) & $\mathrm{SFE} / \mathrm{CO}_{2}$ & {$[340]$} \\
\hline$\gamma$-linolenic acid & Borage (Borago officinalis) & $\mathrm{SFE} / \mathrm{CO}_{2}+$ methanol & {$[240]$} \\
\hline$\gamma$-linolenic acid & Chia (Salvia hispanica) & $\mathrm{SFE} / \mathrm{CO}_{2}$ & {$[366,367]$} \\
\hline$\gamma$-linolenic acid & Flax (Linum usitatissimum) & $\mathrm{SFE} / \mathrm{CO}_{2}$ & {$[311]$} \\
\hline$\gamma$-linolenic acid & Rice (Oryza sativa) & $\mathrm{SFE} / \mathrm{CO}_{2}+$ ethanol & {$[137]$} \\
\hline$\gamma$-linolenic acid & Cyanobacteria (Arthrospira maxima) & $\mathrm{SFE} / \mathrm{CO}_{2}$ & {$[27]$} \\
\hline$\gamma$-oryzanol & Rice (Oryza sativa) & $\mathrm{SFE} / \mathrm{CO}_{2}$ & {$[179-181,358]$} \\
\hline
\end{tabular}

\subsection{Alkaloids and other bioactive phytochemicals}

In previous sections, nutraceuticals were discussed. Nutraceuticals improve food properties (flavor and taste - essential oils, color carotenoids), and others act in preventing and treating diseases (antioxidants - carotenoids, phenolic compounds, essential oils etc.). Besides nutraceuticals, some other phytochemicals could also be extracted from plants. Even though a lot of nutraceuticals extracted with SFE act as antioxidants, they also provide other health-beneficial properties - anti-inflammatory, antimicrobial, cancer prevention, etc.

Garcia-Risco et al. [371] studied SFE of thymol, which poses antioxidant, analgesic (relieve pain), and anti-inflammatory properties. The extract was further fractionated with SFC in order to achieve a better separation.

As mentioned before, SFE could serve for a sample preparation. Pretreated samples are suitable for further preparation or analysis $[372,373]$.

Alkaloids can be separated with SFE as well. The most common application of SFE for theisolation ofalkaloids is the removalofcaffeine from coffee beans and tea leaves [374-379]. The process is called decaffeination and is one of the first SFE processes that were developed at an industrial scale. The product is decaffeinated coffee and tea [378]. There are also some other alkaloids with health-beneficial properties, such as boldine [208], sinomenine [380], indole 
[381, 382], purine [383], tetrahydropalmatine [384], nicotine [385], isoquinolines [386], etc. [387] investigated safranol extraction with supercritical $\mathrm{CO}_{2}$. Safranol exhibits antioxidant properties, acts against cancer cells, and serves as an antidepressant.

SFE of bioactive compounds from plant materials was also used for the recovery of evodiamine and rutacarpine from the Evodia rutacarpa [388] fruit, hyperforin and adhyperforin from Hypericum perforatum $L$. [389], and solanosol from tobacco leaves [390].

\section{CONCLUSIONS}

In the present work, recent investigations of SFE and its applications are presented. Most of the researches were performed on a laboratory scale. These researches give insight into important properties of SFE processes and provide the data required for a potential scale up to a preparative or even industrial scale. This paper not only shows properties of SFE processes but also describes the possibilities of SFE application for the separation of nutraceuticals and other bioactive compounds at an industrial scale. SFE is appropriate for the separation of high value-added products because of its high investment cost. But obtained extracts are free from organic solvents, contributing to sustainability. The future trend of SFE shall go in the direction of not only the extraction of certain compounds but also the purification and concentration of compounds. Therefore, other separation processes could be coupled with SFE. Among them is SFC, where the purification of several compounds is possible. To implement SFE in the industry, optimization studies are required in order to reduce operating costs. Hence, proper operating parameters should be selected using different optimization methods, such as response surface methodology. To reduce operating costs even more, the recirculation of solvent should be applied. Vessels where the extract would be collected should be under pressure to reduce the amount of energy required for renewed pressurization. Further investigations of the SFE process should include the application of SFE for industrial purposes, meaning coupling with other separation processes such as SFC and micronization processes, in order to purify and concentrate and to obtain smaller particles, which could be used effectively in the food or pharmaceutical industry.

Even though many investigations were done in this field, many possibilities of SFE application from plant material could be discovered in the future.

Acknowledgments. This work was sponsored by the Slovenian Research Agency (ARRS), Program No. P2-0046.

\section{ABBREVIATIONS}

\begin{tabular}{|c|c|}
\hline SFE & Supercritical fluid extraction \\
\hline RSM & Response surface methodology \\
\hline DMP & 2,2-dimethoxypropane \\
\hline HPLC & $\begin{array}{l}\text { High-performance liquid } \\
\text { chromatography }\end{array}$ \\
\hline PHWE & Pressurized hot-water extraction \\
\hline ASE & Accelerated solvent extraction \\
\hline CC-SFE & $\begin{array}{l}\text { Countercurrent supercritical } \\
\text { fluid extraction }\end{array}$ \\
\hline RP-LC & $\begin{array}{l}\text { Reversed-phase liquid } \\
\text { extraction }\end{array}$ \\
\hline $\mathrm{C} 8$ & $\begin{array}{l}\text { Silica columns with } 8 \text {-carbon } \\
\text { chains on the surface }\end{array}$ \\
\hline $\mathrm{C} 18$ & $\begin{array}{l}\text { Silica columns with } 18 \text {-carbon } \\
\text { chains on the surface }\end{array}$ \\
\hline TLC & Thin layer chromatography \\
\hline DAD & Diode array detector \\
\hline MS & Mass spectroscopy \\
\hline ESI & Electrospray ionization detector \\
\hline ELSD & $\begin{array}{l}\text { Evaporative light-scattering } \\
\text { detector }\end{array}$ \\
\hline GC-MS & $\begin{array}{l}\text { Gas chromatography coupled } \\
\text { with mass spectroscopy }\end{array}$ \\
\hline SubWE & Subcritical water extraction \\
\hline $\mathrm{SE}$ & $\begin{array}{l}\text { Solvent extraction or Soxhlet } \\
\text { extraction }\end{array}$ \\
\hline
\end{tabular}

Maced. J. Chem. Chem. Eng. 32 (2), 183-226 (2013) 


$\begin{array}{ll}\text { CE } & \text { Capillary electrophoresis } \\ \text { HD } & \text { Hydrodistillation } \\ \text { SD } & \text { Steam distillation } \\ \text { PUFA } & \text { Polyunsaturated fatty acids } \\ \text { MSB } & \text { Magnetic suspension balance } \\ \text { SF }_{6} & \text { Sulphur hexafluoride } \\ \text { R134a } & \text { Freon 1,1,1,2-tetrafluoroethane } \\ \text { DEP } & \text { 2,2-diethoxypropane } \\ \text { TEOF } & \text { Triethyl orthoformate } \\ \text { MAE } & \text { Microwave-assisted extraction } \\ \text { AEE } & \text { Aqueous enzymatic extraction }\end{array}$

\section{REFERENCES}

[1] L. Vazquez, C. F. Torres, T. Fornari, N. Grigelmo, F. J. Senorans, G. Reglero, Supercritical fluid extract ion of minor lipids from pretreated sunflower oil deodorizer distillates, Eur. J. Lipid Sci. Technol., 108, 659-665 (2006).

[2] H. W. Wang, Y. Q. Liu, S. L. Wei, Z. J. Yan, Application of response surface methodology to optimise supercritical carbon dioxide extraction of essential oil from Cyperus rotundus Linn., Food Chem., 132, 582-587 (2012).

[3] E. J. Beckman, Supercritical and near-critical $\mathrm{CO}_{2}$ in green chemical synthesis and processing, J. Supercrit. Fluids, 28, 121-191 (2004).

[4] M. S. Diaz, E. A. Brignole, Modeling and optimization of supercritical fluid processes, J. Supercrit. Flu$i d s$, 47, 611-618 (2009).

[5] Y. E. Gorbaty, G. V. Bondarenko, The physical state of supercritical fluids, J. Supercrit. Fluids, 14, 1-8 (1998).

[6] C. G. Pereira, M. A. A. Meireles, Supercritical fluid extraction of bioactive compounds: Fundamentals, applications and economic perspectives, Food Bioprocess Tech., 3, 340-372 (2010).

[7] E. Reverchon, I. De Marco, Supercritical fluid extraction and fractionation of natural matter, J. Supercrit. Fluids, 38, 146-166 (2006). ]

[8] R. M. Weathers, D. A. Beckholt, A. L. Lavella, N. D. Danielson, Comparison of acetals as in situ modifiers for the supercritical fluid extraction of beta-carotene from paprika with carbon dioxide, J. Liq. Chromatogr. Rel. Technol., 22, 241-252 (1999).
[9] Q. Y. Lang, C. M. Wai, Supercritical fluid extraction in herbal and natural product studies - A practical review, Talanta, 53, 771-782 (2001).

[10] E. Uquiche, E. Huerta, A. Sandoval, J. M. del Valle, Effect of boldo (Peumus boldus M.) pretreatment on kinetics of supercritical $\mathrm{CO}_{2}$ extraction of essential oil, J. Food Eng., 109, 230-237 (2012).

[11] H. Sovova, R. P. Stateva, Supercritical fluid extraction from vegetable materials, Rev. Chem. Eng., 27, 79-156 (2011).

[12] R. E. C. Wildman, Handbook of nutraceuticals and functional foods, second edition, Taylor \& Francis, 2006.

[13] I. W. G. o. t. E. o. C. P. Agents, W. H. Organization, I. A. f. R. o. Cancer, Carotenoids, IARC Pess, 1998.

[14] F. Delgado-Vargas, A. R. Jimenez, O. Paredes-Lopez, Natural pigments: Carotenoids, anthocyanins, and betalains - characteristics, biosynthesis, processing, and stability, Crit. Rev. Food Sci. Nutr., 40, 173-289 (2000).

[15] T. Baysal, S. Ersus, D. A. J. Starmans, Supercritical $\mathrm{CO}_{2}$ extraction of beta-carotene and lycopene from tomato paste waste, J. Agric. Food. Chem., 48, 55075511 (2000).

[16] P. F. Leal, M. E. M. Braga, D. N. Sato, J. E. Carvalho, M. O. M. Marques, M. A. A. Meireles, Functional properties of spice extracts obtained via supercritical fluid extraction, J. Agric. Food. Chem., 51, 25202525 (2003).

[17] P. Palozza, N. I. Krinsky, Antioxidant effects of carotenoids invivo and invitro - An overview, Methods Enzymol., 213, 403-420 (1992).

[18] P. Subra, S. Castellani, P. Jestin, A. Aoufi, Extraction of beta-carotene with supercritical fluids - experiments and modelling, J. Supercrit. Fluids, 12, 261-269 (1998).

[19] I. Gamlieli-Bonshtein, E. Korin, S. Cohen, Selective separation of cis-trans geometrical isomers of betacarotene via $\mathrm{CO}_{2}$ supercritical fluid extraction, Biotechnol. Bioeng., 80, 169-174 (2002).

[20] O. Doker, U. Salgin, I. Sanal, U. Mehmetoglu, A. Calimli, Modeling of extraction of beta-carotene from apricot bagasse using supercritical $\mathrm{CO}_{2}$ in packed bed extractor, J. Supercrit. Fluids, 28, 11-19 (2004).

[21] H. L. N. Lau, Y. M. Choo, A. N. Ma, C. H. Chuah, Selective extraction of palm carotene and vitamin $\mathrm{E}$ from fresh palm-pressed mesocarp fiber (Elaeis guineensis) using supercritical $\mathrm{CO}_{2}$, J. Food Eng., 84, 289-296 (2008). 
[22] G. Vasapollo, L. Longo, L. Rescio, L. Ciurlia, Innovative supercritical $\mathrm{CO}_{2}$ extraction of lycopene from tomato in the presence of vegetable oil as co-solvent, J. Supercrit. Fluids, 29, 87-96 (2004).

[23] S. Machmudah, A. Shotipruk, M. Goto, M. Sasaki, T. Hirose, Extraction of astaxanthin from haematococcus pluvialis using supercritical $\mathrm{CO}_{2}$ and ethanol as entrainer, Ind. Eng. Chem. Res., 45, 3652-3657 (2006).

[24] B. Nobre, F. Marcelo, R. Passos, L. Beirao, A. Palavra, L. Gouveia, R. Mendes, Supercritical carbon dioxide extraction of astaxanthin and other carotenoids from the microalga Haematococcus pluvialis, Eur. Food Res. Technol., 223, 787-790 (2006).

[25] J. L. Pan, H. M. Wang, C. Y. Chen, J. S. Chang, Extraction of astaxanthin from Haematococcus pluvialis by supercritical carbon dioxide fluid with ethanol modifier, Eng. Life Sci., 12, 638-647 (2012).

[26] L. Z. Wang, B. Yang, B. L. Yan, X. C. Yao, Supercritical fluid extraction of astaxanthin from Haematococcus pluvialis and its antioxidant potential in sunflower oil, Innov. Food Sci. Emerg., 13, 120-127 (2012).

[27] R. L. Mendes, B. P. Nobre, M. T. Cardoso, A. P. Pereira, A. F. Palavra, Supercritical carbon dioxide extraction of compounds with pharmaceutical importance from microalgae, Inorg. Chim. Acta, 356, 328-334 (2003).

[28] L. D. Kagliwal, S. C. Patil, A. S. Pol, R. S. Singhal, V. B. Patravale, Separation of bioactives from seabuckthorn seeds by supercritical carbon dioxide extraction methodology through solubility parameter approach, Sep. Purif. Technol., 80, 533-540 (2011).

[29] L. D. Kagliwal, A. S. Pol, S. C. Patil, R. S. Singhal, V. B. Patravale, Antioxidant-rich extract from dehydrated seabuckthorn berries by supercritical carbon dioxide extraction, Food Bioprocess Tech., 5, 2768 2776 (2012).

[30] M. K. Roh, M. S. Uddin, B. S. Chun, Extraction of fucoxanthin and polyphenol from Undaria pinnatifida using supercritical carbon dioxide with co-solvent, Biotechnol. Bioproc. E., 13, 724-729 (2008).

[31] Y. W. Hsu, C. F. Tsai, W. K. Chen, Y. C. Ho, F. J. Lu, Determination of lutein and zeaxanthin and antioxidant capacity of supercritical carbon dioxide extract from daylily (Hemerocallis disticha), Food Chem., 129, 1813-1818 (2011).

[32] J. M. Fernandez-Sevilla, F. G. A. Fernandez, E. M. Grima, Biotechnological production of lutein and its applications, Appl. Microbiol. Biotechnol., 86, 27-40 (2010).
[33] Y. X. Gao, B. Nagy, X. Liu, B. Simandi, Q. Wang, Supercritical $\mathrm{CO}_{2}$ extraction of lutein esters from marigold (Tagetes erecta L.) enhanced by ultrasound, J. Supercrit. Fluids, 49, 345-350 (2009).

[34] W. Palumpitag, P. Prasitchoke, M. Goto, A. Shotipruk, Supercritical carbon dioxide extraction of marigold lutein fatty acid esters: Effects of cosolvents and saponification conditions, Sep. Sci. Technol., 46, 605-610 (2011).

[35] M. S. Gomez-Prieto, M. L. Ruiz del Castillo, G. Flores, G. Santa-Maria, G. P. Blanch, Application of chrastil's model to the extraction insc- $\mathrm{CO}_{2}$ of pcarotene and lutein in Mentha spicata L., J. Supercrit. Fluids, 43, 32-36 (2007).

[36] J. S. Seo, B. J. Burri, Z. J. Quan, T. R. Neidlinger, Extraction and chromatography of carotenoids from pumpkin, J. Chromatogr. A, 1073, 371-375 (2005).

[37] J. Shi, C. Yi, X. Q. Ye, S. Xue, Y. M. Jiang, Y. Ma, D. $\mathrm{H}$. Liu, Effects of supercritical $\mathrm{CO}_{2}$ fluid parameters on chemical composition and yield of carotenoids extracted from pumpkin, Lwt-Food Sci. Technol., 43, 39-44 (2010).

[38] H. Sovová, M. Sajfrtová, M. Bártlová, L. Opletal, Near-critical extraction of pigments and oleoresin from stinging nettle leaves, J. Supercrit. Fluids, 30, 213-224 (2004).

[39] D. Ruen-ngam, A. Shotipruk, P. Pavasant, S. Machmudah, M. Goto, Selective extraction of lutein from alcohol treated Chlorella vulgaris by supercritical CO${ }_{2}$, Chem. Eng. Technol., 35, 255-260 (2012).

[40] S. M. Choudhari, R. S. Singhal, Supercritical carbon dioxide extraction of lycopene from mated cultures of Blakeslea trispora NRRL 2895 and 2896, J. Food Eng., 89, 349-354 (2008).

[41] L. Ciurlia, M. Bleve, L. Rescio, Supercritical carbon dioxide co-extraction of tomatoes (Lycopersicum esculentum L.) and hazelnuts (Corylus avellana L.): A new procedure in obtaining a source of natural lycopene, J. Supercrit. Fluids, 49, 338-344 (2009).

[42] J. Shi, C. Yi, S. J. Xue, Y. M. Jiang, Y. Ma, D. Li, Effects of modifiers on the profile of lycopene extracted from tomato skins by supercritical $\mathrm{CO}_{2}, \mathrm{~J}$. Food Eng., 93, 431-436 (2009).

[43] C. Yi, J. Shi, S. J. Xue, Y. M. Jiang, D. Li, Effects of supercritical fluid extraction parameters on lycopene yield and antioxidant activity, Food Chem., 113, 1088-1094 (2009).

[44] S. Machmudah, Zakaria, S. Winardi, M. Sasaki, M. Goto, N. Kusumoto, K. Hayakawa, Lycopene extraction from tomato peel by-product containing tomato 
seed using supercritical carbon dioxide, J. Food Eng., 108, 290-296 (2012).

[45] J. A. Egydio, A. M. Moraes, P. T. V. Rosa, Supercritical fluid extraction of lycopene from tomato juice and characterization of its antioxidation activity, $J$. Supercrit. Fluids, 54, 159-164 (2010).

[46] B. P. Nobre, A. F. Palavra, F. L. P. Pessoa, R. L. Mendes, Supercritical $\mathrm{CO}_{2}$ extraction of trans-lycopene from portuguese tomato industrial waste, Food Chem., 116, 680-685 (2009).

[47] L. S. Kassama, J. Shi, G. S. Mittal, Optimization of supercritical fluid extraction of lycopene from tomato skin with central composite rotatable design model, Sep. Purif. Technol., 60, 278-284 (2008).

[48] D. Montesano, F. Fallarino, L. Cossignani, A. Bosi, M. S. Simonetti, P. Puccetti, P. Damiani, Innovative extraction procedure for obtaining high pure lycopene from tomato, Eur. Food Res. Technol., 226, 327-335 (2008)

[49] J. Pol, T. Hyotylainen, O. Ranta-Aho, M. L. Riekkola, Determination of lycopene in food by on-line SFE coupled to HPLC using a single monolithic column for trapping and separation, J. Chromatogr. A, 1052, 25-31 (2004).

[50] K. W. Kong, N. F. Rajab, K. N. Prasad, A. Ismail, M. Markom, C. P. Tan, Lycopene-rich fractions derived from pink guava by-product and their potential activity towards hydrogen peroxide-induced cellular and DNA damage, Food Chem., 123, 1142-1148 (2010).

[51] F. S. Malaman, L. A. B. Moraes, C. West, N. J. Ferreira, A. L. Oliveira, Supercritical fluid extracts from the Brazilian cherry (Eugenia uniflora L.): Relationship between the extracted compounds and the characteristic flavour intensity of the fruit, Food Chem., 124, 85-92 (2011).

[52] M. Ota, H. Watanabe, Y. Kato, M. Watanabe, Y. Sato, R. L. Smith, H. Inomata, Carotenoid production from chlorococcum littorale in photoautotrophic cultures with downstream supercritical fluid processing, $J$. Sep. Sci., 32, 2327-2335 (2009).

[53] R. Tozzi, N. Mulinacci, K. Storlikken, I. Pasquali, F. F. Vincieri, R. Bettini, Supercritical extraction of carotenoids from Rosa canina L. Hips and their formulation with beta-cyclodextrin, Aaps Pharmscitech, 9 , 693-700 (2008).

[54] X. A. Xu, J. Dong, X. F. Mu, L. P. Sun, Supercritical $\mathrm{CO}_{2}$ extraction of oil, carotenoids, squalene and sterols from lotus (Nelumbo nucifera Gaertn) bee pollen, Food Bioprod. Process., 89, 47-52 (2011).

[55] M. Takahashi, H. Watanabe, J. Kikkawa, M. Ota, M. Watanabe, Y. Sato, H. Inomata, N. Sato, Carotenoids extraction from Japanese persimmon (hachiyakaki) peels by supercritical $\mathrm{CO}_{2}$ with ethanol, Anal. Sci., 22, 1441-1447 (2006).

[56] A. Ambrogi, D. A. Cardarelli, R. Eggers, Fractional extraction of paprika using supercritical carbon dioxide and on-line determination of carotenoids, J. Food Sci., 67, 3236-3241 (2002).

[57] M. Careri, L. Furlattini, A. Mangia, M. Musci, E. Anklam, A. Theobald, C. von Holst, Supercritical fluid extraction for liquid chromatographic determination of carotenoids in Spirulina pacifica algae: A chemometric approach, J. Chromatogr. A, 912, 61-71 (2001).

[58] Y. C. Cho, J. H. Cheng, S. L. Hsu, S. E. Hong, T. M. Lee, C. M. J. Chang, Supercritical carbon dioxide anti-solvent precipitation of anti-oxidative zeaxanthin highly recovered by elution chromatography from Nannochloropsis oculata, Sep. Purif. Technol., 78, 274-280 (2011).

[59] B. C. Liau, S. E. Hong, L. P. Chang, C. T. Shen, Y. C. Li, Y. P. Wu, T. T. Jong, C. J. Shieh, S. L. Hsu, C. M. J. Chang, Separation of sight-protecting zeaxanthin from Nannochloropsis oculata by using supercritical fluids extraction coupled with elution chromatography, Sep. Purif. Technol., 78, 1-8 (2011).

[60] J. A. Mendiola, F. R. Marin, S. F. Hernandez, B. O. Arredondo, F. J. Senorans, E. Ibanez, G. Reglero, Characterization via liquid chromatography coupled to diode array detector and tandem mass spectrometry of supercritical fluid antioxidant extracts of Spirulina platensis microalga, J. Sep. Sci., 28, 1031-1038 (2005).

[61] M. G. Sajilata, M. V. Bule, P. Chavan, R. S. Singhal, M. Y. Kamat, Development of efficient supercritical carbon dioxide extraction methodology for zeaxanthin from dried biomass of Paracoccus zeaxanthini faciens, Sep. Purif. Technol., 71, 173-177 (2010).

[62] A. Romo-Hualde, A. I. Yetano-Cunchillos, C. Gonzalez-Ferrero, M. J. Saiz-Abajo, C. J. Gonzalez-Navarro, Supercritical fluid extraction and microencapsulation of bioactive compounds from red pepper (Capsicum annum L.) by-products, Food Chem., 133, 1045-1049 (2012).

[63] R. Davarnejad, K. M. Kassim, A. Zainal, S. A. Sata, Supercritical fluid extraction of beta-carotene from crude palm oil using $\mathrm{CO}_{2}$, J. Food Eng., 89, 472-478 (2008).

[64] J. L. Li, M. Zhang, T. S. Zheng, The in vitro antioxidant activity of lotus germ oil from supercritical fluid carbon dioxide extraction, Food Chem., 115, 939-944 (2009). 
[65] I. S. Sanal, A. Guvenc, U. Salgin, U. Mehmetoglu, A. Calimli, Recycling of apricot pomace by supercritical $\mathrm{CO}_{2}$ extraction, J. Supercrit. Fluids, 32, 221-230 (2004).

[66] S. J. Sarrade, G. M. Rios, M. Carles, Supercritical $\mathrm{CO}_{2}$ extraction coupled with nanofiltration separation - applications to natural products, Sep. Purif. Technol., 14, 19-25 (1998).

[67] A. N. Mustapa, Z. A. Manan, C. Y. M. Azizi, W. B. Setianto, A. K. M. Omar, Extraction of beta-carotenes from palm oil mesocarp using sub-critical R134a, Food Chem., 125, 262-267 (2011).

[68] L. Feltl, V. Pacaková, K. Stulík, K. Volka, Reliability of carotenoid analyses: A review, Current Analytical Chemistry, 1, 93-102 (2005).

[69] A. R. B. de Quiros, H. S. Costa, Analysis of carotenoids in vegetable and plasma samples: A review, $J$. Food Compos. Anal., 19, 97-111 (2006).

[70] A. I. O. Barba, M. C. Hurtado, M. C. S. Mata, V. F. Ruiz, M. L. S. de Tejada, Application of a UV-VIS detection-HPLC method for a rapid determination of lycopene and beta-carotene in vegetables, Food Chem., 95, 328-336 (2006).

[71] P. Bhosale, P. S. Bernstein, Microbial xanthophylls, Appl. Microbiol. Biotechnol., 68, 445-455 (2005).

[72] R. Edge, D. J. McGarvey, T. G. Truscott, The carotenoids as anti-oxidants - A review, J. Photoch. Photobio. B, 41, 189-200 (1997).

[73] Z. Y. Wu, S. M. Wu, X. M. Shi, Supercritical fluid extraction and determination of lutein in heterotrophically cultivated Chlorella pyrenoidosa, J. Food Process Eng., 30, 174-185 (2007).

[74] M. Guerin, M. E. Huntley, M. Olaizola, Haematococcus astaxanthin: Applications for human health and nutrition, Trends Biotechnol., 21, 210-216 (2003).

[75] R. G. Fassett, J. S. Coombes, Astaxanthin: A potential therapeutic agent in cardiovascular disease, Mar. Drugs, 9, 447-465 (2011).

[76] L. Felix-Valenzuela, I. Higuera-Ciapara, F. Goycoolea-Valencia, W. Arguelles-Monal, Supercritical $\mathrm{CO}_{2}$ /ethanol extraction of astaxanthin from blue crab (Callinectes sapidus) shell waste, J. Food Process Eng., 24, 101-112 (2001).

[77] M. Lopez, L. Arce, J. Garrido, A. Rios, M. Valcarcel, Selective extraction of astaxanthin from crustaceans by use of supercritical carbon dioxide, Talanta, 64, 726-731 (2004).

[78] S. Machmudah, Y. Kawahito, M. Sasaki, M. Goto, Supercritical $\mathrm{CO}_{2}$ extraction of rosehip seed oil: Fatty acids composition and process optimization, $\mathrm{J}$. Supercrit. Fluids, 41, 421-428 (2007).

[79] C. Quan, C. Turner, Extraction of astaxanthin from shrimp waste using pressurized hot ethanol, Chromatographia, 70, 247-251 (2009).

[80] A. Kulomaa, H. Siren, M. L. Riekkola, Identification of antioxidative compounds in plant beverages by capillary electrophoresis with the marker index technique, J. Chromatogr. A, 781, 523-532 (1997).

[81] J. Peterson, J. Dwyer, Flavonoids: Dietary occurrence and biochemical activity, Nutr. Res., 18, 19952018 (1998).

[82] M. Moraes, J. H. Y. Vilegas, F. M. Lancas, Supercritical fluid extraction of glycosilated flavonoids from passiflora leaves, Phytochem. Anal, 8, 257-260 (1997).

[83] M. Ashraf-Khorassani, L. T. Taylor, Sequential fractionation of grape seeds into oils, polyphenols, and procyanidins via a single system employing $\mathrm{CO}_{2}-$ based fluids, J. Agric. Food. Chem., 52, 2440-2444 (2004).

[84] A. Chafer, M. C. Pascual-Marti, A. Salvador, A. Berna, Supercritical fluid extraction and HPLC determination of relevant polyphenolic compounds in grape skin, J. Sep. Sci., 28, 2050-2056 (2005).

[85] L. Fiori, D. de Faveri, A. A. Casazza, P. Perego, Grape by-products: Extraction of polyphenolic compounds using supercritical $\mathrm{CO}_{2}$ and liquid organic solvent A preliminary investigation. Subproductos de la uva: Extraccion de compuestos polifenolicos usando $\mathrm{CO}_{2}$ supercritico y disolventes organicos liquidos - Una investigacion preliminar, Cyta-J. Food, 7, 163-171 (2009).

[86] M. C. Lazze, R. Pizzala, F. J. G. Pecharroman, P. G. Garnica, J. M. A. Rodriguez, N. Fabris, L. Bianchi, Grape waste extract obtained by supercritical fluid extraction contains bioactive antioxidant molecules and induces antiproliferative effects in human colon adenocarcinoma cells, J. Med. Food, 12, 561-568 (2009).

[87] R. Murga, R. Ruiz, S. Beltran, J. L. Cabezas, Extraction of natural complex phenols and tannins from grape seeds by using supercritical mixtures of carbon dioxide and alcohol, J. Agric. Food. Chem., 48, 3408-3412 (2000).

[88] B. Palenzuela, L. Arce, A. Macho, E. Munoz, A. Rios, M. Valcarcel, Bioguided extraction of polyphenols from grape marc by using an alternative supercritical-fluid extraction method based on a liquid solvent trap, Anal. Bioanal. Chem., 378, 2021-2027 (2004). 
[89] M. Pinelo, A. Arnous, A. S. Meyer, Upgrading of grape skins: Significance of plant cell-wall structural components and extraction techniques for phenol release, Trends Food Sci. Technol., 17, 579-590 (2006).

[90] M. Pinelo, A. Ruiz-Rodriguez, J. Sineiro, F. J. Senorans, G. Reglero, M. J. Nunez, Supercritical fluid and solid-liquid extraction of phenolic antioxidants from grape pomace: A comparative study, Eur. Food Res. Technol., 226, 199-205 (2007).

[91] T. Vatai, M. Škerget, Ž. Knez, Extraction of phenolic compounds from elder berry and different grape marc varieties using organic solvents and/or supercritical carbon dioxide, J. Food Eng., 90, 246-254 (2009).

[92] E. Q. Xia, G. F. Deng, Y. J. Guo, H. B. Li, Biological activities of polyphenols from grapes, Int. J. Mol. Sci., 11, 622-646 (2010).

[93] J. Yu, D. V. Dandekar, R. T. Toledo, R. K. Singh, B. S. Patil, Supercritical fluid extraction of limonoids and naringin from grapefruit (Citrus paradisi Macf.) seeds, Food Chem., 105, 1026-1031 (2007).

[94] S. Scalia, L. Giuffreda, P. Pallado, Analytical and preparative supercritical fluid extraction of chamomile flowers and its comparison with conventional methods, J. Pharm. Biomed. Anal., 21, 549-558 (1999).

[95] M. Antolovich, P. Prenzler, K. Robards, D. Ryan, Sample preparation in the determination of phenolic compounds in fruits, Analyst, 125, 989-1009 (2000).

[96] J. M. A. Araujo, M. V. Silva, J. B. P. Chaves, Supercritical fluid extraction of daidzein and genistein isoflavones from Soybean hypocotyl after hydrolysis with endogenous beta-glucosidases, Food Chem., 105, 266-272 (2007).

[97] C. Bergeron, S. Gafner, E. Clausen, D. J. Carrier, Comparison of the chemical composition of extracts from Scutellaria lateriflora using accelerated solvent extraction and supercritical fluid extraction versus standard hot water or $70 \%$ ethanol extraction, $\mathrm{J}$. $\mathrm{Ag}$ ric. Food. Chem., 53, 3076-3080 (2005).

[98] Y. Chang, B. Liu, B. Shen, Orthogonal array design for the optimization of supercritical fluid extraction of balicalin from roots of Scutellaria baicalensis Georgi, J. Sep. Sci., 30, 1568-1574 (2007).

[99] K. L. Chiu, Y. C. Cheng, J. H. Chen, C. M. J. Chang, P. W. Yang, Supercritical fluids extraction of ginkgo ginkgolides and flavonoids, J. Supercrit. Fluids, 24, 77-87 (2002).

[100] E. Dimitrieska-Stojković, Z. Zdravkovski, Supercritical fluid extraction of quercetin and rutin from Hyperici herba, J. Liq. Chromatogr. Rel. Technol., 26, 2517-2533 (2003).
[101] M. Hamburger, D. Baumann, S. Adler, Supercritical carbon dioxide extraction of selected medicinal plants - effects of high pressure and added ethanol on yield of extracted substances, Phytochem. Anal, 15, 46-54 (2004).

[102] C. S. Kaiser, H. Rompp, P. C. Schmidt, Supercritical carbon dioxide extraction of chamomile flowers: Extraction efficiency, stability, and in-line inclusion of chamomile-carbon dioxide extract in beta-cyclodextrin, Phytochem. Anal, 15, 249-256 (2004).

[103] B. Klejdus, L. Lojková, O. Lapcik, R. Koblovská, J. Moravcová, V. Kuban, Supercritical fluid extraction of isoflavones from biological samples with ultrafast high-performance liquid chromatography/mass spectrometry, J. Sep. Sci., 28, 1334-1346 (2005).

[104] K. G. Martino, D. Guyer, Supercritical fluid extraction of quercetin from onion skins, J. Food Process Eng., 27, 17-28 (2004).

[105] M. A. Rostagno, J. M. A. Araujo, D. Sandi, Supercritical fluid extraction of isoflavones from soybean flour, Food Chem., 78, 111-117 (2002).

[106] M. T. Tena, A. Rios, M. Valcarcel, Supercritical fluid extraction of $t$-resveratrol and other phenolics from a spiked solid, Fresenius J. Anal. Chem., 361, 143-148 (1998).

[107] C. Yang, Y. R. Xu, W. X. Yao, Extraction of pharmaceutical components from ginkgo biloba leaves using supercritical carbon dioxide, J. Agric. Food. Chem., 50, 846-849 (2002).

[108] J. Liu, S. Y. Lin, Z. Z. Wang, C. N. Wang, E. L. Wang, Y. Zhang, J. B. Liu, Supercritical fluid extraction of flavonoids from Maydis stigma and its nitrite-scavenging ability, Food Bioprod. Process., 89, 333-339 (2011).

[109] L. E. Laroze, B. Diaz-Reinoso, A. Moure, M. E. Zuniga, H. Dominguez, Extraction of antioxidants from several berries pressing wastes using conventional and supercritical solvents, Eur. Food Res. Technol., 231, 669-677 (2010).

[110] I. J. Seabra, M. E. M. Braga, M. T. P. Batista, H. C. de Sousa, Fractioned high pressure extraction of anthocyanins from elderberry (Sambucus nigra L.) pomace, Food Bioprocess Tech., 3, 674-683 (2010).

[111] E. Rahimi, J. M. Prado, G. Zahedi, M. A. A. Meireles, Chamomile extraction with supercritical carbon dioxide: Mathematical modeling and optimization, $J$. Supercrit. Fluids, 56, 80-88 (2011).

[112] P. Jimenez, L. Masson, A. Barriga, J. Chavez, P. Robert, Oxidative stability of oils containing olive leaf extracts obtained by pressure, supercritical and solvent-extraction, Eur. J. Lipid Sci. Technol., 113, 497-505 (2011). 
[113] M. S. Liza, R. A. Rahman, B. Mandana, S. Jinap, A. Rahmat, I. S. M. Zaidul, A. Hamid, Supercritical carbon dioxide extraction of bioactive flavonoid from Strobilanthes crispus (Pecah kaca), Food Bioprod. Process., 88, 319-326 (2010).

[114] H. Cao, M. J. Ji, H. X. Wang, Essential oil from Marchantia convoluta: Extraction and components, J. Chil. Chem. Soc., 52, 1088-1091 (2007).

[115] H. Cao, J. B. Xiao, M. Xu, Comparison of volatile components of Marchantia convoluta obtained by supercritical carbon dioxide extraction and petrol ether extraction, J. Food Compos. Anal., 20, 45-51 (2007).

[116] J. B. Xiao, J. W. Chen, M. Xu, Supercritical fluid $\mathrm{CO}_{2}$ extraction of essential oil from Marchantia convoluta: Global yields and extract chemical composition, Electron. J. Biotechnol., 10, 141-148 (2007).

[117] B. G. Liu, Z. X. Ning, Y. Zhan, K. Y. Xu, J. H. Gao, Chracterization and 1,1-diphenyl-2-picrylhydrazyl radical scavenging activity of methanol and supercritical carbon dioxide extracts from leaves of Adinandra nitida, J. Food Biochem., 32, 431-442 (2008).

[118] M. Bimakr, R. A. Rahman, A. Ganjloo, F. S. Taip, L. M. Salleh, M. Z. I. Sarker, Optimization of supercritical carbon dioxide extraction of bioactive flavonoid compounds from spearmint (Mentha spicata L.) leaves by using response surface methodology, Food Bioprocess Tech., 5, 912-920 (2012).

[119] M. Bimakr, R. L. A. Rahman, F. S. Taip, A. Ganjloo, L. M. Salleh, J. Selamat, A. Hamid, I. S. M. Zaidul, Comparison of different extraction methods for the extraction of major bioactive flavonoid compounds from spearmint (Mentha spicata 1.) leaves, Food Bioprod. Process., 89, 67-72 (2011).

[120] L. C. Paviani, C. Dariva, M. C. Marcucci, F. A. Cabral, Supercritical carbon dioxide selectivity to fractionate phenolic compounds from the dry ethanolic extract of propolis, J. Food Process Eng., 33, 15-27 (2010).

[121] C. R. Chen, Y. N. Lee, M. R. Lee, C. M. J. Chang, Supercritical fluids extraction of cinnamic acid derivatives from Brazilian propolis and the effect on growth inhibition of colon cancer cells, Journal of the Taiwan Institute of Chemical Engineers, 40, 130135 (2009).

[122] Y. N. Lee, C. R. Chen, H. L. Yang, C. C. Lin, C. M. J. Chang, Isolation and purification of 3,5-diprenyl4-hydroxycinnamic acid (artepillin C) in Brazilian propolis by supercritical fluid extractions, Sep. Purif. Technol., 54, 130-138 (2007).
[123] D. Biscaia, S. R. S. Ferreira, Propolis extracts obtained by low pressure methods and supercritical fluid extraction, J. Supercrit. Fluids, 51, 17-23 (2009).

[124] M. C. Lin, M. J. Tsai, K. C. Wen, Supercritical fluid extraction of flavonoids from Scutellariae radix, $J$. Chromatogr. A, 830, 387-395 (1999).

[125] T. M. Takeuchi, M. L. Rubano, M. A. A. Meireles, Characterization and functional properties of macela (Achyrocline satureioides) extracts obtained by supercritical fluid extraction using mixtures of $\mathrm{CO}_{2}$ plus ethanol, Food Bioprocess Tech., 3, 804-812 (2010).

[126] M. Ligor, O. Kornysova, A. Maruska, B. Buszewski, Determination of flavonoids in tea and rooibos extracts by TLC and HPLC, Jpc-J. Planar. Chromat., 21, 355-360 (2008).

[127] D. Meterc, M. Petermann, E. Weidner, Drying of aqueous green tea extracts using a supercritical fluid spray process, J. Supercrit. Fluids, 45, 253-259 (2008).

[128] J. Shi, H. Nawaz, J. Pohorly, G. Mittal, Y. Kakuda, Y. M. Jiang, Extraction of polyphenolics from plant material for functional foods - engineering and technology, Food Rev. Int., 21, 139-166 (2005).

[129] E. E. Yilmaz, E. B. Ozvural, H. Vural, Extraction and identification of proanthocyanidins from grape seed (Vitis vinifera) using supercritical carbon dioxide, $J$. Supercrit. Fluids, 55, 924-928 (2011).

[130] L. F. Zhang, D. H. Mou, Y. S. Du, Procyanidins: Extraction and micro-encapsulation, J. Sci. Food Agric., 87, 2192-2197 (2007).

[131] T. I. Lafka, A. E. Lazou, V. J. Sinanoglou, E. S. Lazos, Phenolic and antioxidant potential of olive oil mill wastes, Food Chem., 125, 92-98 (2011).

[132] M. E. M. Braga, R. M. S. Santos, I. J. Seabra, R. Facanali, M. O. M. Marques, H. C. de Sousa, Fractioned SFE of antioxidants from maritime pine bark, J. Supercrit. Fluids, 47, 37-48 (2008).

[133] O. Yesil-Celiktas, F. Otto, H. Parlar, A comparative study of flavonoid contents and antioxidant activities of supercritical $\mathrm{CO}_{2}$ extracted pine barks grown in different regions of Turkey and Germany, Eur. Food Res. Technol., 229, 671-677 (2009).

[134] A. T. Serra, I. J. Seabra, M. E. M. Braga, M. R. Bronze, H. C. de Sousa, C. M. M. Duarte, Processing cherries (Prunus avium) using supercritical fluid technology. Part 1: Recovery of extract fractions rich in bioactive compounds, J. Supercrit. Fluids, 55, 184-191 (2010).

[135] F. J. Senorans, E. Ibanez, S. Cavero, J. Tabera, G. Reglero, Liquid chromatographic-mass spectromet- 
ric analysis of supercritical-fluid extracts of rosemary plants, J. Chromatogr. A, 870, 491-499 (2000).

[136] P. Manpong, S. Douglas, P. L. Douglas, S. Pongamphai, W. Teppaitoon, O. Kaewprakaisangkul, Response surface methodology applied to the extraction of phenolic compounds from Jatropha curcas Linn. leaves using supercritical $\mathrm{CO}_{2}$ with a methanol cosolvent, J. Food Process Eng., 34, 1661-1681 (2011).

[137] A. Manosroi, W. Ruksiriwanich, M. Abe, H. Sakai, W. Manosroi, J. Manosroi, Biological activities of the rice bran extract and physical characteristics of its entrapment in niosomes by supercritical carbon dioxide fluid, J. Supercrit. Fluids, 54, 137-144 (2010).

[138] P. Mitra, P. C. Barman, K. S. Chang, Coumarin extraction from Cuscuta reflexa using supercritical fluid carbon dioxide and development of an artificial neural network model to predict the coumarin yield, Food Bioprocess Tech., 4, 737-744 (2011).

[139] C. C. Yang, M. R. Lee, S. L. Hsu, C. M. J. Chang, Supercritical fluids extraction of capillarisin from $\mathrm{Ar}$ temisia capillaris and its inhibition of in vitro growth of hepatoma cells, J. Supercrit. Fluids, 42, 96-103 (2007).

[140] R. F. Rodrigues, A. K. Tashima, R. M. S. Pereira, R. S. Mohamed, F. A. Cabral, Coumarin solubility and extraction from emburana (Torresea cearensis) seeds with supercritical carbon dioxide, J. Supercrit. Flu$i d s, \mathbf{4 3}$, 375-382 (2008).

[141] D. Grigonis, P. R. Venskutonis, B. Sivik, M. Sandahl, C. S. Eskilsson, Comparison of different extraction techniques for isolation of antioxidants from sweet grass (Hierochloe odorata), J. Supercrit. Fluids, 33, 223-233 (2005).

[142] Q. H. Chen, P. Li, J. He, Z. Zhang, J. Liu, Supercritical fluid extraction for identification and determination of volatile metabolites from Angelica dahurica by GC-MS, J. Sep. Sci., 31, 3218-3224 (2008).

[143] Y. Chen, Y. C. Jin, Y. F. Chen, Y. Q. Jin, X. S. Liu, L. H. Wang, A novel HPLC method to analyze imperatorin and isoimperatorin of Angelica dahurica oils obtained by supercritical fluid extraction, J. Liq. Chromatogr. Rel. Technol., 32, 2384-2395 (2009).

[144] A. T. Quitain, K. Oro, S. Katoh, T. Moriyoshi, Recovery of oil components of okara by ethanol-modified supercritical carbon dioxide extraction, Bioresour. Technol., 97, 1509-1514 (2006).

[145] O. I. Pokrovskiï, A. A. Markoliya, F. D. Lepeshkin, I. V. Kuvykin, O. O. Parenago, S. A. Gonchukov, Extraction of linear furocoumarins from Ammi majus seeds by means of supercritical fluid extraction and supercritical fluid chromatography, Russian Journal of Physical Chemistry B, 3, 1165-1171 (2009).

[146] T. Hatami, R. N. Cavalcanti, T. M. Takeuchi, M. A. A. Meireles, Supercritical fluid extraction of bioactive compounds from macela (Achyrocline satureioides) flowers: Kinetic, experiments and modeling, $J$. Supercrit. Fluids, 65, 71-77 (2012).

[147] T. Floris, G. Filippino, S. Scrugli, M. B. Pinna, F. Argiolas, A. Argiolas, M. Murru, E. Reverchon, Antioxidant compounds recovery from grape residues by a supercritical antisolvent assisted process, J. Supercrit. Fluids, 54, 165-170 (2010).

[148] K. Ghafoor, J. Park, Y. H. Choi, Optimization of supercritical fluid extraction of bioactive compounds from grape (Vitis labrusca B.) peel by using response surface methodology, Innov. Food Sci. Emerg., 11, 485-490 (2010).

[149] X. L. Liu, M. M. Zhao, J. S. Wang, W. Luo, Antimicrobial and antioxidant activity of emblica extracts obtained by supercritical carbon dioxide extraction and methanol extraction, J. Food Biochem., 33, 307330 (2009).

[150] M. Sandell, O. Laaksonen, R. Jarvinen, N. Rostiala, T. Pohjanheimo, K. Tiitinen, H. Kallio, Orosensory profiles and chemical composition of black currant (Ribes nigrum) juice and fractions of press residue, $J$. Agric. Food. Chem., 57, 3718-3728 (2009).

[151] İ. H. Adil, M. E. Yener, A. Bayındırl, Extraction of total phenolics of sour cherry pomace by high pressure solvent and subcritical fluid and determination of the antioxidant activities of the extracts, Sep. Sci. Technol., 43, 1091-1110 (2008).

[152] Y. K. Cho, J. H. Ryu, G. B. Lim, S. Y. Lee, Supercritical fluid extraction of glabridin from Glycyrrhiza glabra, Frontiers on Separation Science and Technology, 334-337 (2004).

[153] Q. Chen, S. Yao, X. F. Huang, J. G. Luo, J. S. Wang, L. Y. Kong, Supercritical fluid extraction of Coriandrum sativum and subsequent separation of isocoumarins by high-speed counter-current chromatography, Food Chem., 117, 504-508 (2009).

[154] H. Sovová, L. Opletal, M. Bártlová, M. Sajfrtová, M. Křenková, Supercritical fluid extraction of lignans and cinnamic acid from Schisandra chinensis, $J$. Supercrit. Fluids, 42, 88-95 (2007).

[155] M. C. Wang, Y. C. Lai, C. L. Chang, High throughput screening and antioxidant assay of dibenzo a,c cyclooctadiene lignans in modified-ultrasonic and supercritical fluid extracts of Schisandra chinensis baill by liquid chromatography-mass spectrometry and a free radical-scavenging method, J. Sep. Sci., 31, 1322-1332 (2008). 
[156] E. Sedlak, I. Boldizsar, L. Borsodi, Z. Fuzfai, I. Molnar-Perl, E. Preininger, I. Gyurjan, Identification and quantification of lignans, carboxylic acids and sugars in the leaves of Forsythia species and cultivars, Chromatographia, 68, S35-S41 (2008).

[157] L. M. Comin, F. Temelli, M. A. Saldana, Supercritical $\mathrm{CO}_{2}$ extraction of flax lignans, J. Am. Oil Chem. Soc., 88, 707-715 (2011).

[158] V. A. D. Garcia, V. F. Cabral, E. F. Zanoelo, C. da Silva, L. Cardozo, Extraction of mucuna seed oil using supercritical carbon dioxide to increase the concentration of L-DOPA in the defatted meal, J. Supercrit. Fluids, 69, 75-81 (2012).

[159] O. Laaksonen, M. Sandell, R. Jarvinen, H. Kallio, Orosensory contributing compounds in crowberry (Empetrum nigrum) press-byproducts, Food Chem., 124, 1514-1524 (2011).

[160] K. Hartonen, J. Parshintsev, K. Sandberg, E. Bergelin, L. Nisula, M. L. Riekkola, Isolation of flavonoids from aspen knotwood by pressurized hot water extraction and comparison with other extraction techniques, Talanta, 74, 32-38 (2007).

[161] A. N. Giannuzzo, H. J. Boggetti, M. A. Nazareno, H. T. Mishima, Supercritical fluid extraction of naringin from the peel of Citrus paradisi, Phytochem. Anal, 14, 221-223 (2003).

[162] Y. G. Zu, X. L. Liu, Y. J. Fu, N. Wu, Y. Kong, M. Wink, Chemical composition of the SFE- $\mathrm{CO}_{2}$ extracts from Cajanus cajan (L.) huth and their antimicrobial activity in vitro and in vivo, Phytomedicine, 17, 1095-1101 (2010).

[163] J. Y. Peng, G. R. Fan, Y. F. Chai, Y. T. Wu, Efficient new method for extraction and isolation of three flavonoids from Patrinia villosa Juss. by supercritical fluid extraction and high-speed counter-current chromatography, J. Chromatogr. A, 1102, 44-50 (2006).

[164] Q. H. Chen, P. Li, F. J. Yuan, F. J. Cheng, J. He, J. Liu, Z. Zhang, Identification and quantification of the volatile constituents in Cnidium monnieri using supercritical fluid extraction followed by GC-MS, $J$. Sep. Sci., 32, 252-257 (2009).

[165] L. A. V. Sarmento, R. A. F. Machado, J. C. C. Petrus, T. R. Tamanini, A. Bolzan, Extraction of polyphenols from cocoa seeds and concentration through polymeric membranes, J. Supercrit. Fluids, 45, 64-69 (2008).

[166] V. Lanzotti, The analysis of onion and garlic, J. Chromatogr. A, 1112, 3-22 (2006).

[167] C. Turner, P. Turner, G. Jacobson, K. Almgren, M. Waldeback, P. Sjoberg, E. N. Karlsson, K. E.
Markides, Subcritical water extraction and betaglucosidase-catalyzed hydrolysis of quercetin glycosides in onion waste, Green Chem., 8, 949-959 (2006).

[168] W. L. Yu, B. Shu, Y. P. Zhao, Supercritical $\mathrm{CO}_{2}$ extraction of resveratrol and its glycoside piceid from chinese traditional medicinal herb Polygonum cuspidatum, J. Sci. Food Agric., 85, 489-492 (2005).

[169] V. Jerkovic, S. Collin, Fate of resveratrol and piceid through different hop processings and storage times, J. Agric. Food. Chem., 56, 584-590 (2008).

[170] A. L. Dawidowicz, D. Wianowska, J. Gawdzik, D. H. Smolarz, Optimization of ase conditions for the HPLC determination of rutin and isoquercitrin in sambucus nigra L., J. Liq. Chromatogr. Rel. Technol., 26, 2381-2397 (2003).

[171] H. Y. Li, J. Wu, C. B. Rempel, U. Thiyam, Effect of operating parameters on oil and phenolic extraction using supercritical $\mathrm{CO}_{2}, \mathrm{~J}$. Am. Oil Chem. Soc., 87, 1081-1089 (2010).

[172] M. Sun, L. Xu, M. D. A. Saldana, F. Temelli, Comparison of canola meals obtained with conventional methods and supercritical $\mathrm{CO}_{2}$ with and without ethanol, J. Am. Oil Chem. Soc., 85, 667-675 (2008).

[173] M. Markom, M. Hasan, W. R. W. Daud, N. Anuar, O. Hassan, H. Singh, Chemical profiling and quantification of tannins in Phyllanthus niruri Linn. fractionated by SFE method, Sep. Sci. Technol., 46, 71-78 (2011).

[174] M. Markom, M. Hasan, W. R. W. Daud, H. Singh, J. M. Jahim, Extraction of hydrolysable tannins from Phyllanthus niruri Linn. : Effects of solvents and extraction methods, Sep. Purif. Technol., 52, 487-496 (2007).

[175] Z. Solati, B. S. Baharin, H. Bagheri, Supercritical carbon dioxide $\left(\mathrm{SC}-\mathrm{CO}_{2}\right)$ extraction of Nigella sativa L. oil using full factorial design, Ind. Crop. Prod., 36, 519-523 (2012).

[176] A. S. Zarena, B. Manohar, K. U. Sankar, Optimization of supercritical carbon dioxide extraction of xanthones from mangosteen pericarp by response surface methodology, Food Bioprocess Tech., 5, 1181-1188 (2012).

[177] A. S. Zarena, N. M. Sachindra, K. U. Sankar, Optimisation of ethanol modified supercritical carbon dioxide on the extract yield and antioxidant activity from Garcinia mangostana L., Food Chem., 130, 203-208 (2012).

[178] A. S. Zarena, K. U. Sankar, Supercritical carbon dioxide extraction of xanthones with antioxidant activ- 
ity from Garcinia mangostana: Characterization by HPLC/LC-ESI-MS, J. Supercrit. Fluids, 49, 330 337 (2009).

[179] C. R. Chen, C. H. Wang, L. Y. Wang, Z. H. Hong, S. H. Chen, W. J. Ho, C. M. J. Chang, Supercritical carbon dioxide extraction and deacidification of rice bran oil, J. Supercrit. Fluids, 45, 322-331 (2008).

[180] S. P. Jesus, R. Grimaldi, H. Hense, Recovery of gamma-oryzanol from rice bran oil byproduct using supercritical fluid extraction, J. Supercrit. Fluids, 55, 149-155 (2010).

[181] M. J. Lerma-Garcia, J. M. Herrero-Martinez, E. F. Simo-Alfonso, C. R. B. Mendonca, G. Ramis-Ramos, Composition, industrial processing and applications of rice bran gamma-oryzanol, Food Chem., 115, 389-404 (2009).

[182] B. Shan, J. H. Xie, J. H. Zhu, Y. Peng, Ethanol modified supercritical carbon dioxide extraction of flavonoids from Momordica charantia L. and its antioxidant activity, Food Bioprod. Process., 90, 579-587 (2012).

[183] J. Z. He, P. Shao, J. H. Liu, Q. M. Ru, Supercritical carbon dioxide extraction of flavonoids from pomelo (Citrus grandis (L.) Osbeck) peel and their antioxidant activity, Int. J. Mol. Sci., 13, 13065-13078 (2012).

[184] E. Ibanez, A. Kubatova, F. J. Senorans, S. Cavero, G. Reglero, S. B. Hawthorne, Subcritical water extraction of antioxidant compounds from rosemary plants, J. Agric. Food. Chem., 51, 375-382 (2003).

[185] M. Ollanketo, A. Peltoketo, K. Hartonen, R. Hiltunen, M. L. Riekkola, Extraction of sage (Salvia officinalis L.) by pressurized hot water and conventional methods: Antioxidant activity of the extracts, Eur. Food Res. Technol., 215, 158-163 (2002).

[186] F. J. Senorans, A. Ruiz-Rodriguez, S. Cavero, A. Cifuentes, E. Ibanez, G. Reglero, Isolation of antioxidant compounds from orange juice by using countercurrent supercritical fluid extraction (CC-SFE), $J$. Agric. Food. Chem., 49, 6039-6044 (2001).

[187] C. Simo, E. Ibanez, F. J. Senorans, C. Barbas, G. Reglero, A. Cifuentes, Analysis of antioxidants from orange juice obtained by countercurrent supercritical fluid extraction, using micellar electrokinetic chromatography and reverse-phase liquid chromatography, J. Agric. Food. Chem., 50, 6648-6652 (2002).

[188] A. Petsalo, J. Jalonen, A. Tolonen, Identification of flavonoids of Rhodiola rosea by liquid chromatography-tandem mass spectrometry, J. Chromatogr. $A$, 1112, 224-231 (2006).

[189] M. Ligor, B. Buszewski, Thin layer chromatographic techniques (TLC, OP TLC) for determination of biological activated compounds from herb extracts, J. Liq. Chromatogr. Rel. Technol., 30, 2617-2628 (2007).

[190] E. H. Liu, L. W. Qi, J. Cao, P. Li, C. Y. Li, Y. B. Peng, Advances of modern chromatographic and electrophoretic methods in separation and analysis of flavonoids, Molecules, 13, 2521-2544 (2008).

[191] M. Kivilompolo, V. Oburka, T. Hyotylainen, Comparison of $\mathrm{GC}-\mathrm{MS}$ and $\mathrm{LC}-\mathrm{MS}$ methods for the analysis of antioxidant phenolic acids in herbs, Anal. Bioanal. Chem., 388, 881-887 (2007).

[192] C. D. Stalikas, Extraction, separation, and detection methods for phenolic acids and flavonoids, J. Sep. Sci., 30, 3268-3295 (2007).

[193] B. Klejdus, J. Kopecký, L. Benešová, J. Vacek, Solidphase/supercritical-fluid extraction for liquid chromatography of phenolic compounds in freshwater microalgae and selected cyanobacterial species, $J$. Chromatogr. A, 1216, 763-771 (2009).

[194] A. Crego, E. Ibanez, E. Garcia, R. R. de Pablos, F. J. Senorans, G. Reglero, A. Cifuentes, Capillary electrophoresis separation of rosemary antioxidants from subcritical water extracts, Eur. Food Res. Technol., 219, 549-555 (2004).

[195] S. F. Miao, J. P. Yu, Z. Du, Y. X. Guan, S. J. Yao, Z. Q. $\mathrm{Zhu}$, Supercritical fluid extraction and micronization of ginkgo flavonoids from Ginkgo biloba leaves, Ind. Eng. Chem. Res., 49, 5461-5466 (2010).

[196] X. Xu, L. P. Sun, J. Dong, H. C. Zhang, Breaking the cells of rape bee pollen and consecutive extraction of functional oil with supercritical carbon dioxide, Innov. Food Sci. Emerg., 10, 42-46 (2009).

[197] P. Ramirez, M. R. Garcia-Risco, S. Santoyo, F. J. Senorans, E. Ibanez, G. Reglero, Isolation of functional ingredients from rosemary by preparative-supercritical fluid chromatography (PREP-SFC), J. Pharm. Biomed. Anal., 41, 1606-1613 (2006).

[198] P. Ramirez, T. Fornari, F. J. Senorans, E. Ibanez, G. Reglero, Isolation of phenolic antioxidant compounds by SFC, J. Supercrit. Fluids, 35, 128-132 (2005).

[199] A. V. M. Nunes, A. A. Matias, M. N. da Ponte, C. M. M. Duarte, Quaternary phase equilibria for $\mathrm{SCCO}_{2}$ plus biophenolic compound plus water plus ethanol, J. Chem. Eng. Data, 52, 244-247 (2007).

[200] J. Chumpoo, P. Prasassarakich, Bio-oil from hydroliquefaction of bagasse in supercritical ethanol, Energy \& Fuels, 24, 2071-2077 (2010).

[201] F. Shahidi, M. Naczk, Food Phenolics: Sources, Chemistry, Effects, Applications, Technomic Publishing Company, 1995. 
[202] S. K. T. Venkatachallam, H. Pattekhan, S. Divakar, U. S. Kadimi, Chemical composition of Nigella sativa L. seed extracts obtained by supercritical carbon dioxide, J. Food Sci. Tech. MYS, 47, 598-605 (2010).

[203] A. Carrasco-Pancorbo, L. Cerretani, A. Bendini, A. Segura-Carretero, T. Gallina-Toschi, A. FernandezGutierrez, Analytical determination of polyphenols in olive oils, J. Sep. Sci., 28, 837-858 (2005).

[204] L. H. Wang, Y. H. Mei, F. Wang, X. S. Liu, Y. Chen, A novel and efficient method combining SFE and liquid-liquid extraction for separation of coumarins from Angelica dahurica, Sep. Purif. Technol., 77, 397-401 (2011)

[205] P. Rubiolo, B. Sgorbini, E. Liberto, C. Cordero, C. Bicchi, Essential oils and volatiles: Sample preparation and analysis. A review, Flavour Fragrance J., 25, 282-290 (2010).

[206] H. Abbasi, K. Rezaei, L. Rashidi, Extraction of essential oils from the seeds of pomegranate using organic solvents and supercritical $\mathrm{CO}_{2}, J$. Am. Oil Chem. Soc., 85, 83-89 (2008).

[207] B. Damjanović, Z. Lepojević, V. Živković, A. Tolić, Extraction of fennel (Foeniculum vulgare Mill.) seeds with supercritical $\mathrm{CO}_{2}$ : Comparison with hydrodistillation, Food Chem., 92, 143-149 (2005).

[208] J. M. del Valle, T. Rogalinski, C. Zetzl, G. Brunner, Extraction of boldo (Peumus boldus M.) leaves with supercritical $\mathrm{CO}_{2}$ and hot pressurized water, Food Res. Int., 38, 203-213 (2005).

[209] E. S. Giray, S. Kirici, D. A. Kaya, M. Turk, O. Sonmez, M. Inan, Comparing the effect of sub-critical water extraction with conventional extraction methods on the chemical composition of Lavandula stoechas, Talanta, 74, 930-935 (2008).

[210] A. Z. Kakasy, E. Lemberkovics, B. Simandi, L. Lelik, E. Hethelyi, I. Antal, E. Szoke, Comparative study of traditional essential oil and supercritical fluid extracts of Moldavian dragonhead (Dracocephalum moldavica L.), Flavour Fragrance J., 21, 598-603 (2006).

[211] P. Kotnik, M. Škerget, Ž. Knez, Supercritical fluid extraction of chamomile flower heads: Comparison with conventional extraction, kinetics and scale-up, J. Supercrit. Fluids, 43, 192-198 (2007).

[212] M. Lo Presti, S. Ragusa, A. Trozzi, P. Dugo, F. Visinoni, A. Fazio, G. Dugo, L. Mondello, A comparison between different techniques for the isolation of rosemary essential oil, J. Sep. Sci., 28, 273-280 (2005).

[213] S. Shen, Y. F. Sha, C. H. Deng, D. X. Fu, J. K. Chen, X. M. Zhang, Comparison of solid-phase microextraction, supercritical fluid extraction, steam distillation, and solvent extraction techniques for analysis of volatile consituents in fructus amomi, J. AOAC Int., 88, 418-423 (2005).

[214] E. Stashenko, C. Ruiz, A. Munoz, M. Castaneda, J. Martinez, Composition and antioxidant activity of essential oils of Lippia origanoides HBK grown in Colombia, Nat. Prod. Commun., 3, 563-566 (2008).

[215] L. H. Wang, Y. S. Chen, Y. T. Song, Y. Chen, X. S. Liu, GC-MS of volatile components of Schisandra chinensis obtained by supercritical fluid and conventional extraction, J. Sep. Sci., 31, 3238-3245 (2008).

[216] L. H. C. Carlson, A. Bolzan, R. A. F. Machado, Separation of d-limonene from supercritical $\mathrm{CO}_{2}$ by means of membranes, J. Supercrit. Fluids, 34, 143147 (2005).

[217] G. Flores, G. P. Blanch, M. L. R. del Castillo, M. Herraiz, Enantiomeric composition studies in Lavandula species using supercritical fluids, J. Sep. Sci., 28, 2333-2338 (2005).

[218] U. Akman, N. Okay, O. Hortacsu, Hierarchical clustering analysis for the distribution of origanum-oil components in dense $\mathrm{CO}_{2}$, Korean J. Chem. Eng., 25, 329-344 (2008).

[219] F. Gironi, M. Maschietti, Continuous countercurrent deterpenation of lemon essential oil by means of supercritical carbon dioxide: Experimental data and process modelling, Chem. Eng. Sci., 63, 651-661 (2008).

[220] S. Varona, A. Martin, M. J. Cocero, T. Gamse, Supercritical carbon dioxide fractionation of lavandin essential oil: Experiments and modeling, J. Supercrit. Fluids, 45, 181-188 (2008).

[221] S. Raeissi, S. Diaz, S. Espinosa, C. J. Peters, E. A. Brignole, Ethane as an alternative solvent for supercritical extraction of orange peel oils, J. Supercrit. Fluids, 45, 306-313 (2008).

[222] F. Ashtiani, F. Sefidkon, Y. Yamini, K. Khajeh, Supercritical carbon dioxide extraction of volatile components from two eucalyptus species (E. spathulata and E. microtheca), J. Ess. Oil Bear. Pl., 10, 198-208 (2007).

[223] N. Ganan, E. A. Brignole, Fractionation of essential oils with biocidal activity using supercritical $\mathrm{CO}_{2}$ Experiments and modeling, J. Supercrit. Fluids, 58, 58-67 (2011).

[224] S. B. Glišić, M. Ristić, D. U. Skala, The combined extraction of sage (Salvia officinalis L.): Ultrasound followed by supercritical $\mathrm{CO}_{2}$ extraction, Ultrason. Sonochem., 18, 318-326 (2011).

[225] J. Ivanović, S. Đilas, M. Jadranin, V. Vajs, N. Babović, S. Petrović, I. Zizović, Supercritical carbon 
dioxide extraction of antioxidants from rosemary (Rosmarinus officinalis L.) and sage (Salvia officinalis L.), J. Serb. Chem. Soc., 74, 717-732 (2009).

[226] E. Langa, G. Della Porta, A. M. F. Palavra, J. S. Urieta, A. M. Mainar, Supercritical fluid extraction of Spanish sage essential oil: Optimization of the process parameters and modelling, J. Supercrit. Fluids, 49, 174-181 (2009).

[227] C. Grosso, A. C. Figueiredo, J. Burillo, A. M. Mainar, J. S. Urieta, J. G. Barroso, J. A. Coelho, A. M. F. Palavra, Supercritical fluid extraction of the volatile oil from Santolina chamaecyparissus, J. Sep. Sci., 32, 3215-3222 (2009).

[228] J. Ivanović, D. Mišić, M. Ristić, O. Pešić, I. Zizović, Supercritical $\mathrm{CO}_{2}$ extract and essential oil of bay (Laurus nobilis L.) chemical composition and antibacterial activity, J. Serb. Chem. Soc., 75, 395-404 (2010).

[229] H. Marzouki, A. Piras, B. Marongiu, A. Rosa, M. A. Dessi, Extraction and separation of volatile and fixed oils from berries of Laurus nobilis L. by supercritical $\mathrm{CO}_{2}$, Molecules, 13, 1702-1711 (2008).

[230] Z. T. Jiang, R. Li, Y. Wang, Essential oil composition of Cinnamomum loureiroi grown in China extracted by supercritical fluid extraction, J. Ess. Oil Bear. Pl., 11, 267-270 (2008)

[231] P. F. Leal, F. C. M. Chaves, L. C. Ming, A. J. Petenate, M. A. A. Meireles, Global yields, chemical compositions and antioxidant activities of clove basil (Ocimum gratissimum L.) extracts obtained by supercritical fluid extraction, J. Food Process Eng., 29, 547-559 (2006).

[232] T. S. Mann, G. D. K. Babu, S. Guleria, B. Singh, Comparison of Eucalyptus cinerea essential oils produced by hydrodistillation and supercritical carbon dioxide extraction, Nat. Prod. Commun., 6, 107-110 (2011).

[233] M. Sokolova, A. Orav, M. Koel, T. Kailas, M. Muurisepp, Composition of the oil and supercritical fluid $\mathrm{CO}_{2}$ extract of sweet gale (Myrica gale L.) fruits, J. Essent. Oil Res., 17, 188-191 (2005).

[234] I. Zizović, M. Stamenić, A. Orlović, D. Skala, Supercritical carbon dioxide essential oil extraction of lamiaceae family species: Mathematical modelling on the micro-scale and process optimization, Chem. Eng. Sci., 60, 6747-6756 (2005).

[235] R. Saini, V. Jaitak, S. Guleria, V. K. Kaul, G. D. K. Babu, B. Singh, B. Lal, R. D. Singh, Comparison of headspace analysis of volatile constituents with GCMS analysis of hydrodistilled and supercritical fluid extracted oil of Capillipedium parviflorum, J. Essent. Oil Res., 24, 315-320 (2012).
[236] T. C. Tzeng, Y. L. Lin, T. T. Jong, C. M. J. Chang, Ethanol modified supercritical fluids extraction of scopoletin and artemisinin from Artemisia annua L., Sep. Purif. Technol., 56, 18-24 (2007).

[237] M. S. T. Barroso, G. Villanueva, A. M. Lucas, G. P. Perez, R. M. F. Vargas, G. W. Brun, E. Cassel, Supercritical fluid extraction of volatile and non-volatile compounds from Schinus molle L., Braz. J. Chem. Eng., 28, 305-312 (2011).

[238] L. Petrović, Z. Lepojević, V. Sovilj, D. Adamović, V. Tešević, Composition of essential oil obtained from tubular, head and ligulate flowers of Calendula officinalis L. by steam distillation of plant material and $\mathrm{CO}_{2}$ extracts, J. Essent. Oil Res., 22, 143-146 (2010).

[239] P. Costa, S. Goncalves, C. Grosso, P. B. Andrade, P. Valentao, M. G. Bernardo-Gil, A. Romano, Chemical profiling and biological screening of Thymus lotocephalus extracts obtained by supercritical fluid extraction and hydrodistillation, Ind. Crop. Prod., 36, 246-256 (2012).

[240] N. F. Ramandi, N. M. Najafi, F. Raofie, E. Ghasemi, Central composite design for the optimization of supercritical carbon dioxide fluid extraction of fatty acids from Borago officinalis L. flower, J. Food Sci., 76, C1262-C1266 (2011).

[241] L. T. Danh, D. A. T. Ngo, T. N. H. Le, J. Zhao, R. Mammucari, N. Foster, Antioxidant activity, yield and chemical composition of lavender essential oil extracted by supercritical $\mathrm{CO}_{2}$, J. Supercrit. Fluids, 70, 27-34 (2012).

[242] S. M. Ghoreishi, H. Kamali, H. S. Ghaziaskar, A. A. Dadkhah, Optimization of supercritical extraction of linalyl acetate from lavender via box-behnken design, Chem. Eng. Technol., 35, 1641-1648 (2012).

[243] P. Costa, C. Grosso, S. Goncalves, P. B. Andrade, P. Valentao, M. G. Bernardo-Gil, A. Romano, Supercritical fluid extraction and hydrodistillation for the recovery of bioactive compounds from Lavandula viridis L'Her, Food Chem., 135, 112-121 (2012).

[244] O. Bensebia, D. Barth, B. Bensebia, A. Dahmani, Supercritical $\mathrm{CO}_{2}$ extraction of rosemary: Effect of extraction parameters and modelling, J. Supercrit. Fluids, 49, 161-166 (2009).

[245] S. Cavero, L. Jaime, P. J. Martin-Alvarez, F. J. Senorans, G. Reglero, E. Ibanez, In vitro antioxidant analysis of supercritical fluid extracts from rosemary (Rosmarinus officinalis L.), Eur. Food Res. Technol., 221, 478-486 (2005).

[246] M. Herrero, M. Plaza, A. Cifuentes, E. Ibanez, Green processes for the extraction of bioactives from rosemary: Chemical and functional characterization via ultra-performance liquid chromatography-tandem 
mass spectrometry and in-vitro assays, J. Chromatogr. A, 1217, 2512-2520 (2010).

[247] S. Irmak, K. Solakyildirim, A. Hesenov, O. Erbatur, Study on the stability of supercritical fluid extracted rosemary (Rosmarinus offcinalis L.) essential oil, $J$. Anal. Chem., 65, 899-906 (2010).

[248] C. F. Kuo, J. D. Su, C. H. Chiu, C. C. Peng, C. H. Chang, T. Y. Sung, S. H. Huang, W. C. Lee, C. C. Chyau, Anti-inflammatory effects of supercritical carbon dioxide extract and its isolated carnosic acid from Rosmarinus officinalis leaves, J. Agric. Food. Chem., 59, 3674-3685 (2011).

[249] K. A. McCormick, J. S. Olivarez, R. A. Fisher, T. M. Nahir, C. L. Phelps, Effect of sample preparation on the amounts of alpha-pinene and verbenone extracted from rosemary, J. Essent. Oil Res., 18, 478-480 (2006).

[250] S. A. Rezzoug, C. Boutekedjiret, K. Allaf, Optimization of operating conditions of rosemary essential oil extraction by a fast controlled pressure drop process using response surface methodology, J. Food Eng., 71, 9-17 (2005).

[251] C. H. Peng, J. D. Su, C. C. Chyau, T. Y. Sung, S. S. Ho, C. C. Peng, R. Y. Peng, Supercritical fluid extracts of rosemary leaves exhibit potent anti-inflammation and anti-tumor effects, Biosci., Biotechnol., Biochem., 71, 2223-2232 (2007).

[252] A. Visentin, M. Cismondi, D. Maestri, Supercritical $\mathrm{CO}_{2}$ fractionation of rosemary ethanolic oleoresins as a method to improve carnosic acid recovery, Innov. Food Sci. Emerg., 12, 142-145 (2011).

[253] O. Yesil-Celiktas, E. Bedir, F. V. Sukan, In vitro antioxidant activities of Rosmarinus officinalis extracts treated with supercritical carbon dioxide, Food Chem., 101, 1457-1464 (2007).

[254] C. Soler-Rivas, F. R. Marin, S. Santoyo, M. R. Garcia-Risco, F. J. Senorans, G. Reglero, Testing and enhancing the in vitro bioaccessibility and bioavailability of Rosmarinus officinalis extracts with a high level of antioxidant abietanes, J. Agric. Food. Chem., 58, 1144-1152 (2010).

[255] J. Ivanović, I. Zizović, M. Ristić, M. Stamenić, D. Skala, The analysis of simultaneous clove/oregano and clove/thyme supercritical extraction, J. Supercrit. Fluids, 55, 983-991 (2011).

[256] A. Ocana-Fuentes, E. Arranz-Gutierrez, F. J. Senorans, G. Reglero, Supercritical fluid extraction of oregano (Origanum vulgare) essentials oils: Antiinflammatory properties based on cytokine response on THP-1 macrophages, Food Chem. Toxicol., 48, 1568-1575 (2010).
[257] M. Khajeh, Optimization of process variables for essential oil components from Satureja hortensis by supercritical fluid extraction using box-behnken experimental design, J. Supercrit. Fluids, 55, 944-948 (2011).

[258] C. Grosso, A. C. Figueiredo, J. Burillo, A. M. Mainar, J. S. Urieta, J. G. Barroso, J. A. Coelho, A. M. F. Palavra, Enrichment of the thymoquinone content in volatile oil from Satureja montana using supercritical fluid extraction, J. Sep. Sci., 32, 328-334 (2009).

[259] O. P. Nautiyal, K. K. Tiwari, Extraction of dill seed oil (Anethum sowa) using supercritical carbon dioxide and comparison with hydrodistillation, Ind. Eng. Chem. Res., 50, 5723-5726 (2011).

[260] P. P. Almeida, N. Mezzomo, S. R. S. Ferreira, Extraction of Mentha spicata L. volatile compounds: Evaluation of process parameters and extract composition, Food Bioprocess Tech., 5, 548-559 (2012).

[261] J. Ivanović, M. Ristić, D. Skala, Supercritical $\mathrm{CO}_{2}$ extraction of Helichrysum italicum: Influence of $\mathrm{CO}_{2}$ density and moisture content of plant material, J. Supercrit. Fluids, 57, 129-136 (2011).

[262] T. Hatami, M. Rahimi, P. C. Veggi, R. Portillo-Prieto, M. A. A. Meireles, Near-critical carbon dioxide extraction of khoa (Satureja boliviana Benth Briq) using ethanol as a co-solvent: Experiment and modeling, J. Supercrit. Fluids, 55, 929-936 (2011).

[263] D. Missopolinou, C. Tsioptsias, C. Lambrou, C. Panayiotou, Selective extraction of oxygenated compounds from oregano with sub-critical water, J. Sci. Food Agric., 92, 814-820 (2012).

[264] L. Martin, A. Gonzalez-Coloma, C. E. Diaz, A. M. Mainar, J. S. Urieta, Supercritical $\mathrm{CO}_{2}$ extraction of Persea indica: Effect of extraction parameters, modelling and bioactivity of its extracts, J. Supercrit. Fluids, 57, 120-128 (2011).

[265] P. F. de Oliveira, R. A. F. Machado, A. Bolzan, D. Barth, Supercritical fluid extraction of hernandulcin from Lippia dulcis Trev., J. Supercrit. Fluids, 63, 161-168 (2012).

[266] B. Marongiu, A. Piras, S. Porcedda, E. Tuveri, E. Sanjust, M. Meli, F. Sollai, P. Zucca, A. Rescigno, Supercritical $\mathrm{CO}_{2}$ extract of Cinnamomum zeylanicum: Chemical characterization and antityrosinase activity, J. Agric. Food. Chem., 55, 10022-10027 (2007).

[267] G. Y. Zhang, J. Y. Ling, Z. J. Cui, Supercritical $\mathrm{CO}_{2}$ extraction of essential oil from Dracocephalum tanguticum max-im and analysis by GC-MS, J. Liq. Chromatogr. Rel. Technol., 30, 287-292 (2007). 
[268] L. Martin, A. M. Mainar, A. Gonzalez-Coloma, J. Burillo, J. S. Urieta, Supercritical fluid extraction of wormwood (Artemisia absinthium L.), J. Supercrit. Fluids, 56, 64-71 (2011).

[269] H. Baseri, A. Haghighi-Asl, M. N. Lotfollahi, Effects of operating parameters on the cinnamaldehyde content of extracted essential oil using various methods, Chem. Eng. Technol., 33, 267-274 (2010).

[270] H. Baseri, M. N. Lotfollahi, A. H. Asl, Effects of some experimental parameters on yield and composition of supercritical carbon dioxide extracts of cinnamon bark, J. Food Process Eng., 34, 293-303 (2011).

[271] H. K. P. Ha, J. Maridable, P. Gaspillo, M. Hasika, R. Malaluan, J. Kawasaki, Essential oil from lemongrass extracted by supercritical carbon dioxide and steam distillation, Philipp. Agric. Sci., 91, 36-41 (2008).

[272] R. M. F. Vargas, E. Cassel, G. M. F. Gomes, L. G. S. Longhi, L. Atti-Serafini, A. C. Atti-Santos, Supercritical extraction of carqueja essential oil: Experiments and modeling, Braz. J. Chem. Eng., 23, 375-382 (2006).

[273] A. Ranalli, S. Contento, L. Lucera, G. Pavone, G. Di Giacomo, L. Aloisio, C. Di Gregorio, A. Mucci, I. Kourtikakis, Characterization of carrot root oil arising from supercritical fluid carbon dioxide extraction, J. Agric. Food. Chem., 52, 4795-4801 (2004).

[274] M. Khajeh, Optimisation of supercritical fluid extraction of essential oil components of Diplotaenia cachrydifolia: Box-Behnken design, Nat. Prod. Res., 26, 1926-1930 (2012).

[275] W. Q. Guan, S. F. Li, R. X. Yan, S. K. Tang, C. Quan, Comparison of essential oils of clove buds extracted with supercritical carbon dioxide and other three traditional extraction methods, Food Chem., 101, 1558-1564 (2007).

[276] F. Yazdani, M. Mafi, F. Farhadi, K. Tabar-Heidar, K. Aghapoor, F. Mohsenzadeh, H. R. Darabi, Supercritical $\mathrm{CO}_{2}$ extraction of essential oil from clove bud: Effect of operation conditions on the selective isolation of eugenol and eugenyl acetate, Z. Naturforsh. B, 60 , 1197-1201 (2005).

[277] X. G. Shi, X. Wang, D. J. Wang, Y. L. Geng, J. H. Liu, Separation and purification of cyperone from Cyperus rotundus with supercritical fluid extraction and high-speed counter-current chromatography, Sep. Sci. Technol., 44, 712-721 (2009).

[278] M. Khajeh, Y. Yamini, S. Shariati, Comparison of essential oils compositions of Nepeta persica obtained by supercritical carbon dioxide extraction and steam distillation methods, Food Bioprod. Process., 88, 227-232 (2010).
[279] A. Peterson, S. Machmudah, B. C. Roy, M. Goto, M. Sasaki, T. Hirose, Extraction of essential oil from geranium (Pelargonium graveolens) with supercritical carbon dioxide, J. Chem. Technol. Biotechnol., 81, 167-172 (2006).

[280] C. E. Vargas, M. F. Mendes, D. A. Azevedo, F. L. P. Pessoa, A. C. Uller, Extraction of the essential oil of abajeru (Chrysobalanus icaco) using supercritical $\mathrm{CO}_{2}$, J. Supercrit. Fluids, 54, 171-177 (2010).

[281] B. Marongiu, A. Piras, S. Porcedda, E. Tuveri, Extraction of Santalum album and Boswellia carterii Birdw. volatile oil by supercritical carbon dioxide: Influence of some process parameters, Flavour Fragrance J., 21, 718-724 (2006).

[282] T. D. Luu, P. Truong, R. Mammucari, N. Foster, Extraction of vetiver essential oil by ethanol-modified supercritical carbon dioxide, Chem. Eng. J., 165, 26-34 (2010).

[283] M. Khajeh, M. G. Moghaddam, M. Shakeni, Application of artificial neural network in predicting the extraction yield of essential oils of Diplotaenia cachrydifolia by supercritical fluid extraction, $J$. Supercrit. Fluids, 69, 91-96 (2012).

[284] T. Tapia, F. Perich, F. Pardo, G. Palma, A. Quiroz, Identification of volatiles from differently aged red clover (Trifolium pratense) root extracts and behavioural responses of clover root borer (Hylastinus obscurus) (marsham) (Coleoptera : Scolytidae) to them, Biochem. Syst. Ecol., 35, 61-67 (2007).

[285] Z. Zeković, Z. Lepojević, S. Milić, D. Adamović, I. Mujić, Supercritical $\mathrm{CO}_{2}$ extraction of mentha (Mentha piperita L.) at different solvent densities, J. Serb. Chem. Soc., 74, 417-425 (2009).

[286] B. Damjanović, D. Skala, J. Baras, D. Petrović-Djakov, Isolation of essential oil and supercritical carbon dioxide extract of Juniperus communis L. fruits from Montenegro, Flavour Fragrance J., 21, 875-880 (2006).

[287] M. Izadifar, F. Abdolahi, Comparison between neural network and mathematical modeling of supercritical $\mathrm{CO}_{2}$ extraction of black pepper essential oil, J. Supercrit. Fluids, 38, 37-43 (2006).

[288] C. Perakis, V. Louli, K. Magoulas, Supercritical fluid extraction of black pepper oil, J. Food Eng., 71, 386-393 (2005).

[289] Y. Yamini, N. Bahramifar, F. Sefidkon, M. J. Saharkhiz, E. Salamifar, Extraction of essential oil from Pimpinella anisum using supercritical carbon dioxide and comparison with hydrodistillation, Nat. Prod. Res., 22, 212-218 (2008). 
[290] S. A. Yang, E. J. Lee, H. D. Choi, M. H. Yu, M. H. Bang, I. S. Lee, S. P. Lee, Anti-allergy effect of marc from silver vine (Actinidia polygama) prepared by supercritical fluid extraction, Food Sci. Biotechnol., 19, 1309-1316 (2010).

[291] B. Marongiu, A. Piras, S. Porcedda, E. Tuveri, A. Maxia, Comparative analysis of the oil and supercritical $\mathrm{CO}_{2}$ extract of Ridolfia segetum (L.) Moris, Nat. Prod. Res., 21, 412-417 (2007).

[292] H. Kazazi, K. Rezaei, S. J. Ghotb-Sharif, Z. EmamDjomeh, Y. Yamini, Supercriticial fluid extraction of flavors and fragrances from Hyssopus officinalis L. cultivated in Iran, Food Chem., 105, 805-811 (2007).

[293] B. Marongiu, A. Piras, S. Porcedda, E. Tuveri, A. Maxia, Isolation of Seseli bocconi Guss., subsp praecox gamisans (Apiaceae) volatile oil by supercritical carbon dioxide extraction, Nat. Prod. Res., 20, 820 826 (2006).

[294] Z. N. Yang, S. Q. Luo, Q. C. Peng, C. Zhao, Z. W. $\mathrm{Yu}, \mathrm{GC}-\mathrm{MS}$ analysis of the essential oil of coral ginger (Zingiber corallinum Hance) rrhizome obtained by supercritical fluid extraction and steam distillation extraction, Chromatographia, 69, 785-790 (2009).

[295] I. S. Marković, Z. A. Darmati, B. F. Abramović, Chemical composition of leaf extracts of Stevia rebaudiana Bertoni grown experimentally in Vojvodina, J. Serb. Chem. Soc., 73, 283-297 (2008).

[296] B. K. Huang, L. P. Qin, Q. C. Chu, Q. Y. Zhang, L. H. Gao, H. C. Zheng, Comparison of headspace SPME with hydrodistillation and SFE for analysis of the volatile components of the roots of Valeriana officinalis var. latifolia, Chromatographia, 69, 489-496 (2009).

[297] A. Salimi, S. Fatemi, H. Z. N. Nei, A. Safaralie, Mathematical modeling of supercritical extraction of valerenic acid from Valeriana officinalis L., Chem. Eng. Technol., 31, 1470-1480 (2008).

[298] P. M. Moura, G. H. C. Prado, M. A. A. Meireles, C. G. Pereira, Supercritical fluid extraction from guava (Psidium guajava) leaves: Global yield, composition and kinetic data, J. Supercrit. Fluids, 62, 116-122 (2012).

[299] S. M. Pourmortazavi, M. Ghadiri, S. S. Hajimirsadeghi, Supercritical fluid extraction of volatile components from Bunium persicum Boiss. (black cumin) and Mespilus germanica L. (Medlar) seeds, J. Food Compos. Anal., 18, 439-446 (2005).

[300] F. Sahena, I. S. M. Zaidul, S. Jinap, A. A. Karim, K. A. Abbas, N. A. N. Norulaini, A. K. M. Omar, Application of supercritical $\mathrm{CO}_{2}$ in lipid extraction - A review, J. Food Eng., 95, 240-253 (2009).
[301] R. L. Mendes, A. D. Reis, A. F. Palavra, Supercritical $\mathrm{CO}_{2}$ extraction of gamma-linolenic acid and other lipids from Arthrospira (spirulina) maxima: Comparison with organic solvent extraction, Food Chem., 99, 57-63 (2006).

[302] B. M. Bhosle, R. Subramanian, New approaches in deacidification of edible oils - A review, J. Food Eng., 69, 481-494 (2005).

[303] L. L. Lai, K. C. Soheili, W. E. Artz, Deacidification of soybean oil using membrane processing and subcritical carbon dioxide, J. Am. Oil Chem. Soc., 85, 189-196 (2008).

[304] N. Al-Darmaki, T. Lu, B. Al-Duri, J. B. Harris, T. L. F. Favre, K. Bhaggan, R. C. D. Santos, Isothermal and temperature gradient supercritical fluid extraction and fractionation of squalene from palm fatty acid distillate using compressed carbon dioxide, $J$. Supercrit. Fluids, 61, 108-114 (2012).

[305] F. Meyer, P. Jaeger, R. Eggers, M. Stamenić, S. Milovanović, I. Zizović, Effect of $\mathrm{CO}_{2}$ pre-treatment on $\mathrm{SC}-\mathrm{CO}_{2}$ extraction of natural material, Chem. Eng. Process., 56, 37-45 (2012).

[306] T. Fornari, C. F. Torres, F. J. Senorans, G. Reglero, Simulation and optimization of supercritical fluid purification of phytosterol esters, AlChE J., 55, 10231029 (2009).

[307] M. F. Mendes, F. L. P. Pessoa, G. V. Coelho, A. M. C. Uller, Recovery of the high aggregated compounds present in the deodorizer distillate of the vegetable oils using supercritical fluids, J. Supercrit. Fluids, 34, 157-162 (2005).

[308] M. F. Mendes, F. L. P. Pessoa, A. M. C. Uller, Optimization of the process of concentration of vitamin $\mathrm{E}$ from DDSO using supercritical $\mathrm{CO}_{2}$, Braz. J. Chem. Eng., 22, 83-91 (2005).

[309] C. F. Torres, T. Fornari, G. Torrelo, F. J. Senorans, G. Reglero, Production of phytosterol esters from soybean oil deodorizer distillates, Eur. J. Lipid Sci. Technol., 111, 459-463 (2009).

[310] L. Vazquez, C. F. Torres, T. Fornari, F. J. Senorans, G. Reglero, Recovery of squalene from vegetable oil sources using countercurrent supercritical carbon dioxide extraction, J. Supercrit. Fluids, 40, 59-66 (2007).

[311] R. C. Pradhan, V. Meda, P. K. Rout, S. Naik, A. K. Dalai, Supercritical $\mathrm{CO}_{2}$ extraction of fatty oil from flaxseed and comparison with screw press expression and solvent extraction processes, J. Food Eng., 98, 393-397 (2010).

[312] M. Dong, T. H. Walker, Production and recovery of polyunsaturated fatty acids-added lipids from fer- 
mented canola, Bioresour. Technol., 99, 8504-8506 (2008).

[313] L. Ilic, M. Skerget, M. K. Hrncic, Z. Knez, Phase behavior of sunflower oil and soybean oil in propane and sulphur hexafluoride, J. Supercrit. Fluids, 51, 109-114 (2009).

[314] T. L. Da Silva, E. C. Bernardo, B. Nobre, R. L. Mendes, A. Reis, Extraction of victoria and red globe grape seed oils using supercritical carbon dioxide with and without ethanol, J. Food Lipids, 15, 356369 (2008).

[315] C. S. G. Kitzberger, A. Smania, R. C. Pedrosa, S. R. S. Ferreira, Antioxidant and antimicrobial activities of shiitake (Lentinula edodes) extracts obtained by organic solvents and supercritical fluids, J. Food Eng., 80, 631-638 (2007).

[316] J. Yu, J. Wang, C. M. Liu, Z. Q. Liu, Q. Wang, Application of response surface methodology to optimise supercritical carbon dioxide extraction of oil from rapeseed (Brassica napus L.), Int. J. Food Sci. Technol., 47, 1115-1121 (2012).

[317] F. J. Eller, S. C. Cermak, S. L. Taylor, Supercritical carbon dioxide extraction of cuphea seed oil, Ind. Crop. Prod., 33, 554-557 (2011).

[318] S. C. Liu, F. Yang, C. H. Zhang, H. W. Ji, P. Z. Hong, C. J. Deng, Optimization of process parameters for supercritical carbon dioxide extraction of passiflora seed oil by response surface methodology, J. Supercrit. Fluids, 48, 9-14 (2009).

[319] J. Z. Yin, Q. Q. Xu, W. Wei, A. Q. Wanw, Experiments and numerical simulations of supercritical fluid extraction for Hippophae rhamnoides L. seed oil based on artificial neural networks, Ind. Eng. Chem. Res., 44, 7420-7427 (2005).

[320] Y. Cao, Y. R. Suo, Extraction of microula sikkimensis seed oil and simultaneous analysis of saturated and unsaturated fatty acids by fluorescence detection with reversed-phase HPLC, J. Food Compos. Anal., 23, 100-106 (2010).

[321] H. M. Rui, L. Y. Zhang, Z. W. Li, Y. L. Pan, Extraction and characteristics of seed kernel oil from white pitaya, J. Food Eng., 93, 482-486 (2009).

[322] H. L. N. Lau, Y. M. Choo, A. N. Ma, C. H. Chuah, Characterization and supercritical carbon dioxide extraction of palm oil (Elaeis guineensis), J. Food Lip$i d s$, 13, 210-221 (2006).

[323] E. Arnaiz, J. Bernal, M. T. Martin, C. Garcia-Viguera, J. L. Bernal, L. Toribio, Supercritical fluid extraction of lipids from broccoli leaves, Eur. J. Lipid Sci. Technol., 113, 479-486 (2011).
[324] E. Jenab, K. Rezaei, Z. Emam-Djomeh, Canola oil extracted by supercritical carbon dioxide and a commercial organic solvent (Vol. 108, pg. 488, 2006), Eur. J. Lipid Sci. Technol., 108, 794-794 (2006).

[325] M. M. Pederssetti, F. Palu, E. A. da Silva, J. H. Rohling, L. Cardozo, C. Dariva, Extraction of canola seed (Brassica napus) oil using compressed propane and supercritical carbon dioxide, J. Food Eng., 102, 189-196 (2011).

[326] C. E. Seal, I. Kranner, H. W. Pritchard, Quantification of seed oil from species with varying oil content using supercritical fluid extraction, Phytochem. Anal, 19, 493-498 (2008).

[327] M. Ismail, A. Mariod, G. Bagalkotkar, H. S. Ling, Fatty acid composition and antioxidant activity of oils from two cultivars of cantaloupe extracted by supercritical fluid extraction, Grasas Aceites, 61, 37-44 (2010).

[328] I. M. G. Lopes, M. G. Bernardo-Gil, Characterisation of acorn oils extracted by hexane and by supercritical carbon dioxide, Eur. J. Lipid Sci. Technol., 107, 12-19 (2005).

[329] W. H. He, Y. X. Gao, F. Yuan, Y. N. Bao, F. Z. Liu, J. Q. Dong, Optimization of supercritical carbon dioxide extraction of gardenia fruit oil and the analysis of functional components, J. Am. Oil Chem. Soc., 87, 1071-1079 (2010).

[330] M. G. Bernardo-Gil, I. M. G. Lopes, M. Casquilho, M. A. Ribeiro, M. M. Esquivel, J. Empis, Supercritical carbon dioxide extraction of acorn oil, J. Supercrit. Fluids, 40, 344-348 (2007).

[331] K. W. Chan, M. Ismail, Supercritical carbon dioxide fluid extraction of Hibiscus cannabinus L. seed oil: A potential solvent-free and high antioxidative edible oil, Food Chem., 114, 970-975 (2009).

[332] A. A. Mariod, B. Matthaus, M. Ismail, Comparison of supercritical fluid and hexane extraction methods in extracting kenaf (Hibiscus cannabinus) seed oil lipids, J. Am. Oil Chem. Soc., 88, 931-935 (2011).

[333] S. Mazzutti, S. R. S. Ferreira, C. A. S. Riehl, A. Smania, F. A. Smania, J. Martinez, Supercritical fluid extraction of Agaricus brasiliensis: Antioxidant and antimicrobial activities, J. Supercrit. Fluids, 70, 48-56 (2012).

[334] M. G. Bernardo-Gil, M. Casquilho, M. M. Esquivel, M. A. Ribeiro, Supercritical fluid extraction of fig leaf gourd seeds oil: Fatty acids composition and extraction kinetics, J. Supercrit. Fluids, 49, 32-36 (2009).

[335] S. Jokić, Z. Zeković, S. Vidović, R. Sudar, I. Nemet, M. Bilić, D. Velić, Supercritical $\mathrm{CO}_{2}$ extraction of 
soybean oil: Process optimisation and triacylglycerol composition, Int. J. Food Sci. Technol., 45, 1939 1946 (2010).

[336] A. Rajaei, M. Barzegar, Y. Yamini, Supercritical fluid extraction of tea seed oil and its comparison with solvent extraction, Eur. Food Res. Technol., 220, 401-405 (2005).

[337] N. Mezzomo, B. R. Mileo, M. T. Friedrich, J. Martinez, S. R. S. Ferreira, Supercritical fluid extraction of peach (Prunus persica) almond oil: Process yield and extract composition, Bioresour. Technol., 101, 5622-5632 (2010).

[338] Y. Sanchez-Vicente, A. Cabanas, J. A. R. Renuncio, C. Pando, Supercritical fluid extraction of peach (Prunus persica) seed oil using carbon dioxide and ethanol, J. Supercrit. Fluids, 49, 167-173 (2009).

[339] G. Nimet, E. A. Da Silva, F. Palu, C. Dariva, L. D. Freitas, A. M. Neto, L. Cardozo, Extraction of sunflower (Heliantus annuus L.) oil with supercritical $\mathrm{CO}_{2}$ and subcritical propane: Experimental and modeling, Chem. Eng. J., 168, 262-268 (2011).

[340] S. M. Ghoreishi, E. Mardani, H. S. Ghaziaskar, Separation of gamma-linolenic and other polyunsaturated fatty acids from Boraginaceae via supercritical $\mathrm{CO}_{2}$, J. Sep. Sci., 34, 233-240 (2011).

[341] Q. A. Zhang, X. H. Fan, Z. Q. Zhang, B. S. Zhang, Z. Q. Zhang, X. Y. Jia, Optimization of $\mathrm{SC}-\mathrm{CO}_{2}$ extraction of oil from almond pretreated with autoclaving, Lwt-Food Sci. Technol., 42, 1530-1537 (2009).

[342] S. G. Ozkal, M. E. Yener, L. Bayindirli, Response surfaces of apricot kernel oil yield in supercritical carbon dioxide, Lwt-Food Sci. Technol., 38, 611-616 (2005).

[343] H. I. Castro-Vargas, L. I. Rodriguez-Varela, F. Parada-Alfonso, Guava (Psidium guajava L.) seed oil obtained with a homemade supercritical fluid extraction system using supercritical $\mathrm{CO}_{2}$ and co-solvent, $J$. Supercrit. Fluids, 56, 238-242 (2011).

[344] C. D. Andras, B. Simandi, F. Orsi, C. Lambrou, D. Missopolinou-Tatala, C. Panayiotou, J. Domokos, F. Doleschall, Supercritical carbon dioxide extraction of okra (Hibiscus esculentus L) seeds, J. Sci. Food Agric., 85, 1415-1419 (2005).

[345] [345]M. P. Corso, M. R. Fagundes-Klen, E. A. Silva, L. Cardozo, J. N. Santos, L. S. Freitas, C. Dariva, Extraction of sesame seed (Sesamun indicum L.) oil using compressed propane and supercritical carbon dioxide, J. Supercrit. Fluids, 52, 56-61 (2010).

[346] L. J. Wang, C. L. Weller, V. L. Schlegel, T. P. Carr, S. L. Cuppett, Comparison of supercritical $\mathrm{CO}_{2}$ and hexane extraction of lipids from Sorghum distill- ers grains, Eur. J. Lipid Sci. Technol., 109, 567-574 (2007).

[347] S. Zhang, Y. G. Zu, Y. J. Fu, M. Luo, W. Liu, J. Li, T. Efferth, Supercritical carbon dioxide extraction of seed oil from yellow horn (Xanthoceras sorbifolia Bunge.) and its anti-oxidant activity, Bioresour. Technol., 101, 2537-2544 (2010).

[348] W. Liu, Y. J. Fu, Y. G. Zu, M. H. Tong, N. Wu, X. L. Liu, S. Zhang, Supercritical carbon dioxide extraction of seed oil from Opuntia dillenii Haw. and its antioxidant activity, Food Chem., 114, 334-339 (2009).

[349] T. D. Weinhold, L. F. V. Bresciani, C. W. Tridapalli, R. A. Yunes, H. Hense, S. R. S. Ferreira, Polygala cyparissias oleoresin: Comparing $\mathrm{CO}_{2}$ and classical organic solvent extractions, Chem. Eng. Process., 47, 109-117 (2008).

[350] N. Hrabovski, S. Sinadinović-Fišer, B. Nikolovski, M. Sovilj, O. Borota, Phytosterols in pumpkin seed oil extracted by organic solvents and supercritical $\mathrm{CO}_{2}$, Eur. J. Lipid Sci. Technol., 114, 1204-1211 (2012).

[351] M. L. Martinez, M. A. Mattea, D. M. Maestri, Pressing and supercritical carbon dioxide extraction of walnut oil, J. Food Eng., 88, 399-404 (2008).

[352] L. D. S. Freitas, J. V. De Oliveira, C. Dariva, R. A. Jacques, E. B. Caramao, Extraction of grape seed oil using compressed carbon dioxide and propane: Extraction yields and characterization of free glycerol compounds, J. Agric. Food. Chem., 56, 2558-2564 (2008).

[353] S. K. Tang, C. R. Qin, H. G. Wang, S. F. Li, S. J. Tian, Study on supercritical extraction of lipids and enrichment of DHA from oil-rich microalgae, J. Supercrit. Fluids, 57, $44-49$ (2011).

[354] C. Da Porto, D. Decorti, F. Tubaro, Fatty acid composition and oxidation stability of hemp (Cannabis sativa L.) seed oil extracted by supercritical carbon dioxide, Ind. Crop. Prod., 36, 401-404 (2012).

[355] C. Da Porto, D. Voinovich, D. Decorti, A. Natolino, Response surface optimization of hemp seed (Cannabis sativa L.) oil yield and oxidation stability by supercritical carbon dioxide extraction, J. Supercrit. Fluids, 68, 45-51 (2012).

[356] A. M. A. Dias, P. Santos, I. J. Seabra, R. N. C. Junior, M. E. M. Braga, H. C. de Sousa, Spilanthol from Spilanthes acmella flowers, leaves and stems obtained by selective supercritical carbon dioxide extraction, J. Supercrit. Fluids, 61, 62-70 (2012).

[357] P. Bhattacharjee, D. Chatterjee, R. S. Singhal, Supercritical carbon dioxide extraction of squalene from Amaranthus paniculatus: Experiments and process 
characterization, Food Bioprocess Tech., 5, 25062521 (2012).

[358] L. Danielski, C. Zetzl, H. Hense, G. Brunner, A process line for the production of raffinated rice oil from rice bran, J. Supercrit. Fluids, 34, 133-141 (2005).

[359] W. H. Chen, C. H. Chen, C. M. J. Chang, B. C. Liau, D. Hsiang, Supercritical carbon dioxide extraction of triglycerides from Aquilaria crassna seeds, Sep. Purif. Technol., 73, 135-141 (2010).

[360] M. Markom, H. Singh, M. Hasan, Supercritical $\mathrm{CO}_{2}$ fractionation of crude palm oil, J. Supercrit. Fluids, 20, 45-53 (2001).

[361] W. H. Chen, C. H. Chen, C. M. J. Chang, Y. H. Chiu, D. Hsiang, Supercritical carbon dioxide extraction of triglycerides from Jatropha curcas L. seeds, J. Supercrit. Fluids, 51, 174-180 (2009).

[362] Y. J. Cheng, C. J. Shieh, Y. C. Wang, S. M. Lai, C. M. J. Chang, Supercritical carbon dioxide extraction of omega-3 oil compounds from Ficus awkeotsang makino achenes, Sep. Purif. Technol., 98, 62-68 (2012).

[363] M. M. R. de Melo, E. L. G. Oliveira, A. J. D. Silvestre, C. M. Silva, Supercritical fluid extraction of triterpenic acids from Eucalyptus globulus Bark, $J$. Supercrit. Fluids, 70, 137-145 (2012).

[364] R. M. A. Domingues, E. L. G. Oliveira, C. S. R. Freire, R. M. Couto, P. C. Simoes, C. P. Neto, A. J. D. Silvestre, C. M. Silva, Supercritical fluid extraction of Eucalyptus globulus Bark - A promising approach for triterpenoid production, Int. J. Mol. Sci., 13, 7648-7662 (2012).

[365] L. D. Freitas, R. A. Jacques, M. F. Richter, A. L. da Silva, E. B. Caramao, Pressurized liquid extraction of vitamin $\mathrm{E}$ from Brazilian grape seed oil, J. Chromatogr. A, 1200, 80-83 (2008).

[366] V. Y. Ixtaina, A. Vega, S. M. Nolasco, M. C. Tomas, M. Gimeno, E. Barzana, A. Tecante, Supercritical carbon dioxide extraction of oil from Mexican chia seed (Salvia hispanica L.): Characterization and process optimization, J. Supercrit. Fluids, 55, 192-199 (2010).

[367] J. A. R. Uribe, J. I. N. Perez, H. C. Kauil, G. R. Rubio, C. G. Alcocer, Extraction of oil from chia seeds with supercritical $\mathrm{CO}_{2}$, J. Supercrit. Fluids, 56, 174 178 (2011)

[368] L. Leo, L. Rescio, L. Ciurlia, G. Zacheo, Supercritical carbon dioxide extraction of oil and alpha-tocopherol from almond seeds, J. Sci. Food Agric., 85, 2167-2174 (2005).
[369] Y. Kawahito, M. Kondo, S. Machmudah, K. Sibano, M. Sasaki, M. Goto, Supercritical $\mathrm{CO}_{2}$ extraction of biological active compounds from loquat seed, Sep. Purif. Technol., 61, 130-135 (2008).

[370] A. Felfoldi-Gava, S. Szarka, B. Simandi, B. Blazics, B. Simon, A. Kery, Supercritical fluid extraction of Alnus glutinosa (L.) Gaertn, J. Supercrit. Fluids, 61, 55-61 (2012).

[371] M. R. Garcia-Risco, G. Vicente, G. Reglero, T. Fornari, Fractionation of thyme (Thymus vulgaris L.) by supercritical fluid extraction and chromatography, $J$. Supercrit. Fluids, 55, 949-954 (2011).

[372] B. Kinsella, J. O’Mahony, E. Malone, M. Moloney, H. Cantwell, A. Furey, M. Danaher, Current trends in sample preparation for growth promoter and veterinary drug residue analysis, J. Chromatogr. A, 1216, 7977-8015 (2009).

[373] D. M. Pavlović, S. Babić, A. J. M. Horvat, M. Kastelan-Macan, Sample preparation in analysis of pharmaceuticals, Trac-Trend. Anal. Chem., 26, 1062-1075 (2007).

[374] A. B. A. de Azevedo, P. Mazzafera, R. S. Mohamed, S. de Melo, T. G. Kieckbusch, Extraction of caffeine, chlorogenic acids and lipids from green coffee beans using supercritical carbon dioxide and co-solvents, Braz. J. Chem. Eng., 25, 543-552 (2008).

[375] R. A. Jacques, J. G. Santos, C. Dariva, J. V. Oliveira, E. B. Caramao, GC/MS characterization of mate tea leaves extracts obtained from high-pressure $\mathrm{CO}_{2}$ extraction, J. Supercrit. Fluids, 40, 354-359 (2007).

[376] W. J. Kim, J. D. Kim, J. Kim, S. G. Oh, Y. W. Lee, Selective caffeine removal from green tea using supercritical carbon dioxide extraction, J. Food Eng., 89, 303-309 (2008).

[377] W. J. Kim, J. D. Kim, S. G. Oh, Supercritical carbon dioxide extraction of caffeine from Korean green tea, Sep. Sci. Technol., 42, 3229-3242 (2007).

[378] S. M. Lee, H. S. Lee, K. H. Kim, K. O. Kim, Sensory characteristics and consumer acceptability of decaffeinated green teas, J. Food Sci., 74, S135-S141 (2009).

[379] J. Tello, M. Viguera, L. Calvo, Extraction of caffeine from robusta coffee (Coffea canephora var. robusta) Husks using supercritical carbon dioxide, J. Supercrit. Fluids, 59, 53-60 (2011).

[380] B. Liu, H. L. Jiang, B. Shen, Y. L. Chang, Supercritical fluid extraction of sinomenine from Sinomenium acutum (Thumb) Rehd et Wils, J. Chromatogr. A, 1075, 213-215 (2005). 
[381] D. C. Soares, C. G. Pereira, M. A. A. Meireles, E. M. Saraiva, Leishmanicidal activity of a supercritical fluid fraction obtained from Tabernaemontana catharinensis, Parasitol. Int., 56, 135-139 (2007).

[382] A. Verma, K. Hartonen, M. L. Riekkola, Optimisation of supercritical fluid extraction of indole alkaloids from Catharanthus roseus using experimental design methodology - comparison with other extraction techniques, Phytochem. Anal, 19, 52-63 (2008).

[383] E. L. Cardozo, L. Cardozo, O. Ferrarese, E. F. Zanoelo, Selective liquid $\mathrm{CO}_{2}$ extraction of purine alkaloids in different ilex paraguariensis progenies grown under environmental influences, J. Agric. Food. Chem., 55, 6835-6841 (2007).

[384] B. Liu, B. Shen, F. Guo, Y. L. Chang, Optimization of supercritical fluid extraction of dl-tetrahydropalmatine from rhizome of Corydalis yanhusuo wt wang with orthogonal array design, Sep. Purif. Technol., 64, 242-246 (2008).

[385] N. S. Karbalaie, C. Ghotbi, V. Taghikhani, Y. Yamini, Experimental study and modeling of supercritical extraction of nicotine from tabacco leaves, Iranian Journal of Chemistry \& Chemical Engineering - International English Edition, 28, 51-59 (2009).
[386] J. A. Xiao, B. Q. Tian, B. J. Xie, E. N. Yang, J. Shi, Z. D. Sun, Supercritical fluid extraction and identification of isoquinoline alkaloids from leaves of Nelumbo nucifera Gaertn, Eur. Food Res. Technol., 231, 407-414 (2010).

[387] M. Zougagh, A. Rios, M. Valcarcel, Determination of total safranal by in situ acid hydrolysis in supercritical fluid media: Application to the quality control of commercial saffron, Anal. Chim. Acta, 578, 117-121, (2006).

[388] B. Liu, F. Guo, Y. L. Chang, H. L. Jiang, Q. A. Wang, Optimization of extraction of evodiamine and rutaecarpine from fruit of Evodia rutaecarpa using modified supercritical $\mathrm{CO}_{2}$, J. Chromatogr. A, 1217, 7833-7839, (2010).

[389] S. Glišić, A. Smelcerović, S. Zuehlke, M. Spiteller, D. Skala, Extraction of hyperforin and adhyperforin from st. John's wort (Hypericum perforatum L.) by supercritical carbon dioxide, J. Supercrit. Fluids, 45, 332-337 (2008).

[390] A. Ruiz-Rodriguez, M. R. Bronze, M. N. da Ponte, Supercritical fluid extraction of tobacco leaves: A preliminary study on the extraction of solanesol, $J$. Supercrit. Fluids, 45, 171-176 (2008). 